\title{
Toward a unified interpretation of quark and lepton mixing from flavor and CP symmetries
}

\section{Cai-Chang Li, Jun-Nan Lu and Gui-Jun Ding}

University of Science and Technology of China,

No. 96, JinZhai Road, Baohe District, Hefei, Anhui, 230026 China

E-mail: lcc0915@mail.ustc.edu.cn, hitman@mail.ustc.edu.cn,

dinggj@ustc.edu.cn

ABSTRACT: We discussed the scenario that a discrete flavor group combined with $\mathrm{CP}$ symmetry is broken to $Z_{2} \times C P$ in both neutrino and charged lepton sectors. All lepton mixing angles and $\mathrm{CP}$ violation phases are predicted to depend on two free parameters $\theta_{l}$ and $\theta_{\nu}$ varying in the range of $[0, \pi)$. As an example, we comprehensively study the lepton mixing patterns which can be derived from the flavor group $\Delta\left(6 n^{2}\right)$ and CP symmetry. Three kinds of phenomenologically viable lepton mixing matrices are obtained up to row and column permutations. We further extend this approach to the quark sector. The precisely measured quark mixing angles and CP invariant can be accommodated for certain values of the free parameters $\theta_{u}$ and $\theta_{d}$. A simultaneous description of quark and lepton flavor mixing structures can be achieved from a common flavor group $\Delta\left(6 n^{2}\right)$ and $\mathrm{CP}$, and accordingly the smallest value of the group index $n$ is $n=7$.

Keywords: CP violation, Discrete Symmetries, Neutrino Physics

ARXIV EPRINT: 1706.04576 


\section{Contents}

1 Introduction 1

2 Framework 3

3 Lepton mixing patterns from $\Delta\left(6 n^{2}\right)$ and CP symmetries $\quad 7$

4 Quark mixing from $\Delta\left(6 n^{2}\right)$ and CP symmetries 33

5 Summary and conclusions $\quad 41$

\section{Introduction}

It is well-known that the flavor mixings in the quark and lepton sectors are completely different [1]. All the three quark mixing angles are small with the Cabibbo angle $\theta_{\mathrm{C}} \simeq 13^{\circ}$ being the largest, while in the lepton sector both solar and atmospheric mixing angles are large and the reactor angle is of the same order as the Cabibbo angle. As regards the $\mathrm{CP}$ violation, it is well established that the description of $\mathrm{CP}$ violation in terms of the Kobayashi-Maskawa mechanism [2] agrees with all measurements to date [1], and the CP violation phase has been precisely measured. The analogous mixing matrix for leptons has three CP-violating phases: one Dirac CP phase $\delta_{\mathrm{CP}}$ and two Majorana CP phases $\alpha_{21}$ and $\alpha_{31}$ if neutrinos are Majorana particles. The values of these three leptonic $\mathrm{CP}$ violation phases are unknown although there is some as yet inconclusive evidence for $\delta_{\mathrm{CP}}$ around $3 \pi / 2$ [3-6]. The global fits of the current neutrino oscillation data do not allow to pin down a preferred value of $\delta_{\mathrm{CP}}$ at the $3 \sigma$ confidence level [7-9].

Understanding the origin of the quark and lepton flavor mixing patterns is a fundamental problem in particle physics. The special structure of the lepton mixing matrix provides a strong hint for a flavor symmetry which is broken in a non-trivial way. The nonabelian discrete flavor symmetry has been widely exploited to explain the fermion mass hierarchies and flavor mixing puzzles (for reviews see e.g. [10-14]). In this approach, it is generally assumed that the theory possesses a flavor symmetry at certain high energy scale, which is broken to different residual subgroups in the charged lepton and neutrino sectors at lower energies. The mismatch between the two residual subgroups allows one to predict the lepton mixing matrix while the Majorana phases are not constrained. If the residual symmetries of the neutrino and charged lepton mass matrices wholly belong to the postulated parent flavor symmetry, the mixing patterns which can be derived from finite discrete groups are quite restricted, the second column of the lepton mixing matrix is $(1,1,1)^{T} / \sqrt{3}$ in order to be compatible with experimental data, and the Dirac CP phase is either 0 or $\pi$ [15-19]. If the residual symmetries of the neutrino and charged lepton mass 
terms partially belong to the parent flavor symmetry group, one column or one row of the mixing matrix can be fixed such that some correlations between neutrino mixing angles and Dirac CP phase can be predicted [20-23]. The paradigm of discrete flavor symmetry has also been used to explain quark mixing [19, 24-29]. It is found that only the Cabibbo mixing between the first two generations of quarks can be generated, no matter whether the left-handed quarks are assigned to an irreducible triplet representation of the flavor group, or to a reducible triplet which can decompose into a two-dimensional and a onedimensional representation [19, 29]. For example, a phenomenologically acceptable value of $\theta_{\mathrm{C}}=\pi / 14$ can be naturally obtained from the simple dihedral group $D_{14}$ [19, 24, 25]. Since the dihedral group $D_{14}$ is a subgroup of $\Delta\left(6 n^{2}\right)$ with $n=7$, this result can be easily incorporated in a study of the group $\Delta(294)$.

The flavor symmetry is extended to involve also CP as symmetry in recent years since generic neutrino and charged lepton mass matrices admit residual CP symmetry besides residual flavor symmetry [30-34]. The CP transformation acts on the flavor space in a non-trivial way. As a result, the CP symmetry should be consistently implemented in a theory based on discrete flavor symmetry and certain consistency condition has to be satisfied [30, 35-37]. Discrete flavor symmetry combined with CP symmetry is a rather predictive framework, and one can determine all the lepton mixing angles and CP phases in terms of few free parameters [30,38-63]. In the charged lepton diagonal basis, the residual CP transformation can be classified according to the number of zero entries [64, 65]. Moreover, small discrete groups such as $A_{4}$ [38-40] and $S_{4}$ [30, 41-45] can already accommodate the experimental data on lepton mixing angles and predict maximal Dirac phase. Other non-regular values of $\delta_{\mathrm{CP}}$ which is neither trivial nor maximal can be obtained from larger flavor symmetry groups [46-51, 54-57, 59]. Furthermore, the combination of flavor and CP symmetries can also restrict the high energy CP phases that are relevant for the baryon asymmetry of the Universe in both the flavored and unflavored leptogenesis [59, 66-68]. In the most widely discussed scenarios involving CP, it is usually assumed that the original flavor and $\mathrm{CP}$ symmetries are broken to an abelian subgroup in the charged lepton sector and to $Z_{2} \times C P$ in the neutrino sector [30, 38-51, 54-59], consequently the lepton mixing matrix is predicted to contain only one free real parameter $\theta$. Although this approach can successfully explain the measured lepton mixing angles and predict $\mathrm{CP}$ violation phases, it is not possible to derive the hierarchical mixing pattern among quarks in a similar way. ${ }^{1}$

Other possible schemes to predict lepton flavor mixing from discrete flavor symmetry and CP symmetry have been investigated in the literature [60-63]. The scenario that the residual symmetry of both the neutrino and the charged lepton sector is $Z_{2} \times C P$ is considered in refs. [60,61], and the resulting lepton mixing angles as well as all CP phases in this scheme depend on two free real parameters $\theta_{\nu}$ and $\theta_{l}$. The authors of $[62,63]$ consider a second scenario where the residual symmetry is $Z_{2}$ in the charged lepton and

\footnotetext{
${ }^{1}$ As for the lepton, if the flavor and $\mathrm{CP}$ symmetries are broken to an abelian subgroup and $Z_{2} \times C P$ in the up quark and down quark sectors, the CKM mixing matrix would be predicted to take similar form as the lepton mixing matrix in the semidirect approach, the small quark mixing angles and the precisely measured quark CP violation phases can not be explained simultaneously.
} 
$Z_{2} \times C P$ in the neutrino sector, all the lepton mixing angles and the CP phases are functions of three free parameters. In the present paper, we perform a comprehensive analysis of lepton mixing patterns which arise from the breaking of $\Delta\left(6 n^{2}\right)$ flavor group and CP to distinct residual subgroups $Z_{2} \times C P$ in the neutrino and charged lepton sectors. In the same fashion, we find that the experimentally measured values of quark mixing angles and $\mathrm{CP}$ violation phase can be accommodated if the residual symmetry of both the up- and down-type quarks mass matrices is $Z_{2} \times C P$. The resulting CKM mixing matrix depends on two free parameters $\theta_{u}$ and $\theta_{d}$. It is notable that a simultaneous description of quark and lepton mixing can be achieved in a common flavor symmetry group such as $\Delta(294)$ combined with CP symmetry. In our working basis, the CP transformation compatible with flavor symmetry $\Delta(294)$ is of the same form as the flavor group transformation.

The structure of this paper is as follows: in section 2 we present the master formula for the lepton mixing matrix when a general flavor symmetry combined with CP symmetry is broken down to $Z_{2} \times C P$ in both neutrino and charged lepton sectors. The prediction for the quark CKM mixing matrix is also presented in the case that a residual symmetry $Z_{2} \times C P$ is preserved by the up and down quark mass matrices. In section 3 we perform a detailed study for the flavor group $\Delta\left(6 n^{2}\right)$ combined with CP symmetry. All possible residual symmetries of the structure $Z_{2} \times C P$ are considered and we present the resulting analytic expressions for the lepton mixing angles and $\mathrm{CP}$ invariants. In each case we also perform a numerical analysis for small values of the group index $n$ which can admit reasonable agreement with experimental data. Our analysis is extended to the quark sector in section 4. Only one type of combination of residual symmetries is capable of describing the hierarchical quark mixing angles together with the precisely measured quark CP violation phase. Moreover phenomenologically viable quark and lepton mixing patterns can be simultaneously obtained from certain $\Delta\left(6 n^{2}\right)$ flavor group combined with CP symmetry. The different quark and lepton flavor mixing structures arise from different underlying residual symmetries in this approach. Finally we conclude in section 5 .

\section{Framework}

In the paradigm of discrete flavor symmetry combined with generalized CP symmetry, the original flavor and CP symmetries are generically assumed to be broken down to $Z_{2} \times C P$ in the neutrino sector and an abelian subgroup in the charged lepton sector. In this work, we shall investigate the scenario that the remnant symmetry of both the neutrino and charged lepton mass matrices is $Z_{2} \times C P$. The master formula for the lepton mixing matrix would be derived in the following. In this approach, the non-trivial lepton mixing matrix arises from the misalignment between the two residual symmetries of the neutrino and charged lepton sectors, and one doesn't need to consider the underlying mechanism to dynamically achieve the assumed residual symmetry. Furthermore, we shall extend this approach to the quark sector. As usual we assign the three generation of the left-handed lepton fields to an irreducible three dimensional representation $\mathbf{3}$ of the flavor symmetry group.

We denote the remnant symmetries of the neutrino and charged lepton mass matrices as $Z_{2}^{g_{\nu}} \times X_{\nu}$ and $Z_{2}^{g_{l}} \times X_{l}$ respectively, where $g_{\nu}$ and $g_{l}$ refer to the generators of the $Z_{2}$ 
residual flavor symmetry groups with $g_{\nu}^{2}=g_{l}^{2}=1$. The remnant CP transformations $X_{\nu}$ and $X_{l}$ are $3 \times 3$ unitary and symmetric matrices. These residual symmetries are well defined if and only if the following consistency conditions are satisfied [30-33],

$$
X_{l} \rho_{\mathbf{3}}^{*}\left(g_{l}\right) X_{l}^{-1}=\rho_{\mathbf{3}}\left(g_{l}\right), \quad X_{\nu} \rho_{\mathbf{3}}^{*}\left(g_{\nu}\right) X_{\nu}^{-1}=\rho_{\mathbf{3}}\left(g_{\nu}\right),
$$

where $\rho_{\mathbf{3}}\left(g_{l}\right)$ and $\rho_{\mathbf{3}}\left(g_{\nu}\right)$ denote the representation matrices of the elements $g_{l}$ and $g_{\nu}$ in the three dimensional representation 3. The remnant symmetries $Z_{2}^{g_{\nu}} \times X_{\nu}$ in neutrino sector and $Z_{2}^{g_{l}} \times X_{l}$ in charged lepton sector imply that the charged mass matrix $m_{l}$ and the neutrino mass matrix $m_{\nu}$ should fulfill

$$
\begin{aligned}
\rho_{\mathbf{3}}^{\dagger}\left(g_{l}\right) m_{l}^{\dagger} m_{l} \rho_{\mathbf{3}}\left(g_{l}\right) & =m_{l}^{\dagger} m_{l}, & X_{l}^{\dagger} m_{l}^{\dagger} m_{l} X_{l} & =\left(m_{l}^{\dagger} m_{l}\right)^{*}, \\
\rho_{\mathbf{3}}^{T}\left(g_{\nu}\right) m_{\nu} \rho_{\mathbf{3}}\left(g_{\nu}\right) & =m_{\nu}, & X_{\nu}^{T} m_{\nu} X_{\nu} & =m_{\nu}^{*},
\end{aligned}
$$

where the charged lepton mass matrix $m_{l}$ is defined in the right-left basis $\bar{l}_{R} m_{l} l_{L}$. Once the explicit form of the residual symmetries are given, the charged lepton mass matrix $m_{l}^{\dagger} m_{l}$ and the neutrino mass matrix $m_{\nu}$ can be reconstructed straightforwardly from eqs. (2.2a), (2.2b), and subsequently the PMNS mixing matrix can be determined by diagonalizing $m_{l}^{\dagger} m_{l}$ and $m_{\nu}$. In fact one can also fix the mixing matrix without resorting to the mass matrices.

Firstly we start from the charged lepton sector. The transformation of the lefthanded charged leptons used to diagonalize $m_{l}$ is denoted as $U_{l}$, i.e., $U_{l}^{\dagger} m_{l}^{\dagger} m_{l} U_{l}=$ $\operatorname{diag}\left(m_{e}^{2}, m_{\mu}^{2}, m_{\tau}^{2}\right)$, then from eq. (2.2a) we find that the residual symmetry $Z_{2}^{g_{l}} \times X_{l}$ leads to the following constraints on the unitary transformation $U_{l}$,

$$
\begin{aligned}
U_{l}^{\dagger} \rho_{3}\left(g_{l}\right) U_{l} & =\operatorname{diag}( \pm 1, \pm 1, \pm 1), \\
U_{l}^{\dagger} X_{l} U_{l}^{*} & =\operatorname{diag}\left(e^{i \alpha_{e}}, e^{i \alpha_{\mu}}, e^{i \alpha_{\tau}}\right) \equiv Q_{l}^{2},
\end{aligned}
$$

where $\alpha_{e, \mu, \tau}$ are arbitrary real parameters. Obviously eq. (2.4) implies that the residual CP transformation $X_{l}$ is a symmetric unitary matrix. Since the element $g_{l}$ is of order 2 , each eigenvalue of $\rho_{\mathbf{3}}\left(g_{l}\right)$ is either +1 or -1 . That is exactly the reason why the diagonal entries on the right-handed side of eq. (2.3) are \pm 1 . Without loss of generality, we take the three eigenvalues of $\rho_{\mathbf{3}}\left(g_{l}\right)$ to be $+1,-1$ and -1 . Hence eq. (2.3) can be written as

$$
U_{l}^{\dagger} \rho_{3}\left(g_{l}\right) U_{l}=P_{l}^{T} \operatorname{diag}(1,-1,-1) P_{l},
$$

where $P_{l}$ is a generic three dimensional permutation matrix. Since the residual CP transformation $X_{l}$ must be a unitary and symmetric matrix to avoid partially degenerate charged lepton masses, after performing the Takagi factorization it can be written as

$$
X_{l}=\Sigma_{l} \Sigma_{l}^{T}
$$

where $\Sigma_{l}$ is a diagonalization matrix of $\rho_{\mathbf{3}}\left(g_{l}\right)$ with [59]

$$
\Sigma_{l}^{\dagger} \rho_{\mathbf{3}}\left(g_{l}\right) \Sigma_{l}=\operatorname{diag}(1,-1,-1) .
$$


The remnant symmetry $Z_{2}^{g_{l}} \times X_{l}$ of the charged lepton sector enforces the unitary transformation $U_{l}$ to be of the following form [59]

$$
U_{l}=\Sigma_{l} S_{23}\left(\theta_{l}\right) P_{l} Q_{l}^{\dagger}
$$

with

$$
S_{23}\left(\theta_{l}\right)=\left(\begin{array}{ccc}
1 & 0 & 0 \\
0 & \cos \theta_{l} & \sin \theta_{l} \\
0 & -\sin \theta_{l} & \cos \theta_{l}
\end{array}\right),
$$

where $\theta_{l}$ is a real parameter in the fundamental interval $[0, \pi)$. The analyses of the residual symmetry $Z_{2}^{g_{\nu}} \times X_{\nu}$ and the resulting constraints on the unitary transformation $U_{\nu}$ which diagonalizes the neutrino mass matrix as $U_{\nu}^{T} m_{\nu} U_{\nu}=\operatorname{diag}\left(m_{1}, m_{2}, m_{3}\right)$ can be found in ref. [59]. The Takagi factorization matrix $\Sigma_{\nu}$ for $X_{\nu}$ has the properties

$$
X_{\nu}=\Sigma_{\nu} \Sigma_{\nu}^{T}, \quad \Sigma_{\nu}^{\dagger} \rho_{\mathbf{3}}\left(g_{\nu}\right) \Sigma_{\nu}= \pm \operatorname{diag}(1,-1,-1) .
$$

Then the neutrino matrix fulfilling the residual symmetry invariant condition of eq. $(2.2 \mathrm{~b})$ can be diagonalized by the following unitary matrix $U_{\nu}$,

$$
U_{\nu}=\Sigma_{\nu} S_{23}\left(\theta_{\nu}\right) P_{\nu} Q_{\nu}^{\dagger},
$$

where the free rotation angle $\theta_{\nu}$ is in the range of $0 \leq \theta_{\nu}<\pi$, and $P_{\nu}$ is a permutation matrix. The unitary matrix $Q_{\nu}$ is diagonal with entries \pm 1 and $\pm i$, it is necessary to making neutrino masses non-negative. As a result, the assumed residual symmetry allows us to pin down the lepton mixing matrix as

$$
U \equiv U_{l}^{\dagger} U_{\nu}=Q_{l} P_{l}^{T} S_{23}^{T}\left(\theta_{l}\right) \Sigma S_{23}\left(\theta_{\nu}\right) P_{\nu} Q_{\nu}^{\dagger}
$$

with

$$
\Sigma \equiv \Sigma_{l}^{\dagger} \Sigma_{\nu}
$$

It is remarkable that one element of the PMNS matrix is fixed to be certain constant by residual symmetry in this approach, and the fixed element is the (11) entry of $\Sigma$. The phase matrix $Q_{l}$ can be absorbed by the charged lepton fields and the effect of $Q_{\nu}$ is a possible change of the Majorana phases by $\pi$. Moreover, we see that the mixing matrix as well mixing angles and CP phases are predicted to depend on only two free real parameters $\theta_{l}$ and $\theta_{\nu}$. In addition, the fundamental interval of both $\theta_{l}$ and $\theta_{\nu}$ is $[0, \pi)$, the reason is because the lepton mixing matrix $U$ in eq. (2.12) fulfills

$$
\begin{aligned}
& U\left(\theta_{l}+\pi, \theta_{\nu}\right)=P_{l}^{T} \operatorname{diag}(1,-1,-1) P_{l} U\left(\theta_{l}, \theta_{\nu}\right), \\
& U\left(\theta_{l}, \theta_{\nu}+\pi\right)=U\left(\theta_{l}, \theta_{\nu}\right) P_{\nu}^{T} \operatorname{diag}(1,-1,-1) P_{\nu},
\end{aligned}
$$

where the diagonal matrices $P_{l}^{T} \operatorname{diag}(1,-1,-1) P_{l}$ and $P_{\nu}^{T} \operatorname{diag}(1,-1,-1) P_{\nu}$ can be absorbed into $Q_{l}$ and $Q_{\nu}$, respectively. Because both the charged lepton and neutrino masses can not be predicted in this model independent approach, the PMNS matrix is determined up to permutations of rows and columns, and consequently $U$ is multiplied by $P_{l}^{T}$ and $P_{\nu}$ 
from the left-hand side and the right-hand side respectively. The permutation matrices $P_{l}$ and $P_{\nu}$ can take six possible values and they can be generated from

$$
P_{12}=\left(\begin{array}{lll}
0 & 1 & 0 \\
1 & 0 & 0 \\
0 & 0 & 1
\end{array}\right), \quad P_{13}=\left(\begin{array}{lll}
0 & 0 & 1 \\
0 & 1 & 0 \\
1 & 0 & 0
\end{array}\right), \quad P_{23}=\left(\begin{array}{lll}
1 & 0 & 0 \\
0 & 0 & 1 \\
0 & 1 & 0
\end{array}\right) .
$$

Furthermore the lepton mixing matrix $U$ has the following symmetry properties,

$$
P_{l}^{T} P_{23} P_{l} U\left(\theta_{l}, \theta_{\nu}\right)=Q_{l}^{\prime} U\left(\theta_{l}+\frac{\pi}{2}, \theta_{\nu}\right), \quad U\left(\theta_{l}, \theta_{\nu}\right) P_{\nu}^{T} P_{23} P_{\nu}=U\left(\theta_{l}, \theta_{\nu}+\frac{\pi}{2}\right) Q_{\nu}^{\prime},
$$

where $Q_{l}^{\prime}$ and $Q_{\nu}^{\prime}$ are given by

$$
Q_{l}^{\prime}=P_{l}^{T} P_{23} P_{l} Q_{l} P_{l}^{T} P_{23}^{T} \operatorname{diag}(1,-1,1) P_{l} Q_{l}^{\dagger}, \quad Q_{\nu}^{\prime}=Q_{\nu} P_{\nu}^{T} \operatorname{diag}(1,-1,1) P_{23}^{T} P_{\nu} Q_{\nu}^{\dagger} P_{\nu}^{T} P_{23} P_{\nu} .
$$

It is easy to check that $Q_{l}^{\prime}$ is an arbitrary phase matrix, and $Q_{\nu}^{\prime}$ is diagonal with elements equal to \pm 1 and $\pm i$. The contributions of $Q_{l}^{\prime}$ and $Q_{\nu}^{\prime}$ can be absorbed into $Q_{l}$ and $Q_{\nu}$ respectively. Therefore eq. (2.16) indicates that the row permutation $P_{l}^{T} P_{23} P_{l}$ and column permutation $P_{\nu}^{T} P_{23} P_{\nu}$ of the PMNS matrix $U$ doesn't give rise to new mixing pattern for any given values of $P_{l}$ and $P_{\nu}$. As a consequence, only nine independent mixing patterns can be obtained out of the 36 possible permutations of rows and columns. Accordingly the element completely fixed by residual symmetry can be in any of the nine positions of the mixing matrix.

If the roles of $Z^{g_{l}} \times X_{l}$ and $Z^{g_{\nu}} \times X_{\nu}$ are exchanged, the lepton mixing matrix $U$ in eq. (2.12) would transform into its hermitian conjugate. Moreover, if a pair of residual subgroups $\left\{Z^{g_{l}^{\prime}} \times X_{l}^{\prime}, Z^{g_{\nu}^{\prime}} \times X_{\nu}^{\prime}\right\}$ are related to $\left\{Z^{g_{l}} \times X_{l}, Z^{g_{\nu}} \times X_{\nu}\right\}$ by a similarity transformation,

$$
\begin{aligned}
\rho_{\mathbf{3}}\left(g_{l}^{\prime}\right) & =\Omega \rho_{\mathbf{3}}\left(g_{l}\right) \Omega^{-1}, & \rho_{\mathbf{3}}\left(g_{\nu}^{\prime}\right) & =\Omega \rho_{\mathbf{3}}\left(g_{\nu}\right) \Omega^{-1}, \\
X_{l}^{\prime} & =\Omega X_{l} \Omega^{T}, & X_{\nu}^{\prime} & =\Omega X_{\nu} \Omega^{T},
\end{aligned}
$$

where $\Omega$ is a unitary matrix, both residual symmetries would lead to the same result for the PMNS mixing matrix. The reason is because if $\Sigma_{l}$ and $\Sigma_{\nu}$ are the Takagi factorization matrices of $X_{l}$ and $X_{\nu}$ respectively, and they diagonalize $\rho_{\mathbf{3}}\left(g_{l}\right)$ and $\rho_{\mathbf{3}}\left(g_{\nu}\right)$, the desired Takagi factorization of $X_{l}^{\prime}$ and $X_{\nu}^{\prime}$ would be $\Omega \Sigma_{l}$ and $\Omega \Sigma_{\nu}$ respectively. Using the master formula of eq. (2.12) we would obtain the same lepton mixing matrix.

We can extend this approach to the quark sector to derive the quark flavor mixing in a similar way. The residual symmetries of the up type quark and down type quark mass matrices are assumed to be $Z_{2}^{g_{u}} \times X_{u}$ and $Z_{2}^{g_{d}} \times X_{d}$ respectively with $g_{u}^{2}=g_{d}^{2}=1$. Similar to the left-handed leptons, the three left-handed quarks are assigned to an irreducible triplet $\mathbf{3}$ of the flavor symmetry group. The observed strong quark mass hierarchies are expected to be explained in a concrete model by employing auxiliary symmetry either discrete like $Z_{m}$ or continuous like $\mathrm{U}(1)$, under which the right-handed quark fields transform differently. The residual flavor and CP symmetries have to fulfill the following consistency conditions,

$$
X_{u} \rho_{\mathbf{3}}^{*}\left(g_{u}\right) X_{u}^{-1}=\rho_{\mathbf{3}}\left(g_{u}\right), \quad X_{d} \rho_{\mathbf{3}}^{*}\left(g_{d}\right) X_{d}^{-1}=\rho_{\mathbf{3}}\left(g_{d}\right) .
$$


For the residual symmetries to hold, the hermitian combinations $m_{U}^{\dagger} m_{U}$ and $m_{D}^{\dagger} m_{D}$ should be invariant under the action of the residual subgroups, i.e.

$$
\begin{array}{rlrl}
\rho_{\mathbf{3}}^{\dagger}\left(g_{u}\right) m_{U}^{\dagger} m_{U} \rho_{\mathbf{3}}\left(g_{u}\right) & =m_{U}^{\dagger} m_{U}, & & X_{u}^{\dagger} m_{U}^{\dagger} m_{U} X_{u}=\left(m_{U}^{\dagger} m_{U}\right)^{*}, \\
\rho_{\mathbf{3}}^{\dagger}\left(g_{d}\right) m_{D}^{\dagger} m_{D} \rho_{\mathbf{3}}\left(g_{d}\right)=m_{D}^{\dagger} m_{D}, & X_{d}^{\dagger} m_{D}^{\dagger} m_{D} X_{d}=\left(m_{D}^{\dagger} m_{D}\right)^{*},
\end{array}
$$

where $m_{U}$ and $m_{D}$ denote the up quark and down quark mass matrices respectively. Similar to the lepton sector, the constraints in eq. (2.20) can be conveniently solved by finding the appropriate Takagi factorization matrices for the residual CP transformations $\Sigma_{u}$ and $\Sigma_{d}$ with the properties

$$
\begin{array}{ll}
X_{u}=\Sigma_{u} \Sigma_{u}^{T}, & \Sigma_{u}^{\dagger} \rho_{\mathbf{3}}\left(g_{u}\right) \Sigma_{u}= \pm \operatorname{diag}(1,-1,-1), \\
X_{d}=\Sigma_{d} \Sigma_{d}^{T}, & \Sigma_{d}^{\dagger} \rho_{\mathbf{3}}\left(g_{d}\right) \Sigma_{d}= \pm \operatorname{diag}(1,-1,-1) .
\end{array}
$$

Then the unitary transformations $U_{u}$ and $U_{d}$ which diagonalize $m_{U}^{\dagger} m_{U}$ and $m_{D}^{\dagger} m_{D}$ respectively would take the form

$$
U_{u}=\Sigma_{u} S_{23}\left(\theta_{u}\right) P_{u} Q_{u}^{\dagger}, \quad U_{d}=\Sigma_{d} S_{23}\left(\theta_{d}\right) P_{d} Q_{d}^{\dagger} .
$$

As a result, the CKM mixing matrix $V$ is determined to be

$$
V=U_{u}^{\dagger} U_{d}=Q_{u} P_{u}^{T} S_{23}^{T}\left(\theta_{u}\right) \Sigma_{u}^{\dagger} \Sigma_{d} S_{23}\left(\theta_{d}\right) P_{d} Q_{d}^{\dagger},
$$

where the rotation angles $\theta_{u}$ and $\theta_{d}$ are in the fundamental interval of $[0, \pi), Q_{u}$ and $Q_{d}$ are arbitrary diagonal phase matrices and they can be absorbed by the quark fields. In addition, $P_{u}$ and $P_{d}$ are generic three dimensional permutation matrices since the order of the up type quark and down type quark masses is not constrained in this approach. Similar to the lepton sector, we see that one element of the CKM mixing matrix is fixed by the residual symmetry. The three quark mixing angles and the $\mathrm{CP}$ phase are determined in terms of only two free parameters $\theta_{u}$ and $\theta_{d}$ which can take values between 0 and $\pi$.

\section{Lepton mixing patterns from $\Delta\left(6 n^{2}\right)$ and CP symmetries}

In this section, as a concrete example, we shall perform a comprehensive analyze of the lepton mixing patterns arising from the $\Delta\left(6 n^{2}\right)[69,70]$ flavor group and CP symmetries which are broken down to $Z_{2} \times C P$ in the neutrino and charged lepton sectors. All possible admissible residual subgroups of the structure $Z_{2} \times C P$ would be considered, and the phenomenological predictions for lepton mixing matrix as well as neutrinoless double decay would be discussed.

$\Delta\left(6 n^{2}\right)$ and its subgroups have been widely exploited as flavor symmetry to constrain the lepton flavor mixing in the literature $[16,54-56] . \Delta\left(6 n^{2}\right)$ group can be conveniently generated by four generators $a, b, c$ and $d$, and the multiplication rules are [56, 70]

$$
\begin{aligned}
a^{3} & =b^{2}=(a b)^{2}=c^{n}=d^{n}=1, \quad c d=d c, \\
a c a^{-1} & =c^{-1} d^{-1}, \quad a d a^{-1}=c, \quad b c b^{-1}=d^{-1}, \quad b d b^{-1}=c^{-1} .
\end{aligned}
$$


All the conjugacy classes, inequivalent irreducible representations and Clebsch-Gordan coefficients of $\Delta\left(6 n^{2}\right)$ group have been presented in ref. [56]. The $\Delta\left(6 n^{2}\right)$ group has $2(n-1)$ three-dimensional irreducible representations denoted by $\mathbf{3}_{k}^{l}$ and explicitly generated by [56]:

$$
a=\left(\begin{array}{lll}
0 & 1 & 0 \\
0 & 0 & 1 \\
1 & 0 & 0
\end{array}\right), \quad b=(-)^{k+1}\left(\begin{array}{ccc}
0 & 0 & 1 \\
0 & 1 & 0 \\
1 & 0 & 0
\end{array}\right), \quad c=\left(\begin{array}{ccc}
\eta^{l} & 0 & 0 \\
0 & \eta^{-l} & 0 \\
0 & 0 & 1
\end{array}\right), \quad d=\left(\begin{array}{ccc}
1 & 0 & 0 \\
0 & \eta^{l} & 0 \\
0 & 0 & \eta^{-l}
\end{array}\right)
$$

where $\eta=e^{2 \pi i / n}, k=1,2$ and $l=1,2, \ldots, n-1$. We shall restrict ourselves to working with only faithful irreducible representations of $\Delta\left(6 n^{2}\right)$. Thus we exclude all triplet representations where $l$ divides $n$, since they are unfaithful. The triplet $\mathbf{3}_{1}^{l}$ differs in the overall sign of the generator $b$ from the representation $\mathbf{3}_{2}^{l}$. The generator $a$ is the same for all $l$ and the identities $c\left(\mathbf{3}_{k}^{l}\right)=c\left(\mathbf{3}_{k}^{1}\right)^{l}, d\left(\mathbf{3}_{k}^{l}\right)=d\left(\mathbf{3}_{k}^{1}\right)^{l}$ are fulfilled. It is clear that each power of the $c$ and $d$ generators will appear in every faithful three-dimensional irreducible representation. Without loss of generality, we shall embed the three left-handed lepton fields into a $\Delta\left(6 n^{2}\right)$ triplet $\mathbf{3}_{1}^{1}$ which is denoted as $\mathbf{3}$ in the following. For convenience we shall not distinguish the abstract elements of $\Delta\left(6 n^{2}\right)$ and their representation matrices hereafter.

The CP symmetry compatible with the $\Delta\left(6 n^{2}\right)$ flavor symmetry group has been analyzed in refs. $[55,56]$. It has been shown that the CP symmetry can be consistently defined in the presence of $\Delta\left(6 n^{2}\right)$ flavor symmetry if $n$ is not divisible by 3 . The viable CP transformations turn out to be of the same form as the flavor symmetry transformations in our working basis [56]. Moreover, the physically well defined CP transformations can also be implemented in a model for the case of $n=3 \mathbb{Z}$ if the model does not contain fields transforming as $\Delta\left(6 n^{2}\right)$ doublets $\mathbf{2}_{\mathbf{2}}, \mathbf{2}_{\mathbf{3}}$ and $\mathbf{2}_{\mathbf{4}}$ [56]. Now we determine the possible $Z_{2} \times C P$ subgroups of the $\Delta\left(6 n^{2}\right)$ and CP symmetries. The order two elements of the $\Delta\left(6 n^{2}\right)$ group are

$$
b c^{x} d^{x}, \quad a b c^{x}, \quad a^{2} b d^{x}, \quad x=0,1 \ldots n-1,
$$

and

$$
c^{n / 2}, \quad d^{n / 2}, \quad c^{n / 2} d^{n / 2},
$$

for even $n$. The three elements in eq. (3.3) are conjugate to each other, and the same holds true for the elements in eq. (3.4). As regards the residual CP transformation $X$, it has to be a unitary and symmetric matrix in order to avoid degenerate neutrino or charged lepton masses. Hence the admissible candidates for $X$ are

$$
c^{\gamma} d^{\delta}, \quad b c^{\gamma} d^{-\gamma}, \quad a b c^{\gamma} d^{2 \gamma}, \quad a^{2} b c^{2 \gamma} d^{\gamma}, \quad \gamma, \delta=0,1, \ldots, n-1 .
$$

Consistently combining the $Z_{2}$ subgroups generated by the elements in eqs. (3.3), (3.4) with the possible residual $\mathrm{CP}$ transformations in eq. (3.5), we can find all the viable $Z_{2} \times C P$ residual subgroups originating from $\Delta\left(6 n^{2}\right)$ and CP symmetries. Notice that the consistency condition of eq. (2.1) has to be fulfilled. Following the procedures presented in 
section 2, the corresponding Takagi factorization for each residual symmetry can be calculated, and all these results are summarized in table 1 . We see that the residual subgroup $Z_{2} \times C P$ can take nine different forms. As a consequence, there are $9 \times 9=81$ possible combinations of the residual symmetries $Z_{2}^{g_{l}} \times X_{l}$ and $Z_{2}^{g_{\nu}} \times X_{\nu}$ in the charged lepton and neutrino sectors. The different residual symmetries could be related by similarity transformations. After analyzing all those 81 possible combinations we find only 4 independent viable cases. Without loss of generality, we choose the 4 representative residual symmetries to be those shown in table 2. For each case the resulting prediction for the lepton mixing matrix can be straightforwardly obtained by using the master formula of eq. (2.12) and the Takagi factorization matrices listed in table 1. In particular, one can read off the unique element completely fixed by residual symmetry, as shown in table 2. The fixed element is actually only determined by the residual flavor symmetry, and it is given by the inner product of the eigenvectors with nondegenerate eigenvalues of $Z_{2}^{g_{l}}$ and $Z_{2}^{g_{\nu}}$.

The global analysis of the available neutrino oscillation data gives the following $3 \sigma$ ranges of the absolute values of the mixing matrix entries [8],

$$
\left\|U_{\mathrm{PMNS}}\right\|_{3 \sigma}=\left(\begin{array}{ccc}
0.800 \rightarrow 0.846 & 0.514 \rightarrow 0.582 & 0.139 \rightarrow 0.155 \\
0.209 \rightarrow 0.538 & 0.417 \rightarrow 0.720 & 0.613 \rightarrow 0.789 \\
0.219 \rightarrow 0.544 & 0.431 \rightarrow 0.729 & 0.597 \rightarrow 0.777
\end{array}\right)
$$

for normal mass hierarchy (NH) neutrino mass spectrum, and

$$
\left\|U_{\mathrm{PMNS}}\right\|_{3 \sigma}=\left(\begin{array}{lll}
0.800 \rightarrow 0.845 & 0.514 \rightarrow 0.582 & 0.140 \rightarrow 0.155 \\
0.206 \rightarrow 0.536 & 0.413 \rightarrow 0.716 & 0.619 \rightarrow 0.792 \\
0.223 \rightarrow 0.545 & 0.436 \rightarrow 0.732 & 0.593 \rightarrow 0.771
\end{array}\right)
$$

for inverted mass hierarchy (IH). Obviously we see that none element of the PMNS mixing matrix can be equal to 0 or 1 . Therefore out of all possibilities only four are possibly compatible with the present experimental data on lepton mixing. Then we proceed to study these four cases and their predictions for lepton mixing angles and $\mathrm{CP}$ violating phases one by one.

(I) $Z_{2}^{g_{l}}=Z_{2}^{b c^{x} d^{x}}, X_{l}=\left\{c^{\gamma} d^{-2 x-\gamma}, b c^{x+\gamma} d^{-x-\gamma}\right\}, Z_{2}^{g_{\nu}}=Z_{2}^{b c^{y} d^{y}}, X_{\nu}=$ $\left\{\boldsymbol{c}^{\boldsymbol{\delta}} \boldsymbol{d}^{-2 \boldsymbol{y}-\boldsymbol{\delta}}, \boldsymbol{b} \boldsymbol{c}^{\boldsymbol{y + \delta}} \boldsymbol{d}^{-\boldsymbol{y}-\boldsymbol{\delta}}\right\}$. In this case, we can easily read off the matrix $\Sigma \equiv \Sigma_{l}^{\dagger} \Sigma_{\nu}$ as follows,

$$
\Sigma=\left(\begin{array}{ccc}
\cos \varphi_{1} & -i \sin \varphi_{1} & 0 \\
-i \sin \varphi_{1} & \cos \varphi_{1} & 0 \\
0 & 0 & e^{i \varphi_{2}}
\end{array}\right)
$$

where an overall phase is omitted and the parameters $\varphi_{1}$ and $\varphi_{2}$ are given by

$$
\varphi_{1}=\frac{x-y}{n} \pi, \quad \varphi_{2}=\frac{3(x-y+\gamma-\delta)}{n} \pi .
$$

We find that the parameters $\varphi_{1}$ and $\varphi_{2}$ are independent of each other. Both the values of $\varphi_{1}$ and $\varphi_{2}$ are determined by the assumed remnant symmetries, and they can take the 


\begin{tabular}{|c|c|c|}
\hline$Z_{2}^{g_{l}}\left(Z_{2}^{g_{\nu}}\right)$ & $X_{l}\left(X_{\nu}\right)$ & $\Sigma_{l}\left(\Sigma_{\nu}\right)$ \\
\hline$Z_{2}^{b c^{x} d^{x}}$ & $\begin{array}{c}c^{\gamma} d^{-2 x-\gamma} \\
b c^{x+\gamma} d^{-x-\gamma}\end{array}$ & $\frac{1}{\sqrt{2}}\left(\begin{array}{ccc}-e^{\frac{i \pi \gamma}{n}} & e^{\frac{i \pi \gamma}{n}} & 0 \\
0 & 0 & -\sqrt{2} e^{-\frac{2 i \pi(x+\gamma)}{n}} \\
e^{\frac{i \pi(2 x+\gamma)}{n}} & e^{\frac{i \pi(2 x+\gamma)}{n}} & 0\end{array}\right)$ \\
\hline$Z_{2}^{a b c^{x}}$ & $\begin{array}{c}c^{\gamma} d^{2 x+2 \gamma} \\
a b c^{x+\gamma} d^{2 x+2 \gamma}\end{array}$ & $\frac{1}{\sqrt{2}}\left(\begin{array}{ccc}-e^{\frac{i \pi \gamma}{n}} & 0 & e^{\frac{i \pi \gamma}{n}} \\
e^{\frac{i \pi(2 x+\gamma)}{n}} & 0 & e^{\frac{i \pi(2 x+\gamma)}{n}} \\
0 & -\sqrt{2} e^{-\frac{2 i \pi(x+\gamma)}{n}} & 0\end{array}\right)$ \\
\hline$Z_{2}^{a^{2} b d^{x}}$ & $\begin{array}{c}c^{2 x+2 \gamma} d^{\gamma} \\
a^{2} b c^{2 x+2 \gamma} d^{x+\gamma}\end{array}$ & $\frac{1}{\sqrt{2}}\left(\begin{array}{ccc}0 & 0 & \sqrt{2} e^{\frac{2 i \pi(x+\gamma)}{n}} \\
-e^{-\frac{i \pi(2 x+\gamma)}{n}} & e^{-\frac{i \pi(2 x+\gamma)}{n}} & 0 \\
e^{-\frac{i \pi \gamma}{n}} & e^{-\frac{i \pi \gamma}{n}} & 0\end{array}\right)$ \\
\hline$Z^{c^{n / 2}}$ & $c^{\gamma} d^{\delta}$ & $\left(\begin{array}{ccc}0 & 0 & e^{\frac{i \pi \gamma}{n}} \\
0 & -e^{-\frac{i \pi(\gamma-\delta)}{n}} & 0 \\
e^{-\frac{i \pi \delta}{n}} & 0 & 0\end{array}\right)$ \\
\hline & $a b c^{\gamma} d^{2 \gamma}$ & $\frac{1}{\sqrt{2}}\left(\begin{array}{ccc}0 & i e^{\frac{i \pi \gamma}{n}} & e^{\frac{i \pi \gamma}{n}} \\
0 & -i e^{\frac{i \pi \gamma}{n}} & e^{\frac{i \pi \gamma}{n}} \\
\sqrt{2} e^{-\frac{2 i \pi \gamma}{n}} & 0 & 0\end{array}\right.$ \\
\hline \multirow{2}{*}{$Z_{2}^{d^{n / 2}}$} & $c^{\gamma} d^{\delta}$ & $\left(\begin{array}{ccc}e^{\frac{i \pi \gamma}{n}} & 0 & 0 \\
0 & 0 & -e^{-\frac{i \pi(\gamma-\delta)}{n}} \\
0 & e^{-\frac{i \pi \delta}{n}} & 0\end{array}\right)$ \\
\hline & $a^{2} b c^{2 \gamma} d^{\gamma}$ & $\frac{1}{\sqrt{2}}\left(\begin{array}{ccc}\sqrt{2} e^{\frac{2 i \pi \gamma}{n}} & 0 & 0 \\
0 & i e^{-\frac{i \pi \gamma}{n}} & e^{-\frac{i \pi \gamma}{n}} \\
0 & -i e^{-\frac{i \pi \gamma}{n}} & e^{-\frac{i \pi \gamma}{n}}\end{array}\right)$ \\
\hline \multirow{2}{*}{$Z_{2}^{c^{n / 2} d^{n / 2}}$} & $c^{\gamma} d^{\delta}$ & $\left(\begin{array}{ccc}0 & 0 & -e^{\frac{i \pi \gamma}{n}} \\
-e^{-\frac{i \pi(\gamma-\delta)}{n}} & 0 & 0 \\
0 & e^{-\frac{i \pi \delta}{n}} & 0\end{array}\right)$ \\
\hline & $b c^{\gamma} d^{-\gamma}$ & $\frac{1}{\sqrt{2}}\left(\begin{array}{ccc}0 & i e^{\frac{i \pi \gamma}{n}} & e^{\frac{i \pi \gamma}{n}} \\
\sqrt{2} e^{-\frac{2 i \pi \gamma}{n}} & 0 & 0 \\
0 & -i e^{\frac{i \pi \gamma}{n}} & e^{\frac{i \pi \gamma}{n}}\end{array}\right)$ \\
\hline
\end{tabular}

Table 1. The possible residual subgroups of the structure $Z_{2} \times C P$ and the corresponding Takagi factorization matrices, where the parameters $x, \gamma, \delta$ can take the values of $0,1, \ldots, n-1$. 


\begin{tabular}{|c|c|c|c|c|}
\hline$Z_{2}^{g_{l}}$ & $X_{l}$ & $Z_{2}^{g_{\nu}}$ & $X_{\nu}$ & Fixed element \\
\hline \multirow{3}{*}{$Z_{2}^{b c^{x} d^{x}}$} & \multirow{2}{*}{$c^{\gamma} d^{-2 x-\gamma}}$, & $Z_{2}^{b c^{y} d^{y}}$ & $\left\{c^{\delta} d^{-2 y-\delta}, b c^{y+\delta} d^{-y-\delta}\right\}$ & $\cos \varphi_{1}$ \\
\cline { 3 - 5 } & $\left.b c^{x+\gamma} d^{-x-\gamma}\right\}$ & $Z_{2}^{a b c^{y}}$ & $\left\{c^{\delta} d^{2 y+2 \delta}, a b c^{y+\delta} d^{2 y+2 \delta}\right\}$ & $\frac{1}{2}$ \\
\cline { 3 - 5 } & & $Z_{2}^{c^{n / 2}}$ & $c^{\alpha} d^{\delta}$ & $\frac{1}{\sqrt{2}}$ \\
\hline$Z_{2}^{c^{n / 2}}$ & $c^{\alpha} d^{\beta}$ & $Z_{2}^{b c^{x} d^{x}}$ & $\left\{c^{\gamma} d^{-2 x-\gamma}, b c^{x+\gamma} d^{-x-\gamma}\right\}$ & $\frac{1}{\sqrt{2}}$ \\
\hline
\end{tabular}

Table 2. The four viable combinations of residual symmetries with the structure $Z_{2} \times C P$ in the neutrino and charged lepton sectors, where the parameters $x, y, \alpha, \beta, \gamma$ and $\delta$ can take integer values between 0 and $n-1$. The angle $\varphi_{1}=(x-y) \pi / n$ is determined by the choice of residual symmetry. The entry completely fixed by residual symmetry is shown in the fifth column for each case.

following discrete values

$$
\begin{array}{ll}
\varphi_{1}(\bmod 2 \pi)=0, \frac{1}{n} \pi, \frac{2}{n} \pi, \ldots, \frac{2 n-1}{n} \pi, \\
\varphi_{2}(\bmod 2 \pi)=0, \frac{3}{n} \pi, \frac{6}{n} \pi, \ldots, \frac{2 n-3}{n} \pi, & 3 \mid n, \\
\varphi_{2}(\bmod 2 \pi)=0, \frac{1}{n} \pi, \frac{2}{n} \pi, \ldots, \frac{2 n-1}{n} \pi, & 3 \nmid n .
\end{array}
$$

Inserting the expression of $\Sigma$ into the master formula eq. (2.12), we find the lepton mixing matrix is determined to be

$$
U_{\mathrm{I}}=\left(\begin{array}{ccc}
\cos \varphi_{1} & s_{\nu} \sin \varphi_{1} & -c_{\nu} \sin \varphi_{1} \\
-s_{l} \sin \varphi_{1} & c_{l} c_{\nu} e^{i \varphi_{2}}+s_{l} s_{\nu} \cos \varphi_{1} & c_{l} s_{\nu} e^{i \varphi_{2}}-c_{\nu} s_{l} \cos \varphi_{1} \\
c_{l} \sin \varphi_{1} & c_{\nu} s_{l} e^{i \varphi_{2}}-c_{l} s_{\nu} \cos \varphi_{1} & s_{l} s_{\nu} e^{i \varphi_{2}}+c_{l} c_{\nu} \cos \varphi_{1}
\end{array}\right),
$$

up to permutations of rows and columns, where we have omitted the phases matrices $Q_{l}$ and $Q_{\nu}$ for notational simplicity, and the parameters $c_{l}, c_{\nu}, s_{l}$ and $s_{\nu}$ are abbreviations defined as

$$
c_{l} \equiv \cos \theta_{l}, \quad c_{\nu} \equiv \cos \theta_{\nu}, \quad s_{l} \equiv \sin \theta_{l}, \quad s_{\nu} \equiv \sin \theta_{\nu} .
$$

These notations would be frequently used in the following. Obviously one entry of the mixing matrix is fixed to be $\cos \varphi_{1}$ which is independent of $\theta_{l}$ and $\theta_{\nu}$. From the expression of $U_{\mathrm{I}}$, we know that it has the following symmetry properties,

$$
\begin{aligned}
& U_{\mathrm{I}}\left(\varphi_{1}+\pi, \varphi_{2}, \theta_{l}, \theta_{\nu}\right)=U_{\mathrm{I}}\left(\varphi_{1}, \varphi_{2}, \theta_{l}, \pi-\theta_{\nu}\right) \operatorname{diag}(-1,-1,1), \\
& U_{\mathrm{I}}\left(\pi-\varphi_{1}, \varphi_{2}, \theta_{l}, \theta_{\nu}\right)=\operatorname{diag}(-1,1,1) U_{\mathrm{I}}\left(\varphi_{1}, \varphi_{2}, \theta_{l}, \pi-\theta_{\nu}\right) \operatorname{diag}(1,-1,1), \\
& U_{\mathrm{I}}\left(\varphi_{1}, \varphi_{2}+\pi, \theta_{l}, \theta_{\nu}\right)=U_{\mathrm{I}}\left(\varphi_{1}, \varphi_{2}, \theta_{l}, \pi-\theta_{\nu}\right) \operatorname{diag}(1,1,-1), \\
& U_{\mathrm{I}}\left(\varphi_{1}, \pi-\varphi_{2}, \theta_{l}, \theta_{\nu}\right)=U_{\mathrm{I}}^{*}\left(\varphi_{1}, \varphi_{2}, \theta_{l}, \pi-\theta_{\nu}\right) \operatorname{diag}(1,1,-1) .
\end{aligned}
$$

Note that the above diagonal matrices can be absorbed into $Q_{l}$ and $Q_{\nu}$. In the following, we shall only present the absolute values of the sines of all CP phases. The reason is because the neutrino CP parity encoded in $Q_{\nu}$ could shift the Majorana phases $\alpha_{21}$ and $\alpha_{31}$ by $\pi$, 
and all the three $\mathrm{CP}$ phases $\delta_{\mathrm{CP}}, \alpha_{21}$ and $\alpha_{31}$ would become their opposite numbers when the three left-handed lepton doublets are embedded into the complex conjugated triplet $\overline{\mathbf{3}}$ instead of $\mathbf{3}$. As a result, without loss of generality, we could focus on the admissible values of $\varphi_{1}$ and $\varphi_{2}$ in the ranges of $0 \leq \varphi_{1} \leq \pi / 2$ and $0 \leq \varphi_{2} \leq \pi / 2$. As shown in section 2, the fixed element $\cos \varphi_{1}$ can be any of the nine elements of the PMNS mixing matrix, consequently the 36 possible permutations of rows and columns in general lead to nine independent mixing patterns which can be chosen to be

$$
\begin{array}{lll}
U_{\mathrm{I}, 1}=U_{\mathrm{I}}, & U_{\mathrm{I}, 2}=U_{\mathrm{I}} P_{12}, & U_{\mathrm{I}, 3}=U_{\mathrm{I}} P_{13}, \\
U_{\mathrm{I}, 4}=P_{12} U_{\mathrm{I}}, & U_{\mathrm{I}, 5}=P_{12} U_{\mathrm{I}} P_{12}, & U_{\mathrm{I}, 6}=P_{12} U_{\mathrm{I}} P_{13}, \\
U_{\mathrm{I}, 7}=P_{23} P_{12} U_{\mathrm{I}}, & U_{\mathrm{I}, 8}=P_{23} P_{12} U_{\mathrm{I}} P_{12}, & U_{\mathrm{I}, 9}=P_{23} P_{12} U_{\mathrm{I}} P_{13},
\end{array}
$$

where the explicit forms of permutation matrices $P_{12}, P_{13}$ and $P_{23}$ are given in eq. (2.15). Notice that $U_{\mathrm{I}, 7}, U_{\mathrm{I}, 8}$ and $U_{\mathrm{I}, 9}$ are related to $U_{\mathrm{I}, 4}, U_{\mathrm{I}, 5}$ and $U_{\mathrm{I}, 6}$ through an exchange of the second and third rows of the PMNS mixing matrix, respectively. It is well-known that the atmospheric mixing angle $\theta_{23}$ becomes $\pi / 2-\theta_{23}$, the Dirac CP phases $\delta_{\mathrm{CP}}$ becomes $\pi+\delta_{\mathrm{CP}}$ and the other mixing parameters are kept intact after the second and third rows of the PMNS matrix are permuted. Hence we shall not regard two mixing matrices related by the exchange of the second and third rows as being independent hereinafter. It is sufficient to only give the mixing parameters for $U_{\mathrm{I}, 4}, U_{\mathrm{I}, 5}$ and $U_{\mathrm{I}, 6}$, then the mixing parameters of $U_{\mathrm{I}, 7}, U_{\mathrm{I}, 8}$ and $U_{\mathrm{I}, 9}$ can be easily read out. For each independent mixing pattern we can straightforwardly extract the expressions of the mixing angles $\sin ^{2} \theta_{13}, \sin ^{2} \theta_{12}, \sin ^{2} \theta_{23}$ and the CP invariants $J_{\mathrm{CP}}, I_{1}, I_{2}$ in the usual way. All theses results are collected in table 3. Here $J_{\mathrm{CP}}$ is the Jarlskog invariant [71]

$$
J_{\mathrm{CP}}=\Im\left(U_{11} U_{33} U_{13}^{*} U_{31}^{*}\right)=\frac{1}{8} \sin 2 \theta_{12} \sin 2 \theta_{13} \sin 2 \theta_{23} \cos \theta_{13} \sin \delta_{\mathrm{CP}},
$$

and the rephasing invariants $I_{1}$ and $I_{2}$ are related with the Majorana CP phases [72-75]

$$
\begin{aligned}
& I_{1}=\Im\left(U_{11}^{* 2} U_{12}^{2}\right)=\frac{1}{4} \sin ^{2} 2 \theta_{12} \cos ^{4} \theta_{13} \sin \alpha_{21}, \\
& I_{2}=\Im\left(U_{11}^{* 2} U_{13}^{2}\right)=\frac{1}{4} \sin ^{2} 2 \theta_{13} \cos ^{2} \theta_{12} \sin \left(\alpha_{31}-2 \delta_{\mathrm{CP}}\right),
\end{aligned}
$$

where $\delta_{\mathrm{CP}}$ is the Dirac $\mathrm{CP}$ violation phase, $\alpha_{21}$ and $\alpha_{31}$ are the Majorana $\mathrm{CP}$ phases in the standard parameterization of the lepton mixing matrix [1]. We see that the mixing parameters depend on the continuous parameters $\theta_{l}$ and $\theta_{\nu}$ as well as the discrete parameters $\varphi_{1}$ and $\varphi_{2}$. As a consequence, sum rules among the mixing angles and the Dirac CP phase $\delta_{\mathrm{CP}}$ can be found as follows:

$$
\begin{array}{ll}
U_{\mathrm{I}, 1}: & \cos ^{2} \theta_{12} \cos ^{2} \theta_{13}=\cos ^{2} \varphi_{1}, \quad U_{\mathrm{I}, 2}: \quad \sin ^{2} \theta_{12} \cos ^{2} \theta_{13}=\cos ^{2} \varphi_{1}, \\
U_{\mathrm{I}, 6}: & \sin ^{2} \theta_{23} \cos ^{2} \theta_{13}=\cos ^{2} \varphi_{1}, \quad U_{\mathrm{I}, 9}: \quad \cos ^{2} \theta_{23} \cos ^{2} \theta_{13}=\cos ^{2} \varphi_{1}, \\
U_{\mathrm{I}, 4}: & \cos \delta_{\mathrm{CP}}=\frac{2\left(\cos ^{2} \varphi_{1}-\sin ^{2} \theta_{12} \cos ^{2} \theta_{23}-\sin ^{2} \theta_{13} \cos ^{2} \theta_{12} \sin ^{2} \theta_{23}\right)}{\sin 2 \theta_{12} \sin \theta_{13} \sin 2 \theta_{23}},
\end{array}
$$




\begin{tabular}{|c|c|c|c|}
\hline & & & Case I \\
\hline PMNS & $\varphi_{1} / \pi$ & $n_{\min }$ & Mixing Parameters \\
\hline \multirow{4}{*}{$U_{\mathrm{I}, 1}$} & \multirow{4}{*}[0.179,0.205]{} & \multirow{4}{*}{5} & $\sin ^{2} \theta_{13}=c_{\nu}^{2} \sin ^{2} \varphi_{1}$ \\
\hline & & & $\sin ^{2} \theta_{12}=\frac{s_{\nu}^{2} \sin ^{2} \varphi_{1}}{1-c_{\nu}^{2} \sin ^{2} \varphi_{1}}$ \\
\hline & & & $\sin ^{2} \theta_{23}=\frac{c_{\nu}^{2} s_{l}^{2} \cos ^{2} \varphi_{1}+c_{l}^{2} s_{\nu}^{2}-\mathcal{X}_{1} \cos \varphi_{2}}{1-c_{\nu}^{2} \sin ^{2} \varphi_{1}}$ \\
\hline & & & $I_{1}=I_{2}=0$ \\
\hline \multirow{4}{*}{$U_{\mathrm{I}, 2}$} & \multirow{4}{*}[0.302,0.328]{} & \multirow{4}{*}{13} & $\sin ^{2} \theta_{13}=c_{\nu}^{2} \sin ^{2} \varphi_{1}$ \\
\hline & & & $\sin ^{2} \theta_{12}=\frac{\cos ^{2} \varphi_{1}}{1-c_{\nu}^{2} \sin ^{2} \varphi_{1}}$ \\
\hline & & & $\sin ^{2} \theta_{23}=\frac{c_{\nu}^{2} s_{l}^{2} \cos ^{2} \varphi_{1}+c_{l}^{2} s_{\nu}^{2}-\mathcal{X}_{1} \cos \varphi_{2}}{1-c_{\nu}^{2} \sin ^{2} \varphi_{1}}$ \\
\hline & & & $I_{1}=I_{2}=0$ \\
\hline \multirow{4}{*}{$U_{\mathrm{I}, 3}$} & \multirow{4}{*}[0.450,0.456]{} & \multirow{4}{*}{11} & $\sin ^{2} \theta_{13}=\cos ^{2} \varphi_{1}$ \\
\hline & & & $\sin ^{2} \theta_{12}=s_{\nu}^{2}$ \\
\hline & & & $\sin ^{2} \theta_{23}=s_{l}^{2}$ \\
\hline & & & $I_{1}=I_{2}=0$ \\
\hline \multirow{5}{*}{$U_{\mathrm{I}, 4}$} & \multirow{5}{*}[0.319,0.433]{} & \multirow{5}{*}{3} & $\sin ^{2} \theta_{13}=c_{\nu}^{2} s_{l}^{2} \cos ^{2} \varphi_{1}+c_{l}^{2} s_{\nu}^{2}-\mathcal{X}_{1} \cos \varphi_{2}$ \\
\hline & & & $\sin ^{2} \theta_{12}=1-\frac{s_{l}^{2} \sin ^{2} \varphi_{1}}{1-c_{\nu}^{2} s_{l}^{2} \cos ^{2} \varphi_{1}-c_{l}^{2} s_{\nu}^{2}+\mathcal{X}_{1} \cos \varphi_{2}}$ \\
\hline & & & $\sin ^{2} \theta_{23}=\frac{c_{\nu}^{2} \sin ^{2} \varphi_{1}}{1-c_{\nu}^{2} s_{l}^{2} \cos ^{2} \varphi_{1}-c_{l}^{2} s_{\nu}^{2}+\mathcal{X}_{1} \cos \varphi_{2}}$ \\
\hline & & & $\left|I_{1}\right|=\left|2 c_{l} c_{\nu} s_{l}^{2} \sin ^{2} \varphi_{1} \sin \varphi_{2}\left(c_{l} c_{\nu} \cos \varphi_{2}+s_{l} s_{\nu} \cos \varphi_{1}\right)\right|$ \\
\hline & & & $\left|I_{2}\right|=\left|2 c_{l} s_{l}^{2} s_{\nu} \sin ^{2} \varphi_{1} \sin \varphi_{2}\left(c_{l} s_{\nu} \cos \varphi_{2}-c_{\nu} s_{l} \cos \varphi_{1}\right)\right|$ \\
\hline \multirow{5}{*}{$U_{\mathrm{I}, 5}$} & \multirow{5}{*}[0.244,0.363]{} & \multirow{5}{*}{3} & $\sin ^{2} \theta_{13}=c_{\nu}^{2} s_{l}^{2} \cos ^{2} \varphi_{1}+c_{l}^{2} s_{\nu}^{2}-\mathcal{X}_{1} \cos \varphi_{2}$ \\
\hline & & & $\sin ^{2} \theta_{12}=\frac{s_{l}^{2} \sin ^{2} \varphi_{1}}{1-c_{\nu}^{2} s_{l}^{2} \cos ^{2} \varphi_{1}-c_{l}^{2} s_{\nu}^{2}+\mathcal{X}_{1} \cos \varphi_{2}}$ \\
\hline & & & $\sin ^{2} \theta_{23}=\frac{c_{\nu}^{2} \sin ^{2} \varphi_{1}}{1-c_{\nu}^{2} s_{l}^{2} \cos ^{2} \varphi_{1}-c_{l}^{2} s_{\nu}^{2}+\mathcal{X}_{1} \cos \varphi_{2}}$ \\
\hline & & & $\left|I_{1}\right|=\left|2 c_{l} c_{\nu} s_{l}^{2} \sin ^{2} \varphi_{1} \sin \varphi_{2}\left(c_{l} c_{\nu} \cos \varphi_{2}+s_{l} s_{\nu} \cos \varphi_{1}\right)\right|$ \\
\hline & & & $\left|I_{2}\right|=\left|\mathcal{X}_{1} \sin \varphi_{2}\left(c_{l}^{2}-s_{l}^{2} \cos ^{2} \varphi_{1}-2 s_{l} c_{l} \cot 2 \theta_{\nu} \cos \varphi_{1} \cos \varphi_{2}\right)\right|$ \\
\hline \multirow{5}{*}{$U_{\mathrm{I}, 6}$} & \multirow{5}{*}[0.211,0.290]{} & \multirow{5}{*}{4} & $\sin ^{2} \theta_{13}=s_{l}^{2} \sin ^{2} \varphi_{1}$ \\
\hline & & & $\sin ^{2} \theta_{12}=\frac{c_{l}^{2} c_{\nu}^{2}+s_{l}^{2} s_{\nu}^{2} \cos ^{2} \varphi_{1}+\mathcal{X}_{1} \cos \varphi_{2}}{1-s_{l}^{2} \sin ^{2} \varphi_{1}}$ \\
\hline & & & $\sin ^{2} \theta_{23}=\frac{\cos ^{2} \varphi_{1}}{1-s_{l}^{2} \sin ^{2} \varphi_{1}}$ \\
\hline & & & $\left|I_{1}\right|=\left|\mathcal{X}_{1} \sin \varphi_{2}\left(2 s_{l} c_{l} \cot 2 \theta_{\nu} \cos \varphi_{1} \cos \varphi_{2}+s_{l}^{2} \cos ^{2} \varphi_{1}-c_{l}^{2}\right)\right|$ \\
\hline & & & $\left|I_{2}\right|=\left|2 c_{l} s_{l}^{2} s_{\nu} \sin ^{2} \varphi_{1} \sin \varphi_{2}\left(c_{\nu} s_{l} \cos \varphi_{1}-c_{l} s_{\nu} \cos \varphi_{2}\right)\right|$ \\
\hline
\end{tabular}

Table 3. The predictions of the mixing parameters for all the nine permutations of the mixing matrix in the case I. The magnitude of $J_{\mathrm{CP}}$ is identical for all the nine mixing patterns, i.e. $\left|J_{\mathrm{CP}}\right|=$ $\left|c_{l} c_{\nu} s_{l} s_{\nu} \sin ^{2} \varphi_{1} \cos \varphi_{1} \sin \varphi_{2}\right|$. The parameter $\mathcal{X}_{1}$ is defined as $\mathcal{X}_{1}=\frac{1}{2} \sin 2 \theta_{l} \sin 2 \theta_{\nu} \cos \varphi_{1}$. The admissible range of $\varphi_{1}$ in the second column is obtained from the requirement that the fixed element $\cos \varphi_{1}$ is in the experimentally preferred $3 \sigma$ range. The notation $n_{\min }$ denotes the smallest value of the group index $n$ which can give a good fit to the experimental data [8]. Here the neutrino mass spectrum is assumed to be $\mathrm{NH}$, and the range of $\varphi_{1}$ would change a little for $\mathrm{IH}$. 


$$
\begin{aligned}
& U_{\mathrm{I}, 5}: \quad \cos \delta_{\mathrm{CP}}=-\frac{2\left(\cos ^{2} \varphi_{1}-\cos ^{2} \theta_{12} \cos ^{2} \theta_{23}-\sin ^{2} \theta_{13} \sin ^{2} \theta_{12} \sin ^{2} \theta_{23}\right)}{\sin 2 \theta_{12} \sin \theta_{13} \sin 2 \theta_{23}}, \\
& U_{\mathrm{I}, 7}: \quad \cos \delta_{\mathrm{CP}}=-\frac{2\left(\cos ^{2} \varphi_{1}-\sin ^{2} \theta_{12} \sin ^{2} \theta_{23}-\sin ^{2} \theta_{13} \cos ^{2} \theta_{12} \cos ^{2} \theta_{23}\right)}{\sin 2 \theta_{12} \sin \theta_{13} \sin 2 \theta_{23}}, \\
& U_{\mathrm{I}, 8}: \quad \cos \delta_{\mathrm{CP}}=\frac{2\left(\cos ^{2} \varphi_{1}-\cos ^{2} \theta_{12} \sin ^{2} \theta_{23}-\sin ^{2} \theta_{13} \sin ^{2} \theta_{12} \cos ^{2} \theta_{23}\right)}{\sin 2 \theta_{12} \sin \theta_{13} \sin 2 \theta_{23}}
\end{aligned}
$$

For a given value of $\varphi_{1}$, the possible ranges of $\cos \delta_{\mathrm{CP}}$ can be obtained from the above correlations by varying the mixing angles over their $3 \sigma$ ranges [8]. Thus these sum rules could be tested and possibly distinguished from each other in future neutrino oscillation experiments if the Dirac CP phase $\delta_{\mathrm{CP}}$ is measured. In this case, the assumed residual symmetry determines one entry of the mixing matrix to be $\cos \varphi_{1}$, consequently the constraint on the parameter $\varphi_{1}$ can be obtained by requiring $\cos \varphi_{1}$ in the experimentally preferred $3 \sigma$ region, as shown in the second column of table 3. Furthermore we perform a comprehensive numerical analysis of the $\Delta\left(6 n^{2}\right)$ group, and find out the smallest value of the index $n$ of the group which can accommodate the experimental data on mixing angles for certain values of the parameters $\theta_{l}$ and $\theta_{\nu}$. In particularly, we find that it is sufficient to consider groups with the index $n \leq 13$. Since the first row of the PMNS mixing matrices $U_{\mathrm{I}, 1}, U_{\mathrm{I}, 2}$ and $U_{\mathrm{I}, 3}$ is real, both Majorana invariants $I_{1}$ and $I_{2}$ are vanishing for any values of $n$. Furthermore, we find that the smallest $n_{\min }$ with non-vanishing $I_{1}$ and $I_{2}$ is 4 for the mixing matrices $U_{\mathrm{I}, 6}$ and $U_{\mathrm{I}, 9}$, it is 5 for $U_{\mathrm{I}, 4}$ and $U_{\mathrm{I}, 7}$, and it is 10 for $U_{\mathrm{I}, 5}$ and $U_{\mathrm{I}, 8}$.

For the case of $n=3$, the parameter $\varphi_{2}$ only can take the value of 0 , the experimental data on mixing angles can be accommodated. However, $X=b c^{x+\gamma} d^{-x-\gamma}$ is an accidental CP symmetry common to the charged lepton and neutrino sector, the PMNS matrix is real, consequently all the three $\mathrm{CP}$ phases are trivial. Since the evidence of maximal Dirac CP phase $\delta_{\mathrm{CP}} \simeq 3 \pi / 2$ is reported by T2K and NOvA [3-6], as a concrete example we shall consider the $\Delta\left(6 \cdot 4^{2}\right)=\Delta(96)$ flavor group with $n=4$. In this case, the possible values of the parameters $\varphi_{1,2}$ are

$$
\varphi_{1}=0, \frac{\pi}{4}, \frac{\pi}{2}, \quad \varphi_{2}=0, \frac{\pi}{4}, \frac{\pi}{2},
$$

in the fundamental regions of $\varphi_{1} \in[0, \pi / 2]$ and $\varphi_{2} \in[0, \pi / 2]$. The case of $\varphi_{1}=\pi / 4$ can give rise to a phenomenological viable mixing pattern, and accordingly the fixed element is equal to $\cos \varphi_{1}=1 / \sqrt{2}$ which can be the (22), (23), (32) or (33) entries of the lepton mixing matrix. As a consequence, out of the nine permutations in eq. (3.14) only $U_{\mathrm{I}, 5}, U_{\mathrm{I}, 6}$, $U_{\mathrm{I}, 8}$ and $U_{\mathrm{I}, 9}$ could describe the experimental data. The predictions of $U_{\mathrm{I}, 8}$ and $U_{\mathrm{I}, 9}$ can be easily obtained from the results of $U_{\mathrm{I}, 5}$ and $U_{\mathrm{I}, 6}$ respectively, since they are related through the exchange of the second and third rows of the mixing matrices. In this case, all the three mixing angles $\theta_{12}, \theta_{13}, \theta_{23}$ and the CP violation phases $\delta_{\mathrm{CP}}, \alpha_{21}, \alpha_{31}$ only depend on two continuous parameters $\theta_{l}$ and $\theta_{\nu}$. The values of $\theta_{l, \nu}$ can be determined from the measured values of any two lepton mixing angles, then one can predict the third mixing angle and the $\mathrm{CP}$ violation phases. In order to see clearly whether the measured values of the mixing angles can be accommodated, we display the contour regions for the $3 \sigma$ intervals of $\sin ^{2} \theta_{i j}$ and their experimental best fit values in the plane $\theta_{\nu}$ versus $\theta_{l}$ in figure 1 , where 


\begin{tabular}{|c|c|c|c|c|c|c|c|c|c|c|c|}
\hline \multicolumn{12}{|c|}{ Case I with $n=4$} \\
\hline & $\varphi_{1}$ & $\varphi_{2}$ & $\theta_{l}^{\mathrm{bf}} / \pi$ & $\theta_{\nu}^{\mathrm{bf}} / \pi$ & $\chi_{\min }^{2}$ & $\sin ^{2} \theta_{13}$ & $\sin ^{2} \theta_{12}$ & $\sin ^{2} \theta_{23}$ & $\left|\sin \delta_{\mathrm{CP}}\right|$ & $\left|\sin \alpha_{21}\right|$ & $\left|\sin \alpha_{31}\right|$ \\
\hline \multirow{3}{*}{$U_{\mathrm{I}, 6}$} & \multirow{3}{*}{$\frac{\pi}{4}$} & \multirow{2}{*}{$\frac{\pi}{4}$} & \multirow{3}{*}{$\begin{array}{c}0.0667 \\
(0.0670)\end{array}$} & $\begin{array}{c}0.349 \\
(0.349)\end{array}$ & \multirow{3}{*}{$\begin{array}{c}6.734 \\
(9.989)\end{array}$} & \multirow{3}{*}{$\begin{array}{c}0.0216 \\
(0.0218)\end{array}$} & \multirow{3}{*}{$\begin{array}{c}0.306 \\
(0.306)\end{array}$} & \multirow{3}{*}{$\begin{array}{c}0.511 \\
(0.511)\end{array}$} & $\begin{array}{c}0.623 \\
(0.623)\end{array}$ & \multirow{2}{*}{$\begin{array}{c}0.440 \\
(0.442)\end{array}$} & $\begin{array}{c}0.333 \\
(0.335)\end{array}$ \\
\hline & & & & $\begin{array}{c}0.719 \\
(0.719)\end{array}$ & & & & & $\begin{array}{c}0.753 \\
(0.753)\end{array}$ & & $\begin{array}{c}0.290 \\
(0.292)\end{array}$ \\
\hline & & $\frac{\pi}{2}$ & & $\begin{array}{c}0.317 \\
(0.317)\end{array}$ & & & & & $\begin{array}{c}0.992 \\
(0.991)\end{array}$ & $\begin{array}{c}0.605 \\
(0.607)\end{array}$ & $\begin{array}{c}0.439 \\
(0.441)\end{array}$ \\
\hline
\end{tabular}

Table 4. Results of the $\chi^{2}$ analysis for $n=4$ in case I. The $\chi^{2}$ function obtains a global minimum $\chi_{\text {min }}^{2}$ at the best fit values $\left(\theta_{l}, \theta_{\nu}\right)=\left(\theta_{l}^{\text {bf }}, \theta_{\nu}^{\text {bf }}\right)$. We display the values of the mixing angles and $\mathrm{CP}$ violation phases at the best fitting point. The same values of mixing parameters as well as $\chi_{\min }^{2}$ are achieved at $\left(\theta_{l}, \theta_{\nu}\right)=\left(\pi-\theta_{l}^{\mathrm{bf}}, \pi-\theta_{\nu}^{\mathrm{bf}}\right)$, because the formulae of the mixing angles and CP invariants in table 3 are not changed, if $\theta_{l}$ and $\theta_{\nu}$ are replaced by $\pi-\theta_{l}$ and $\pi-\theta_{\nu}$ respectively. The numbers given in parentheses are the corresponding results for the inverted hierarhy neutrino mass spectrum. Although both mixing patterns $U_{\mathrm{I}, 5}$ and $U_{\mathrm{I}, 6}$ for $\varphi_{2}=0$ can accommodate the experimental data on mixing angles, we don't display the corresponding results, because all three $\mathrm{CP}$ phases are trivial which is disfavored by the evidence of $\delta_{\mathrm{CP}}=3 \pi / 2[3-6]$.

we use the data from the global fit of [8]. We find that only the mixing matrix $U_{\mathrm{I}, 5}$ with $\varphi_{2}=0$ and the mixing matrix $U_{\mathrm{I}, 6}$ with $\varphi_{2}=0, \pi / 4$ and $\pi / 2$ are viable. The value $\varphi_{2}=0$ leads to three trivial CP phases, consequently we will not show the corresponding results. Furthermore, we perform a conventional $\chi^{2}$ analysis, and the numerical results are reported in table 4 . We see that the mixing angles are quite close to the best fit values of global data analysis for particular choices of $\theta_{l, \nu}$. Notice that the expressions of the mixing angles are invariant under the transformations $\theta_{l} \rightarrow \pi-\theta_{l}$ and $\theta_{\nu} \rightarrow \pi-\theta_{\nu}$. Therefore the same results would be obtained at the point $\left(\theta_{l}, \theta_{\nu}\right)=\left(\pi-\theta_{l}^{\mathrm{bf}}, \pi-\theta_{\nu}^{\mathrm{bf}}\right)$, as indicated in figure 1. Since the neutrino mass ordering is unknown at present, we present the results of $\chi^{2}$ analysis for both $\mathrm{NH}$ and IH in table 4 . Although the global minimum $\chi_{\min }^{2}$ is a bit large for few cases, e.g. $\chi_{\min }^{2}=65.188>27$ for the mixing pattern $U_{\mathrm{III}, 1}$ and IH spectrum, the resulting lepton mixing angles can still be compatible with the experimental data at $3 \sigma$ level. The reason is that the one-dimensional $\chi^{2}$ projection of $\sin ^{2} \theta_{23}$ doesn't exactly follow the normal distribution [7-9]. Furthermore, the predictions for the CP violating phases $\left|\sin \delta_{\mathrm{CP}}\right|,\left|\sin \alpha_{21}\right|$ and $\left|\sin \alpha_{31}\right|$ are plotted in figure 2. From figure 1, we can see that the measured values of the mixing angles can be achieved in a quite small region of the $\theta_{\nu}-\theta_{l}$ plane. Hence the mixing angles and CP phases should be able to only vary a little around the numerical values listed in table 4 , and consequently the present approach is very predictive. Moreover, when all three mixing angles lie in their $3 \sigma$ ranges, for the mixing pattern $U_{\mathrm{I}, 6}$ with $\varphi_{1}=\pi / 4$, we find the absolute values of sines of the three CP phases are in the intervals

$$
\left|\sin \delta_{\mathrm{CP}}\right| \in[0.606,0.644] \cup[0.738,0.768], \quad\left|\sin \alpha_{21}\right| \in[0.405,0.477], \quad\left|\sin \alpha_{31}\right| \in[0.251,0.333],
$$



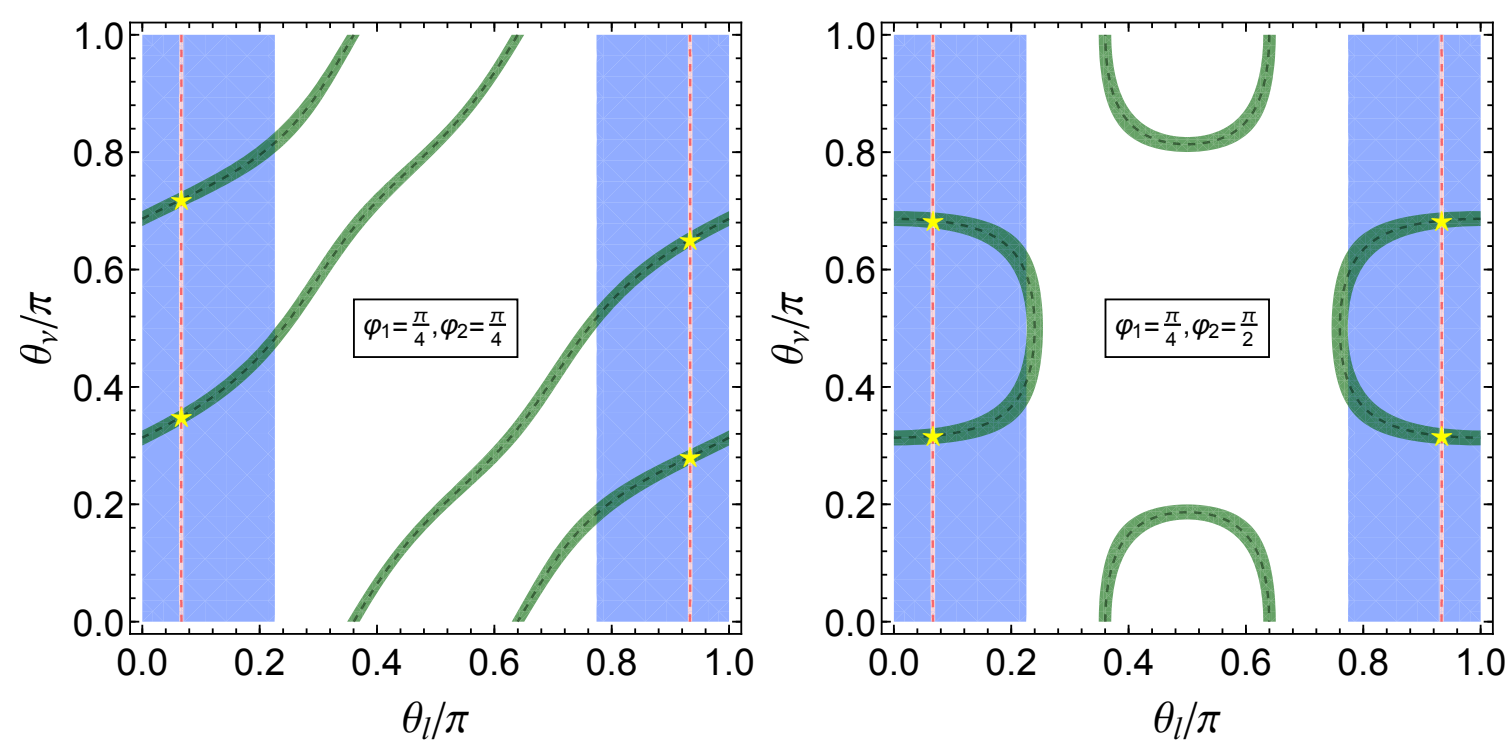

Figure 1. Contour plots of $\sin ^{2} \theta_{i j}$ in the $\theta_{\nu}-\theta_{l}$ plane for mixing matrix $U_{\mathrm{I}, 6}$ with $n=4$. The panels in the left and right sides are for $\left(\varphi_{1}, \varphi_{2}\right)=(\pi / 4, \pi / 4)$ and $(\pi / 4, \pi / 2)$ respectively. The red, green and blue areas denote the $3 \sigma$ contour regions of $\sin ^{2} \theta_{13}, \sin ^{2} \theta_{12}$ and $\sin ^{2} \theta_{23}$ respectively. The dashed contour lines represent the corresponding experimental best fit values. The $3 \sigma$ ranges as well as the best fit values of the mixing angles are adapted from [8]. The best fitting values of $\theta_{l, \nu}$ are indicated with yellow pentagrams.

for $\varphi_{2}=\pi / 4$ and

$$
\left|\sin \delta_{\mathrm{CP}}\right| \in[0.986,0.996], \quad\left|\sin \alpha_{21}\right| \in[0.559,0.652], \quad\left|\sin \alpha_{31}\right| \in[0.381,0.500],
$$

for $\varphi_{2}=\pi / 2$. It is interesting that we can obtain approximately maximal $\delta_{\mathrm{CP}}$ for $\varphi_{2}=$ $\pi / 2$. From table 4 , we see that the mixing patterns $U_{\mathrm{I}, 6}$ with $\left(\varphi_{1}, \varphi_{2}\right)=(\pi / 4, \pi / 4)$ and $\left(\varphi_{1}, \varphi_{2}\right)=(\pi / 4, \pi / 2)$ give rise to the same lepton mixing angles, but the predictions for Dirac CP violation phase and Majorna phases are different. Recently T2K and NOvA have reported a slight preference for $\delta_{\mathrm{CP}}$ close to $3 \pi / 2[3-6]$. T2K and NOvA can contribute to the measurement of the Dirac phase $\delta_{\mathrm{CP}}$, if running in both the neutrino and the antineutrino modes. They can possibly exclude certain ranges of $\delta_{\mathrm{CP}}$ especially the values around $\delta_{\mathrm{CP}}= \pm \pi / 2$, depending on $\theta_{23}$ and the neutrino mass hierarchy. Future longbaseline experiments such as DUNE [76-79], T2HK [80, 81], T2HKK [82] and possibly $\mathrm{ESS} \nu \mathrm{SB}[83,84]$ at the European Spallation Source will allow for a measurement of the Dirac phase and atmospheric mixing angle with significantly improved sensitivities and thus a discrimination between these two mixing patterns might be possible.

The neutrinoless double beta $\left((\beta \beta)_{0 \nu^{-}}\right)$decay $(A, Z) \rightarrow(A, Z+2)+e^{-}+e^{-}$is an important probe for the Majorana nature of the neutrinos. If this rare lepton number violating process was observed in future, neutrinos must be Majorana particles. In addition, it can also help us to determine the neutrino mass spectrum and at least can constraint the $\mathrm{CP}$ violating phases, if the associated nuclear matrix element is known precisely enough. 

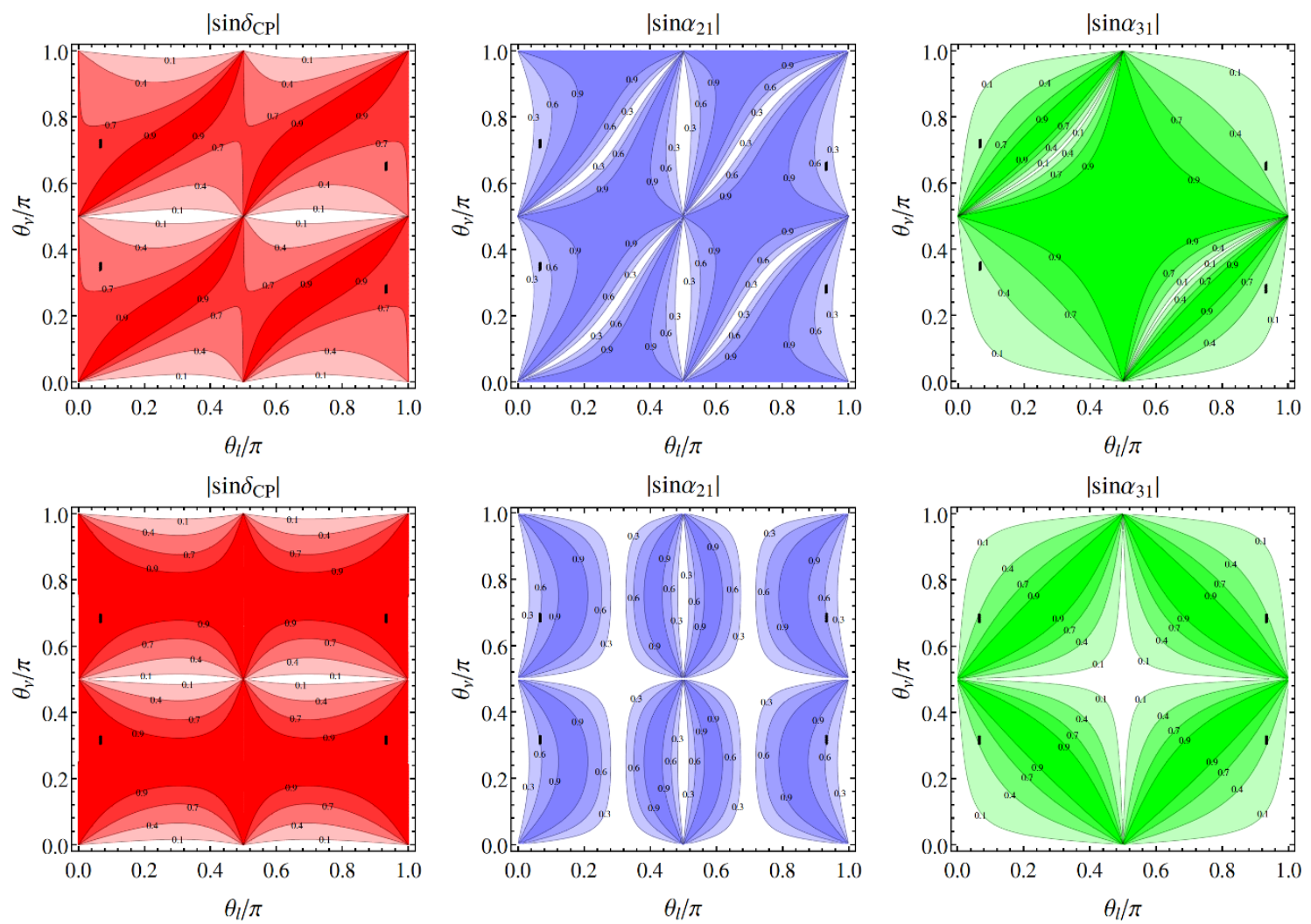

Figure 2. The contour plots of the $\mathrm{CP}$ violation phases $\left|\sin \delta_{\mathrm{CP}}\right|,\left|\sin \alpha_{21}\right|$ and $\left|\sin \alpha_{31}\right|$ for the mixing pattern $U_{\mathrm{I}, 6}$, where the parameters $\left(\varphi_{1}, \varphi_{2}\right)$ are equal to $(\pi / 4, \pi / 4)$ in the upper panels and $(\pi / 4, \pi / 2)$ in the lower panels. The black areas represent the regions for which all the three lepton mixing angles lie in their corresponding experimentally allowed $3 \sigma$ intervals [8]. As $U_{\mathrm{I}, 6}$ and $U_{\mathrm{I}, 9}$ are related through the exchange of the second and the third rows of the mixing matrix, they lead to the same Majorana phases $\alpha_{21}$ and $\alpha_{31}$ while the Dirac phase changes from $\delta_{\mathrm{CP}}$ to $\pi+\delta_{\mathrm{CP}}$.

The $(\beta \beta)_{0 \nu}$-decay amplitude is proportional to the effective Majorana mass $m_{e e}$ given by [1]

$$
m_{e e}=\left|m_{1} \cos ^{2} \theta_{12} \cos ^{2} \theta_{13}+m_{2} \sin ^{2} \theta_{12} \cos ^{2} \theta_{13} e^{i \alpha_{21}}+m_{3} \sin ^{2} \theta_{13} e^{i \alpha_{31}^{\prime}}\right| .
$$

where $\alpha_{31}^{\prime}=\alpha_{31}-2 \delta_{\mathrm{CP}}$. The light neutrino masses $m_{1,2,3}$ can be expressed in terms of the lightest neutrino mass $m_{\min }$ and the measured neutrino mass squared splittings $\Delta m_{21}^{2} \equiv m_{2}^{2}-m_{1}^{2}$ and $\Delta m_{3 \ell}^{2} \equiv m_{3}^{2}-m_{\ell}^{2}$ with $\ell=1$ for NH and $\ell=2$ for IH [8]. At present, the most stringent bound is set by the EXO-200 [85, 86] and KamLAND-ZEN [87],

$$
m_{e e}<(0.12-0.25) \mathrm{eV}
$$

at $90 \%$ confidence level. In the limit of vanishing lightest neutrino mass $m_{\min } \rightarrow 0$, we have

$$
\begin{aligned}
m_{e e} & \simeq\left|\sqrt{\Delta m_{21}^{2}} \sin ^{2} \theta_{12} \cos ^{2} \theta_{13} e^{i \alpha_{21}}+\sqrt{\Delta m_{31}^{2}} \sin ^{2} \theta_{13} e^{i \alpha_{31}^{\prime}}\right| \\
& =\left[\Delta m_{21}^{2} \sin ^{4} \theta_{12} \cos ^{4} \theta_{13}+\Delta m_{31}^{2} \sin ^{4} \theta_{13}+2 \sqrt{\Delta m_{21}^{2} \Delta m_{31}^{2}} \sin ^{2} \theta_{12} \cos ^{2} \theta_{13} \sin ^{2} \theta_{13} \cos \left(\alpha_{21}-\alpha_{31}^{\prime}\right)\right]^{1 / 2},
\end{aligned}
$$


for $\mathrm{NH}$ and

$$
\begin{aligned}
m_{e e} & \simeq\left|\sqrt{-\Delta m_{32}^{2}-\Delta m_{21}^{2}} \cos ^{2} \theta_{12} \cos ^{2} \theta_{13}+\sqrt{-\Delta m_{32}^{2}} \sin ^{2} \theta_{12} \cos ^{2} \theta_{13} e^{i \alpha_{21}}\right| \\
& \simeq\left|\cos ^{2} \theta_{12}+\sin ^{2} \theta_{12} e^{i \alpha_{21}}\right| \cos ^{2} \theta_{13} \sqrt{-\Delta m_{32}^{2}} \\
& =\sqrt{1-\sin ^{2} 2 \theta_{12} \sin ^{2} \frac{\alpha_{21}}{2}} \cos ^{2} \theta_{13} \sqrt{-\Delta m_{32}^{2}}
\end{aligned}
$$

for IH. In the case of quasi-degenerate spectrum $m_{\min }^{2} \gg \Delta m_{21}^{2},\left|\Delta m_{3 \ell}^{2}\right|$, we have

$$
m_{e e} \simeq \sqrt{1-\sin ^{2} 2 \theta_{12} \sin ^{2} \frac{\alpha_{21}}{2}} m_{\min }
$$

where the term proportional to $\sin ^{2} \theta_{13}$ is omitted. For the interesting mixing pattern $U_{\mathrm{I}, 6}$, the effective Majorana neutrino mass reads as

$$
m_{e e}=\left|\left(c_{l} s_{\nu} e^{i \varphi_{2}}-c_{\nu} s_{l} \cos \varphi_{1}\right)^{2} m_{1}+k_{1}\left(c_{l} c_{\nu} e^{i \varphi_{2}}+s_{l} s_{\nu} \cos \varphi_{1}\right)^{2} m_{2}+k_{2} s_{l}^{2} \sin ^{2} \varphi_{1} m_{3}\right|
$$

where $k_{1}, k_{2}= \pm 1$ arises from the ambiguity of the CP parity matrix $Q_{\nu}$. Freely varying the parameters $\theta_{l, \nu}$ and requiring the resulting mixing angles to be within the experimentally preferred ranges, we obtain the most general allowed regions of $m_{e e}$ versus the lightest neutrino mass $m_{\min }\left(m_{\min }=m_{1}\right.$ for $\mathrm{NH}$ and $m_{\min }=m_{3}$ for $\left.\mathrm{IH}\right)$, as shown in figure 3 . If the neutrino mass spectrum is $\mathrm{IH}$, we find that $m_{e e}$ is around $0.020 \mathrm{eV}$ or $0.048 \mathrm{eV}$ which are accessible to future $(\beta \beta)_{0 \nu}$-decay experiments. In the case of $\mathrm{NH}$, strong cancellation between different terms can occur for the CP parity $k_{1}=-1$ and $\varphi_{2}=\pi / 4$ such that $m_{e e}$ is smaller than $10^{-4} \mathrm{eV}$ for certain values of $m_{\min }$, while $m_{e e}$ is always larger than $1.219 \times 10^{-3} \mathrm{eV}$ for $\varphi_{2}=\pi / 2$.

(II) $Z_{2}^{g_{l}}=Z_{2}^{b c^{x} d^{x}}, X_{l}=\left\{c^{\gamma} d^{-2 x-\gamma}, b c^{x+\gamma} d^{-x-\gamma}\right\}, Z_{2}^{g_{\nu}}=Z_{2}^{a b c^{y}}, X_{\nu}=$ $\left\{\boldsymbol{c}^{\delta} \boldsymbol{d}^{2 \boldsymbol{2}+2 \delta}, \boldsymbol{a b} \boldsymbol{c}^{\boldsymbol{y}+\boldsymbol{\delta}} \boldsymbol{d}^{2 \boldsymbol{y}+2 \delta}\right\}$. In this case, the $\Sigma$ matrix is found to be

$$
\Sigma=\frac{1}{2}\left(\begin{array}{ccc}
1 & -\sqrt{2} e^{i \varphi_{4}} & -1 \\
-1 & -\sqrt{2} e^{i \varphi_{4}} & 1 \\
-\sqrt{2} e^{i \varphi_{3}} & 0 & -\sqrt{2} e^{i \varphi_{3}}
\end{array}\right),
$$

where an overall phase is omitted, and the parameters $\varphi_{3}$ and $\varphi_{4}$ determined by the remnant symmetries are of the form

$$
\varphi_{3}=\frac{3 \gamma+2(x+y)}{n} \pi, \quad \varphi_{4}=-\frac{3 \delta+2(x+y)}{n} \pi .
$$

We find that $\varphi_{3}$ and $\varphi_{4}$ are not completely independent of each other, and they can take the following discrete values,

$$
\begin{aligned}
& \varphi_{3}(\bmod 2 \pi)=0, \frac{1}{n} \pi, \frac{2}{n} \pi, \ldots, \frac{2 n-1}{n} \pi, \\
& \varphi_{3}+\varphi_{4}(\bmod 2 \pi)=0, \frac{3}{n} \pi, \frac{6}{n} \pi, \ldots, \frac{2 n-3}{n} \pi, \quad 3 \mid n, \\
& \varphi_{3}+\varphi_{4}(\bmod 2 \pi)=0, \frac{1}{n} \pi, \frac{2}{n} \pi, \ldots, \frac{2 n-1}{n} \pi, \quad 3 \nmid n .
\end{aligned}
$$



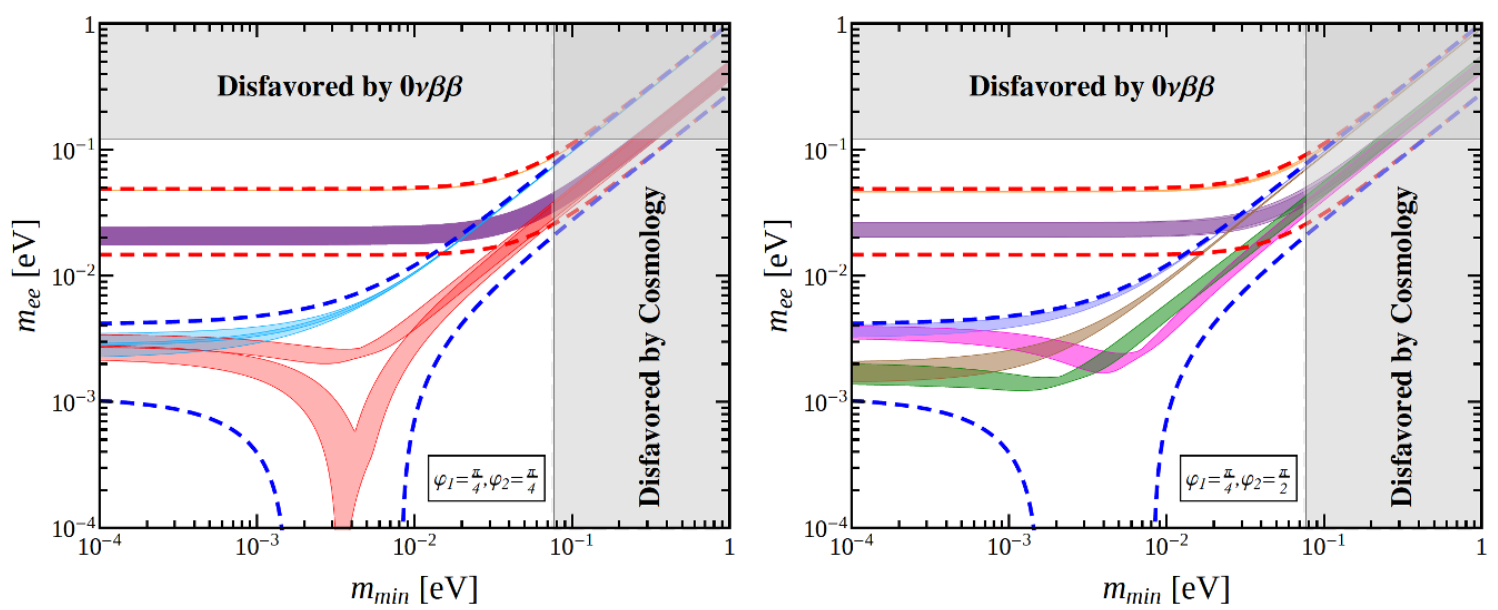

$\mathrm{IH}:\left(\mathrm{k}_{1}, \mathrm{k}_{2}\right)=(1,1),(1,-1) \quad \mathrm{IH}:\left(\mathrm{k}_{1}, \mathrm{k}_{2}\right)=(-1,1),(-1,-1) \quad \mathrm{NH}:\left(\mathrm{k}_{1}, \mathrm{k}_{2}\right)=(1,1) \quad \mathrm{NH}:\left(\mathrm{k}_{1}, \mathrm{k}_{2}\right)=(1,-1)$

$\mathrm{NH}:\left(\mathrm{k}_{1}, \mathrm{k}_{2}\right)=(-1,1) \quad \mathrm{NH}:\left(\mathrm{k}_{1}, \mathrm{k}_{2}\right)=(-1,-1) \quad \mathrm{NH}:\left(\mathrm{k}_{1}, \mathrm{k}_{2}\right)=(1,1),(1,-1) \quad \mathrm{NH}:\left(\mathrm{k}_{1}, \mathrm{k}_{2}\right)=(-1,1),(-1,-1)$

Figure 3. The possible values of the effective Majorana mass $m_{e e}$ as a function of the lightest neutrino mass $m_{\min }$ for the mixing pattern $U_{\mathrm{I}, 6}$ with $n=4$. The red (blue) dashed lines indicate the most general allowed regions for IH $(\mathrm{NH})$ neutrino mass spectrum obtained by varying the mixing parameters over their $3 \sigma$ ranges [8]. The present most stringent upper limits $m_{e e}<0.120 \mathrm{eV}$ from EXO-200 [85, 86] and KamLAND-ZEN [87] is shown by horizontal grey band. The vertical grey exclusion band denotes the current bound coming from the cosmological data of $\sum m_{i}<0.230 \mathrm{eV}$ at $95 \%$ confidence level obtained by the Planck collaboration [88].

Using the master formula of eq. (2.12), we obtain that the lepton mixing matrix is given by

$U_{\mathrm{II}}=\frac{1}{2}\left(\begin{array}{ccc}1 & c_{\nu}+\sqrt{2} e^{i \varphi_{4}} s_{\nu} & s_{\nu}-\sqrt{2} e^{i \varphi_{4}} c_{\nu} \\ s_{l}+\sqrt{2} e^{i \varphi_{3}} c_{l} & s_{l} c_{\nu}-\sqrt{2}\left(e^{i \varphi_{3}} c_{l} c_{\nu}+e^{i \varphi_{4}} s_{l} s_{\nu}\right) & s_{l} s_{\nu}-\sqrt{2}\left(e^{i \varphi_{3}} c_{l} s_{\nu}-e^{i \varphi_{4}} s_{l} c_{\nu}\right) \\ c_{l}-\sqrt{2} e^{i \varphi_{3}} s_{l} & c_{l} c_{\nu}+\sqrt{2}\left(e^{i \varphi_{3}} s_{l} c_{\nu}-e^{i \varphi_{4}} c_{l} s_{\nu}\right) & c_{l} s_{\nu}+\sqrt{2}\left(e^{i \varphi_{3}} s_{l} s_{\nu}+e^{i \varphi_{4}} c_{l} c_{\nu}\right)\end{array}\right)$.

It is easy to check that $U_{\text {II }}$ has the following symmetry transformations,

$$
\begin{aligned}
& U_{\mathrm{II}}\left(\varphi_{3}+\pi, \varphi_{4}, \theta_{l}, \theta_{\nu}\right)=\operatorname{diag}(1,1,-1) U_{\mathrm{II}}\left(\varphi_{3}, \varphi_{4}, \pi-\theta_{l}, \theta_{\nu}\right), \\
& U_{\mathrm{II}}\left(\varphi_{3}, \varphi_{4}+\pi, \theta_{l}, \theta_{\nu}\right)=U_{\mathrm{II}}\left(\varphi_{3}, \varphi_{4}, \theta_{l}, \pi-\theta_{\nu}\right) \operatorname{diag}(1,-1,1) .
\end{aligned}
$$

As a result, it is sufficient to focus on the fundamental intervals of $0 \leq \varphi_{3}<\pi$ and $0 \leq \varphi_{4}<\pi$. From eq. (3.30) we see that one element of the PMNS mixing matrix equals $1 / 2$ in this case. In order to be in accordance with experimental data, this fixed element $1 / 2$ can be identified with the $(21),(22),(31)$ or (32) entries of the mixing matrix. As a consequence, the PMNS mixing matrix can take the following four possible forms:

$$
\begin{aligned}
& U_{\mathrm{II}, 1}=P_{12} U_{\mathrm{II}}, \quad U_{\mathrm{II}, 2}=P_{12} U_{\mathrm{II}} P_{12}, \\
& U_{\mathrm{II}, 3}=P_{23} P_{12} U_{\mathrm{II}}, \quad U_{\mathrm{II}, 4}=P_{23} P_{12} U_{\mathrm{II}} P_{12} .
\end{aligned}
$$

We see that $U_{\mathrm{II}, 3}$ and $U_{\mathrm{II}, 4}$ are related to $U_{\mathrm{II}, 1}$ and $U_{\mathrm{II}, 2}$ through an exchange of the second and third rows of the PMNS mixing matrix, respectively. Thus the predictions of the PMNS 
matrices $U_{\mathrm{II}, 3}$ and $U_{\mathrm{II}, 4}$ for mixing angles and $\mathrm{CP}$ phases can be easily obtained from the results of $U_{\mathrm{II}, 1}$ and $U_{\mathrm{II}, 2}$, respectively. Subsequently we can read out the expressions of the mixing angles $\sin ^{2} \theta_{13}, \sin ^{2} \theta_{12}, \sin ^{2} \theta_{23}$ and the CP invariants $J_{\mathrm{CP}}, I_{1}, I_{2}$ for the mixing patterns $U_{\mathrm{II}, 1}$ and $U_{\mathrm{II}, 2}$. The results are summarized in table 5 . Since all the mixing parameters depend on the free parameters $\theta_{l, \nu}$, they are strongly correlated with each other. In particular, a sum rule among mixing angles and Dirac CP phase can be found for each mixing pattern,

$$
\begin{aligned}
& U_{\mathrm{II}, 1}: \quad \cos \delta_{\mathrm{CP}}=\frac{1-4 \sin ^{2} \theta_{12} \cos ^{2} \theta_{23}-4 \sin ^{2} \theta_{13} \cos ^{2} \theta_{12} \sin ^{2} \theta_{23}}{2 \sin 2 \theta_{12} \sin \theta_{13} \sin 2 \theta_{23}}, \\
& U_{\mathrm{II}, 2}: \quad \cos \delta_{\mathrm{CP}}=-\frac{1-4 \cos ^{2} \theta_{12} \cos ^{2} \theta_{23}-4 \sin ^{2} \theta_{13} \sin ^{2} \theta_{12} \sin ^{2} \theta_{23}}{2 \sin 2 \theta_{12} \sin \theta_{13} \sin 2 \theta_{23}}, \\
& U_{\mathrm{II}, 3}: \quad \cos \delta_{\mathrm{CP}}=-\frac{1-4 \sin ^{2} \theta_{12} \sin ^{2} \theta_{23}-4 \sin ^{2} \theta_{13} \cos ^{2} \theta_{12} \cos ^{2} \theta_{23}}{2 \sin 2 \theta_{12} \sin \theta_{13} \sin 2 \theta_{23}}, \\
& U_{\mathrm{II}, 4}: \quad \cos \delta_{\mathrm{CP}}=\frac{1-4 \cos ^{2} \theta_{12} \sin ^{2} \theta_{23}-4 \sin ^{2} \theta_{13} \sin ^{2} \theta_{12} \cos ^{2} \theta_{23}}{2 \sin 2 \theta_{12} \sin \theta_{13} \sin 2 \theta_{23}} .
\end{aligned}
$$

The above sum rules for $\cos \delta_{\mathrm{CP}}$ in terms of mixing angles are also derived in ref. [63]. The reason is that these relations are obtained from the (21), (22), (31) or (32) entries of the PMNS matrix equal to $1 / 2$, respectively. Since the lepton mixing matrix depends on two free parameters in our model and three free parameters in [63], the correlation between the neutrino mixing parameters, as well as their variation ranges are different in these two work. Hence the predictions for $\cos \delta_{\mathrm{CP}}$ are not the same as those of ref. [63]. If all the three mixing angles freely vary within their $3 \sigma$ intervals [8], the above four relations lead to $\cos \delta_{\mathrm{CP}} \in$ $[0.444,1],[-0.105,1],[-1,-0.356]$ and $[-1,-0.0976]$, respectively. The different regions of $\cos \delta_{\mathrm{CP}}$ allow us to distinguish the four mixing patterns. We see that only the mixing matrix $U_{\mathrm{II}, 2}$ can accommodate a maximal Dirac CP phase $\delta_{\mathrm{CP}}=3 \pi / 2$. Hence the other three mixing matrices would be ruled out if the signal of maximal $\delta_{\mathrm{CP}}$ is confirmed by future neutrino facilities. If both $\theta_{12}$ and $\theta_{23}$ are measured more precisely and their experimental errors are reduced considerably in future, one could use these relations to predict the Dirac CP phase $\delta_{\mathrm{CP}}$ from the experimental values of the mixing angles. The above mixing sum rules are quite sensitive probes to test this type of mixing pattern [89-94]. In principle we can express $\theta_{l}$ and $\theta_{\nu}$ in terms of any two mixing angles, thus four sum rules among the mixing angles and $\mathrm{CP}$ phases can be obtained for each mixing pattern. However, the resulting sum rules except those in eq. (3.33) are usually rather long, very complex and not very illuminating, hence we don't show these results. Furthermore, as shown in ref. [60], the simple $S_{4}$ flavor group can already accommodate the measured values of the lepton mixing angles for $\left(\varphi_{3}, \varphi_{4}\right)=(0,0),(0, \pi / 2)$ and $(\pi / 2,0)$ which correspond to $\left(X_{l}, X_{\nu}\right)=(U, T)$, $(U, S T S)$ and $\left(T^{2}, T\right)$ with $\left(G_{l}, G_{\nu}\right)=\left(Z_{2}^{S T^{2} S U}, Z_{2}^{T U}\right)$ in the notation of [60]. For the next small group index $n=3$, there are only two independent cases corresponding to $\left(\varphi_{3}, \varphi_{4}\right)=$ $(0,0),(\pi / 3,2 \pi / 3)$. We find that the mixing pattern for $\left(\varphi_{3}, \varphi_{4}\right)=(0,0)$ is equivalent to that of case I with $\left(\varphi_{1}, \varphi_{2}\right)=(\pi / 3,0)$, the same predictions for mixing angles and CP phases are obtained. For the second case $\left(\varphi_{3}, \varphi_{4}\right)=(\pi / 3,2 \pi / 3)$, detailed numerical analyses show that all the three mixing angles can not simultaneously lie in their respective $3 \sigma$ ranges for 


\begin{tabular}{|c|c|}
\hline \multicolumn{2}{|r|}{ Case II } \\
\hline \multirow{5}{*}{$U_{\mathrm{II}, 1}$} & $\sin ^{2} \theta_{13}=\frac{s_{\nu}^{2}+c_{l}^{2} s_{\nu}^{2}+2 c_{\nu}^{2} s_{l}^{2}}{4}+\frac{s_{l} s_{\nu}\left(c_{\nu} s_{l} \cos \varphi_{4}-c_{l} s_{\nu} \cos \varphi_{3}-\sqrt{2} c_{l} c_{\nu} \cos \left(\varphi_{3}-\varphi_{4}\right)\right)}{\sqrt{2}}$ \\
\hline & $\sin ^{2} \theta_{12}=\frac{2 c_{l}^{2} c_{\nu}^{2}-2 \sqrt{2} c_{\nu} s_{l}\left(c_{l} c_{\nu} \cos \varphi_{3}-\sqrt{2} c_{l} s_{\nu} \cos \left(\varphi_{3}-\varphi_{4}\right)+s_{l} s_{\nu} \cos \varphi_{4}\right)+c_{\nu}^{2} s_{l}^{2}+2 s_{l}^{2} s_{\nu}^{2}}{4-\left(s_{\nu}^{2}+c_{l}^{2} s_{\nu}^{2}+2 c_{\nu}^{2} s_{l}^{2}\right)-2 \sqrt{2} s_{l} s_{\nu}\left(c_{\nu} s_{l} \cos \varphi_{4}-c_{l} s_{\nu} \cos \varphi_{3}-\sqrt{2} c_{l} c_{\nu} \cos \left(\varphi_{3}-\varphi_{4}\right)\right)}$ \\
\hline & $\sin ^{2} \theta_{23}=\frac{1+c_{\nu}^{2}-2 \sqrt{2} c_{\nu} s_{\nu} \cos \varphi_{4}}{4-\left(s_{\nu}^{2}+c_{l}^{2} s_{\nu}^{2}+2 c_{\nu}^{2} s_{l}^{2}\right)-2 \sqrt{2} s_{l} s_{\nu}\left(c_{\nu} s_{l} \cos \varphi_{4}-c_{l} s_{\nu} \cos \varphi_{3}-\sqrt{2} c_{l} c_{\nu} \cos \left(\varphi_{3}-\varphi_{4}\right)\right)}$ \\
\hline & $\begin{aligned}\left|I_{1}\right|= & \mid \frac{1}{8} s_{l}\left[2 c_{l} s_{\nu} \sin \left(\varphi_{3}-\varphi_{4}\right)-\sqrt{2}\left(2 c_{l} c_{\nu} \sin \varphi_{3}+s_{l} s_{\nu} \sin \varphi_{4}\right)\right] \\
& \times\left[c_{\nu}\left(1-3 c_{l}^{2}\right)-s_{l} s_{\nu}\left(2 c_{l} \cos \left(\varphi_{3}-\varphi_{4}\right)-\sqrt{2} s_{l} \cos \varphi_{4}\right)\right] \mid\end{aligned}$ \\
\hline & $\begin{aligned}\left|I_{2}\right|= & \mid \frac{1}{8} s_{l}\left[2 c_{l} c_{\nu} \sin \left(\varphi_{3}-\varphi_{4}\right)-\sqrt{2}\left(c_{\nu} s_{l} \sin \varphi_{4}-2 c_{l} s_{\nu} \sin \varphi_{3}\right)\right] \\
& \times\left[s_{\nu}\left(1-3 c_{l}^{2}\right)+s_{l} c_{\nu}\left(2 c_{l} \cos \left(\varphi_{3}-\varphi_{4}\right)+\sqrt{2} s_{l} \cos \varphi_{4}\right)\right]\end{aligned}$ \\
\hline \multirow{5}{*}{$U_{\mathrm{II}, 2}$} & $\sin ^{2} \theta_{13}=\frac{s_{\nu}^{2}+c_{l}^{2} s_{\nu}^{2}+2 c_{\nu}^{2} s_{l}^{2}}{4}+\frac{s_{l} s_{\nu}\left(c_{\nu} s_{l} \cos \varphi_{4}-c_{l} s_{\nu} \cos \varphi_{3}-\sqrt{2} c_{l} c_{\nu} \cos \left(\varphi_{3}-\varphi_{4}\right)\right)}{\sqrt{2}}$ \\
\hline & $\sin ^{2} \theta_{12}=\frac{1+c_{l}^{2}+2 \sqrt{2} s_{l} c_{l} \cos \varphi_{3}}{4-\left(s_{\nu}^{2}+c_{l}^{2} s_{\nu}^{2}+2 c_{\nu}^{2} s_{l}^{2}\right)-2 \sqrt{2} s_{l} s_{\nu}\left(c_{\nu} s_{l} \cos \varphi_{4}-c_{l} s_{\nu} \cos \varphi_{3}-\sqrt{2} c_{l} c_{\nu} \cos \left(\varphi_{3}-\varphi_{4}\right)\right)}$ \\
\hline & $\sin ^{2} \theta_{23}=\frac{c_{\nu}^{2}-2 \sqrt{2} c_{\nu} s_{\nu} \cos \varphi_{4}+1}{4-\left(s_{\nu}^{2}+c_{l}^{2} s_{\nu}^{2}+2 c_{\nu}^{2} s_{l}^{2}\right)-2 \sqrt{2} s_{l} s_{\nu}\left(c_{\nu} s_{l} \cos \varphi_{4}-c_{l} s_{\nu} \cos \varphi_{3}-\sqrt{2} c_{l} c_{\nu} \cos \left(\varphi_{3}-\varphi_{4}\right)\right)}$ \\
\hline & $\begin{aligned}\left|I_{1}\right|= & \mid \frac{1}{8} s_{l}\left[2 c_{l} s_{\nu} \sin \left(\varphi_{3}-\varphi_{4}\right)-\sqrt{2}\left(2 c_{l} c_{\nu} \sin \varphi_{3}+s_{l} s_{\nu} \sin \varphi_{4}\right)\right] \\
& \times\left[c_{\nu}\left(1-3 c_{l}^{2}\right)-s_{l} s_{\nu}\left(2 c_{l} \cos \left(\varphi_{3}-\varphi_{4}\right)-\sqrt{2} s_{l} \cos \varphi_{4}\right)\right]\end{aligned}$ \\
\hline & $\begin{aligned}\left|I_{2}\right|= & \mid \frac{1}{8} s_{l}\left[\sqrt{2}\left(2 c_{l} c_{\nu} \sin \varphi_{3}+s_{l} s_{\nu} \sin \varphi_{4}\right)-2 c_{l} s_{\nu} \sin \left(\varphi_{3}-\varphi_{4}\right)\right] \\
& \times\left[c_{\nu}\left(1-3 c_{l}^{2}\right)-s_{l} s_{\nu}\left(\sqrt{2} s_{l} \cos \varphi_{4}+2 c_{l} \cos \left(\varphi_{3}-\varphi_{4}\right)\right)\right]\end{aligned}$ \\
\hline
\end{tabular}

Table 5. The predictions for the mixing parameters in the case II. For all the two mixing patterns $U_{\mathrm{II}, 1}$ and $U_{\mathrm{II}, 2}$, the absolute value of the Jarlskog invariant $J_{\mathrm{CP}}$ is the same with $\left|J_{\mathrm{CP}}\right|=\frac{1}{8}\left|\sqrt{2} c_{\nu} s_{\nu}\left(s_{l}^{2}-c_{l}^{2}\right) \sin \varphi_{4}+\sqrt{2} c_{l} s_{l}\left(c_{\nu}^{2}-s_{\nu}^{2}\right) \sin \varphi_{3}+c_{l} c_{\nu} s_{l} s_{\nu} \sin \left(\varphi_{3}+\varphi_{4}\right)\right|$.

any values of $\theta_{l, \nu}$, consequently agreement with the data can not be achieved. In order to obtain new mixing patterns with non-trivial $\mathrm{CP}$ phases, we will discuss the flavor group $\Delta\left(6 \cdot 4^{2}\right)=\Delta(96)$. The phenomenologically viable values of $\varphi_{3}$ and $\varphi_{4}$ are $\left(\varphi_{3}, \varphi_{4}\right)=(0,0)$, $(0, \pi / 4),(0,3 \pi / 4),(\pi / 4, \pi / 2),(3 \pi / 4, \pi / 2)$ for mixing matrix $U_{\mathrm{II}, 1}$ and $\left(\varphi_{3}, \varphi_{4}\right)=(0,0)$, $(0, \pi / 4),(0,3 \pi / 4),(\pi / 4,0),(\pi / 4, \pi / 4),(\pi / 2, \pi / 4),(\pi / 2,3 \pi / 4),(3 \pi / 4,0),(3 \pi / 4,3 \pi / 4)$ for mixing matrix $U_{\mathrm{II}, 2}$. We notice that the resulting mixing matrices for $\left(\varphi_{3}, \varphi_{4}\right)=$ $(0,3 \pi / 4),(3 \pi / 4, \pi / 2),(\pi / 2,3 \pi / 4),(3 \pi / 4,0),(3 \pi / 4,3 \pi / 4)$ are the complex conjugate of those for $\left(\varphi_{3}, \varphi_{4}\right)=(0, \pi / 4),(\pi / 4, \pi / 2),(\pi / 2, \pi / 4),(\pi / 4,0),(\pi / 4, \pi / 4)$ respectively up to redefinition of $\theta_{\nu}, \theta_{l}, Q_{l}$ and $Q_{\nu}$. Two mixing matrices conjugate to each other would not be regarded as independent since we are concerned with the absolute values of sines of the $\mathrm{CP}$ violation phases. In the case of $\varphi_{3}=\varphi_{4}=0$, all the three $\mathrm{CP}$ phases are trivial because an accidental CP transformation $X=c^{\gamma} d^{-2 x-\gamma}$ is present in both the 

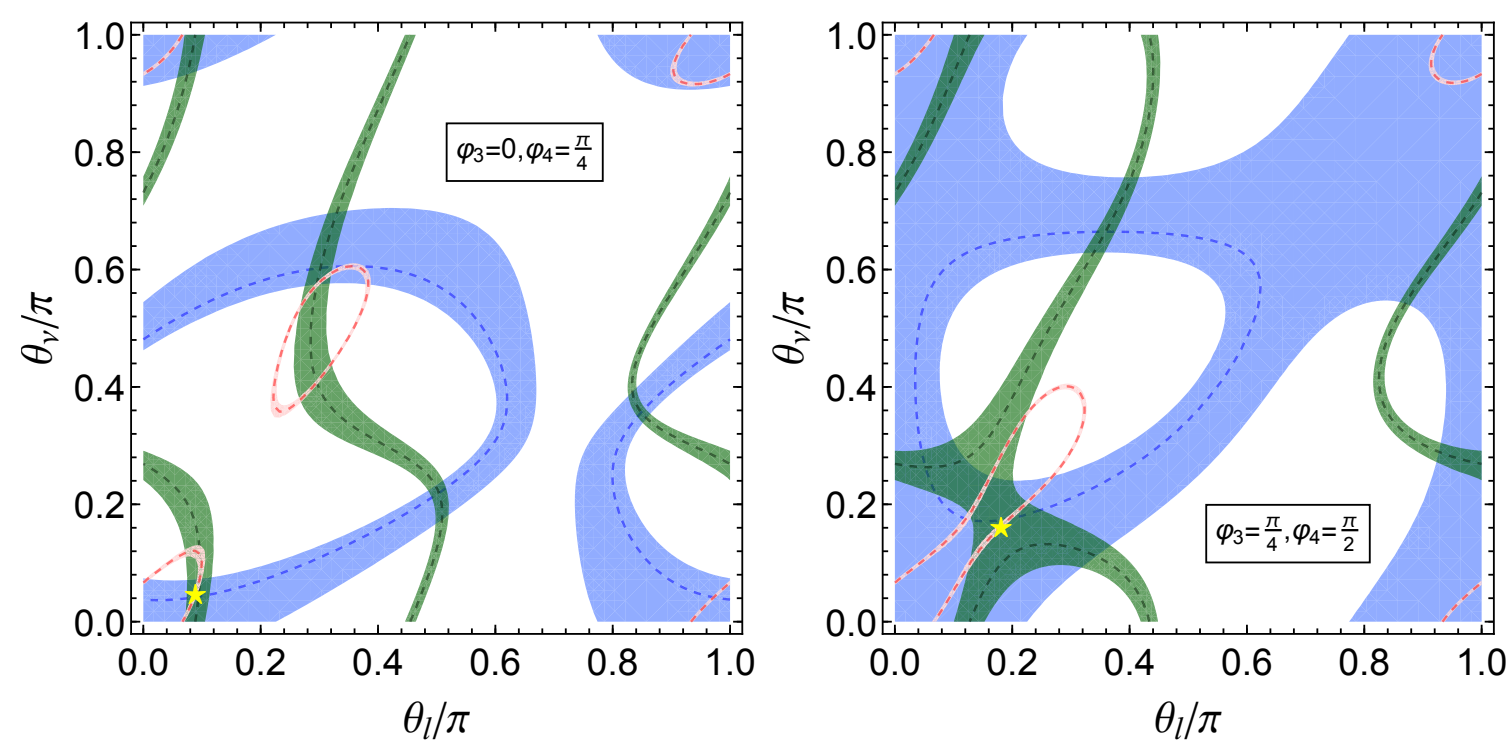

Figure 4. Contour plots of $\sin ^{2} \theta_{i j}$ in the $\theta_{\nu}-\theta_{l}$ plane for $U_{\mathrm{II}, 1}$ with $n=4$. The red, green and blue areas denote the $3 \sigma$ contour regions of $\sin ^{2} \theta_{13}, \sin ^{2} \theta_{12}$ and $\sin ^{2} \theta_{23}$ respectively. The dashed contour lines represent the corresponding experimental best fit values. The $3 \sigma$ ranges as well as the best fit values of the mixing angles are adapted from [8]. The best fitting values of $\theta_{l, \nu}$ are indicated with yellow pentagrams.

neutrino and charged lepton sectors. For the above representative values of $\varphi_{3}$ and $\varphi_{4}$, the variations of $\sin ^{2} \theta_{12}, \sin ^{2} \theta_{13}$ and $\sin ^{2} \theta_{23}$ in the plane $\theta_{\nu}$ versus $\theta_{l}$ are displayed in figure 4 and figure 5 for $U_{\mathrm{II}, 1}$ and $U_{\mathrm{II}, 2}$, respectively. The results of the $\chi^{2}$ analysis are summarized in table 7 . We display the contour plots of $\left|\sin \delta_{\mathrm{CP}}\right|,\left|\sin \alpha_{21}\right|$ and $\left|\sin \alpha_{31}\right|$ in figure 6 and figure 7 for the mixing patterns $U_{\mathrm{II}, 1}$ and $U_{\mathrm{II}, 2}$ respectively. Freely varying the parameters $\theta_{l, \nu}$ and requiring all the three mixing angles are within their $3 \sigma$ ranges, the allowed intervals of $\left|\sin \delta_{\mathrm{CP}}\right|,\left|\sin \alpha_{21}\right|$ and $\left|\sin \alpha_{31}\right|$ are collected in table 6 . We see that the mixing matrices $U_{\mathrm{II}, 1}$ with $\left(\varphi_{3}, \varphi_{4}\right)=(0, \pi / 4),(\pi / 4, \pi / 2)$ and $U_{\mathrm{II}, 2}$ with $\varphi_{3}=\varphi_{4}=\pi / 4$ can not accommodate a nearly maximal Dirac CP phase. Examining the numerical results in table 7 and table 6 , we find that the predictions for $\theta_{13}$ are almost the same, nevertheless $\theta_{12}, \theta_{23}$ and $\delta_{\mathrm{CP}}$ are predicted to be distinct from each other although the differences are not so large. The upcoming reactor neutrino oscillation experiments, such as JUNO [95] and RENO-50 [96], expect to make very precise measurements of the solar neutrino mixing angle $\theta_{12}$. They will be capable of reducing the error of $\theta_{12}$ to about $0.1^{\circ}$ or around $0.3 \%$. Future long baseline experiments DUNE [76-79], T2HK [80, 81], T2HKK [82] can make very precise measurements of the oscillation parameters $\theta_{12}, \theta_{23}$ and $\delta_{\mathrm{CP}}$. Therefore future neutrino facilities have the potential to discriminate between the above possible cases, or to rule them out entirely. Furthermore, we expect that a more ambitious facility such as the neutrino factory [97-99] could provide a more stringent tests of our approach. Furthermore, we can read out the effective Majorana neutrino mass as

$$
\begin{aligned}
m_{e e}= & \frac{1}{4} \mid\left(s_{l}+\sqrt{2} e^{i \varphi_{3}} c_{l}\right)^{2} m_{1}+k_{1}\left[s_{l} c_{\nu}-\sqrt{2}\left(e^{i \varphi_{3}} c_{l} c_{\nu}+e^{i \varphi_{4}} s_{l} s_{\nu}\right)\right]^{2} m_{2} \\
& +k_{2}\left[s_{l} s_{\nu}-\sqrt{2}\left(e^{i \varphi_{3}} c_{l} s_{\nu}-e^{i \varphi_{4}} s_{l} c_{\nu}\right)\right]^{2} m_{3} \mid,
\end{aligned}
$$




\begin{tabular}{|c|c|c|c|c|c|}
\hline & $\varphi_{3}$ & $\varphi_{4}$ & $\left|\sin \delta_{\mathrm{CP}}\right|$ & $\left|\sin \alpha_{21}\right|$ & $\left|\sin \alpha_{31}\right|$ \\
\hline \multirow{2}{*}{$U_{\mathrm{II}, 1}$} & 0 & $\frac{\pi}{4}$ & {$[0.00312,0.774]$} & {$[0.000450,0.131] \cup[0.999,1]$} & {$[0.210,0.223] \cup[0.995,1]$} \\
\cline { 2 - 6 } & $\frac{\pi}{4}$ & $\frac{\pi}{2}$ & {$[0.067,0.822]$} & {$[0.820,1]$} & {$[0.492,0.939]$} \\
\hline \multirow{4}{*}{$U_{\mathrm{II}, 2}$} & 0 & $\frac{\pi}{4}$ & {$[0.00227,0.997]$} & {$[0.000169,0.0869]$} & {$[0.997,1]$} \\
\cline { 2 - 6 } & $\frac{\pi}{4}$ & 0 & {$[0.100,0.607] \cup[0.911,1]$} & {$[0.0695,0.355] \cup[0.613,0.749]$} & {$[0.0452,0.215] \cup[0.396,0.542]$} \\
\cline { 2 - 6 } & $\frac{\pi}{4}$ & $\frac{\pi}{4}$ & {$[0.647,0.711]$} & {$[0.578,0.806]$} & {$[0.823,0.935]$} \\
\cline { 2 - 6 } & $\frac{\pi}{2}$ & $\frac{\pi}{4}$ & {$[0,0.998]$} & {$[0,0.859]$} & {$[0.819,1]$} \\
\hline
\end{tabular}

Table 6. The allowed regions of $\left|\sin \delta_{\mathrm{CP}}\right|, \sin \alpha_{21}$ and $\left|\sin \alpha_{31}\right|$ for the viable mixing patterns of case II with $n=4$, where both free parameters $\theta_{l}$ and $\theta_{\nu}$ freely vary in the fundamental region $[0, \pi]$ and all the three lepton mixing angles are required to lie within their $3 \sigma$ intervals [8].

\begin{tabular}{|c|c|c|c|c|c|c|c|c|c|c|c|}
\hline \multicolumn{12}{|c|}{ Case II with $n=4$} \\
\hline & $\varphi_{3}$ & $\varphi_{4}$ & $\theta_{l}^{\mathrm{bf}} / \pi$ & $\theta_{\nu}^{\mathrm{bf}} / \pi$ & $\chi_{\min }^{2}$ & $\sin ^{2} \theta_{13}$ & $\sin ^{2} \theta_{12}$ & $\sin ^{2} \theta_{23}$ & $\left|\sin \delta_{\mathrm{CP}}\right|$ & $\left|\sin \alpha_{21}\right|$ & $\left|\sin \alpha_{31}\right|$ \\
\hline \multirow{4}{*}{$U_{\mathrm{II}, 1}$} & 0 & $\underline{\pi}$ & 0.0889 & 0.047 & 1.048 & 0.0218 & 0.317 & 0.431 & 0.361 & 0.0728 & 0.999 \\
\hline & 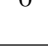 & $\overline{4}$ & $(0.0717)$ & $(0.00688)$ & $(23.492)$ & $(0.0223)$ & $(0.344)$ & $(0.500)$ & $(0.0581)$ & $(0.00833)$ & $(1.000)$ \\
\hline & $\pi$ & & 0.181 & 0.162 & 4.501 & 0.0217 & 0.331 & 0.451 & 0.451 & 1.000 & 0.780 \\
\hline & $\overline{4}$ & $\overline{2}$ & $(0.131)$ & $(0.102)$ & $(26.930)$ & $(0.0221)$ & $(0.342)$ & $(0.486)$ & $(0.307)$ & $(0.868)$ & $(0.535)$ \\
\hline \multirow{8}{*}{$U_{\mathrm{II}, 2}$} & 0 & & 0.921 & 0.984 & 14.578 & 0.0219 & 0.323 & 0.536 & 0.194 & 0.0152 & 1.000 \\
\hline & 0 & $\overline{4}$ & $(0.910)$ & $(0.966)$ & $(1.476)$ & $(0.0217)$ & $(0.299)$ & $(0.562)$ & $(0.417)$ & $(0.0355)$ & $(0.999)$ \\
\hline & & & 0.464 & 0.625 & 16.579 & 0.0219 & 0.316 & 0.548 & 0.399 & 0.252 & 0.156 \\
\hline & $\frac{1}{4}$ & 0 & $(0.472)$ & $(0.628)$ & $(1.834)$ & $(0.0217)$ & $(0.302)$ & $(0.556)$ & $(0.313)$ & $(0.201)$ & $(0.126)$ \\
\hline & & & 0.892 & 0.964 & 22.734 & 0.0218 & 0.322 & 0.565 & 0.694 & 0.654 & 0.908 \\
\hline & $\frac{\pi}{4}$ & $\frac{\pi}{4}$ & $(0.882)$ & $(0.956)$ & $(0.238)$ & $(0.0218)$ & $(0.304)$ & $(0.576)$ & $(0.685)$ & $(0.707)$ & $(0.884)$ \\
\hline & & & 0.643 & 0.677 & 17.979 & 0.0218 & 0.304 & 0.555 & 0.341 & 0.486 & 0.969 \\
\hline & $\overline{2}$ & $\overline{4}$ & $(0.650)$ & $(0.677)$ & $(1.677)$ & $(0.0217)$ & $(0.308)$ & $(0.556)$ & $(0.441)$ & $(0.423)$ & $(0.957)$ \\
\hline
\end{tabular}

Table 7. Results of the $\chi^{2}$ analysis for $n=4$ in case II. The $\chi^{2}$ function obtains a global minimum $\chi_{\min }^{2}$ at the best fit values $\left(\theta_{l}, \theta_{\nu}\right)=\left(\theta_{l}^{\mathrm{bf}}, \theta_{\nu}^{\mathrm{bf}}\right)$. We display the values of the mixing angles and CP violation phases at the best fitting point. The numbers given in parentheses are the corresponding results for the inverted hierarchy neutrino mass spectrum. Here we do not show the results of $\varphi_{3}=\varphi_{4}=0$ for which all the three CP phases are trivial.

for $U_{\mathrm{II}, 1}$ and

$$
\begin{aligned}
m_{e e}= & \frac{1}{4} \mid\left[s_{l} c_{\nu}-\sqrt{2}\left(e^{i \varphi_{3}} c_{l} c_{\nu}+e^{i \varphi_{4}} s_{l} s_{\nu}\right)\right]^{2} m_{1}+k_{1}\left(s_{l}+\sqrt{2} e^{i \varphi_{3}} c_{l}\right)^{2} m_{2} \\
& +k_{2}\left[s_{l} s_{\nu}-\sqrt{2}\left(e^{i \varphi_{3}} c_{l} s_{\nu}-e^{i \varphi_{4}} s_{l} c_{\nu}\right)\right]^{2} m_{3} \mid
\end{aligned}
$$

for $U_{\mathrm{II}, 2}$. The corresponding predictions for the $(\beta \beta)_{0 \nu}$-decay effective mass as a function of the lightest neutrino mass are shown in figure 8 and figure 9 for the mixing matrices $U_{\text {II, } 1}$ and $U_{\mathrm{II}, 2}$, respectively. It is remarkable that the effective mass $m_{e e}$ is always larger than $10^{-3} \mathrm{eV}$ for the mixing patterns $U_{\mathrm{II}, 1}$ with $\left(\varphi_{3}, \varphi_{4}\right)=(\pi / 4, \pi / 2)$ and $U_{\mathrm{II}, 2}$ with $\left(\varphi_{3}, \varphi_{4}\right)=$ $(\pi / 4, \pi / 4)$ in the case of $\mathrm{NH}$ spectrum, while for other mixing patterns $m_{e e}$ can be strongly suppressed due to cancellations for certain values of $m_{\min }$.

(III) $Z_{2}^{g_{l}}=Z_{2}^{b c^{x} d^{x}}, X_{l}=\left\{c^{\gamma} d^{-2 x-\gamma}, b c^{x+\gamma} d^{-x-\gamma}\right\}, Z_{2}^{g_{\nu}}=Z_{2}^{c^{n / 2}}, X_{\nu}=\left\{c^{\alpha} d^{\delta}\right\}$. In this case, the index $n$ has to be even in order to have a $Z_{2}$ subgroup generated by the 

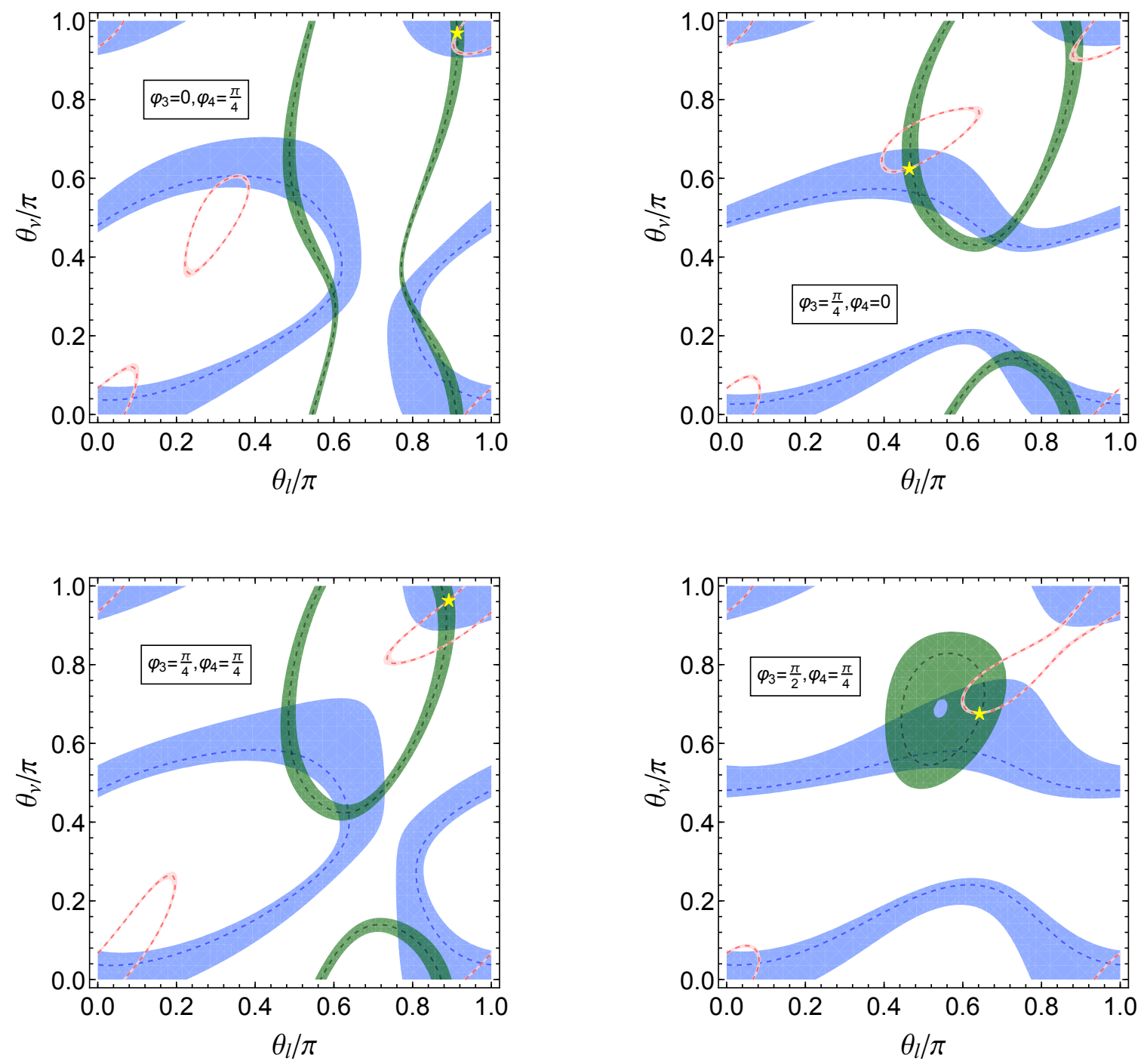

Figure 5. Contour plots of $\sin ^{2} \theta_{i j}$ in the $\theta_{\nu}-\theta_{l}$ plane for $U_{\mathrm{II}, 2}$ with $n=4$. The red, green and blue areas denote the $3 \sigma$ contour regions of $\sin ^{2} \theta_{13}, \sin ^{2} \theta_{12}$ and $\sin ^{2} \theta_{23}$ respectively. The dashed contour lines represent the corresponding experimental best fit values. The $3 \sigma$ ranges as well as the best fit values of the mixing angles are adapted from [8]. The best fitting values of $\theta_{l, \nu}$ are indicated with yellow pentagrams.

element $c^{n / 2}$. The parameters $x, \gamma, \alpha$ and $\delta$ can be any integer from 0 to $n-1$. We can read out the $\Sigma$ matrix as

$$
\Sigma=\frac{1}{\sqrt{2}}\left(\begin{array}{ccc}
e^{i \varphi_{6}} & 0 & -1 \\
e^{i \varphi_{6}} & 0 & 1 \\
0 & \sqrt{2} e^{i \varphi_{5}} & 0
\end{array}\right)
$$

with

$$
\varphi_{5}=\frac{2 x-2 \alpha+3 \gamma+\delta}{n} \pi, \quad \varphi_{6}=-\frac{2 x+\alpha+\delta}{n} \pi
$$



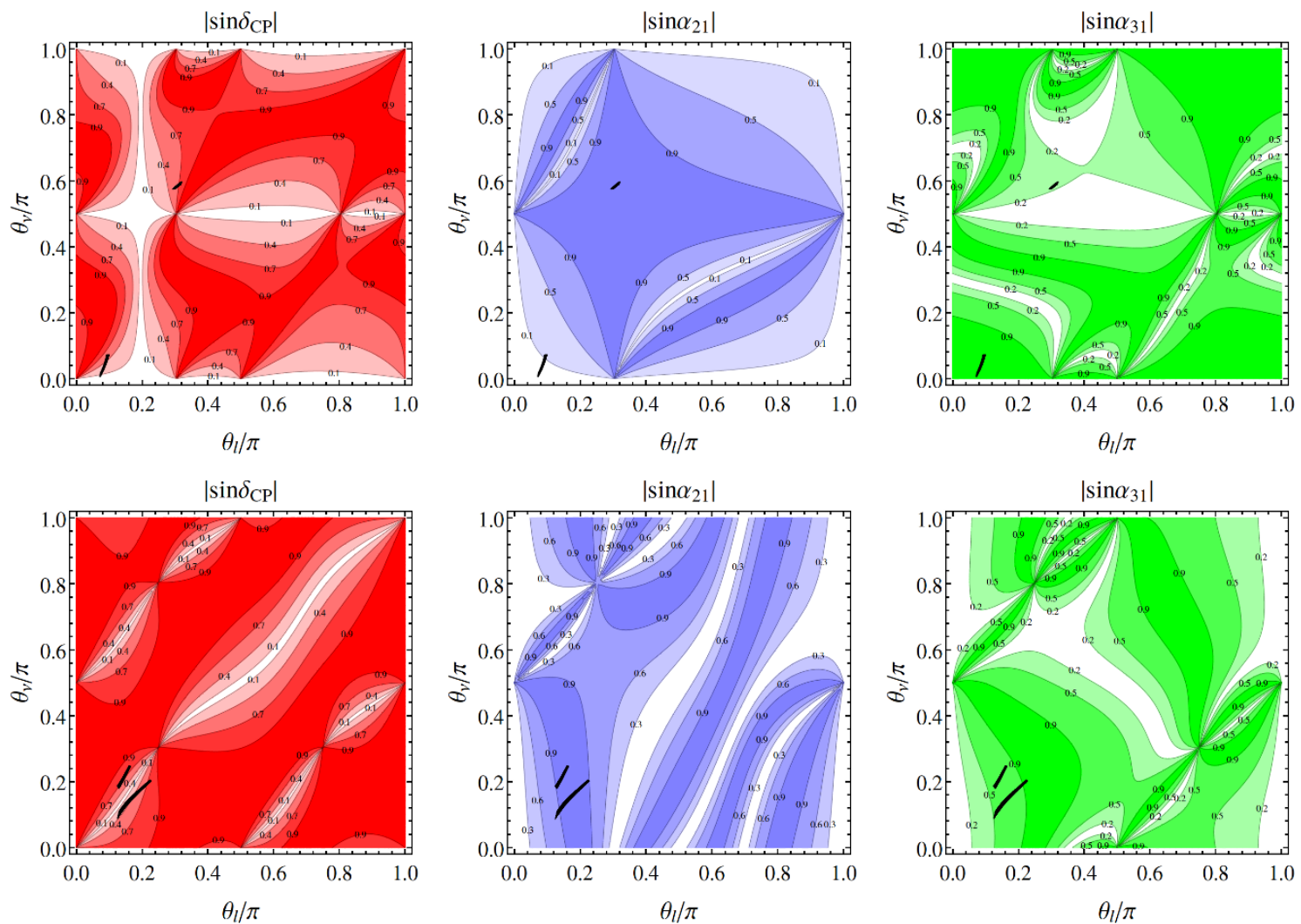

Figure 6. The contour plots of the $\mathrm{CP}$ violation phases $\left|\sin \delta_{\mathrm{CP}}\right|,\left|\sin \alpha_{21}\right|$ and $\left|\sin \alpha_{31}\right|$ for the mixing pattern $U_{\mathrm{II}, 1}$, where the parameters $\left(\varphi_{3}, \varphi_{4}\right)$ are equal to $(0, \pi / 4)$ in the upper panels and $(\pi / 4, \pi / 2)$ in the lower panels. The black areas represent the regions for which all the three lepton mixing angles lie in their corresponding experimentally allowed $3 \sigma$ intervals [8]. Since $U_{\mathrm{II}, 1}$ and $U_{\mathrm{II}, 3}$ are related through the exchange of the second and the third rows of the mixing matrix, they lead to the same Majorana phases $\alpha_{21}$ and $\alpha_{31}$ while the Dirac phase changes from $\delta_{\mathrm{CP}}$ to $\pi+\delta_{\mathrm{CP}}$.

We see that the discrete values of $\varphi_{5}$ and $\varphi_{6}$ are correlated in the case that $n$ is divisible by 3 . To be specific, their values could be

$$
\begin{aligned}
& \varphi_{5}(\bmod 2 \pi)=0, \frac{1}{n} \pi, \frac{2}{n} \pi, \ldots, \frac{2 n-1}{n} \pi, \\
& \varphi_{5}+\varphi_{6}(\bmod 2 \pi)=0, \frac{3}{n} \pi, \frac{6}{n} \pi, \ldots, \frac{2 n-3}{n} \pi, \quad 3 \mid n \\
& \varphi_{5}+\varphi_{6}(\bmod 2 \pi)=0, \frac{1}{n} \pi, \frac{2}{n} \pi, \ldots, \frac{2 n-1}{n} \pi, \quad 3 \nmid n .
\end{aligned}
$$

Using eq. (2.12), we find that the lepton mixing matrix takes the following form

$$
U_{\mathrm{III}}=\frac{1}{\sqrt{2}}\left(\begin{array}{ccc}
c_{\nu} & s_{\nu} & -e^{i \varphi_{6}} \\
s_{l} c_{\nu}+\sqrt{2} e^{i \varphi_{5}} c_{l} s_{\nu} & s_{l} s_{\nu}-\sqrt{2} e^{i \varphi_{5}} c_{l} c_{\nu} & e^{i \varphi_{6}} s_{l} \\
c_{l} c_{\nu}-\sqrt{2} e^{i \varphi_{5}} s_{l} s_{\nu} & c_{l} s_{\nu}+\sqrt{2} e^{i \varphi_{5}} s_{l} c_{\nu} & e^{i \varphi_{6}} c_{l}
\end{array}\right) .
$$



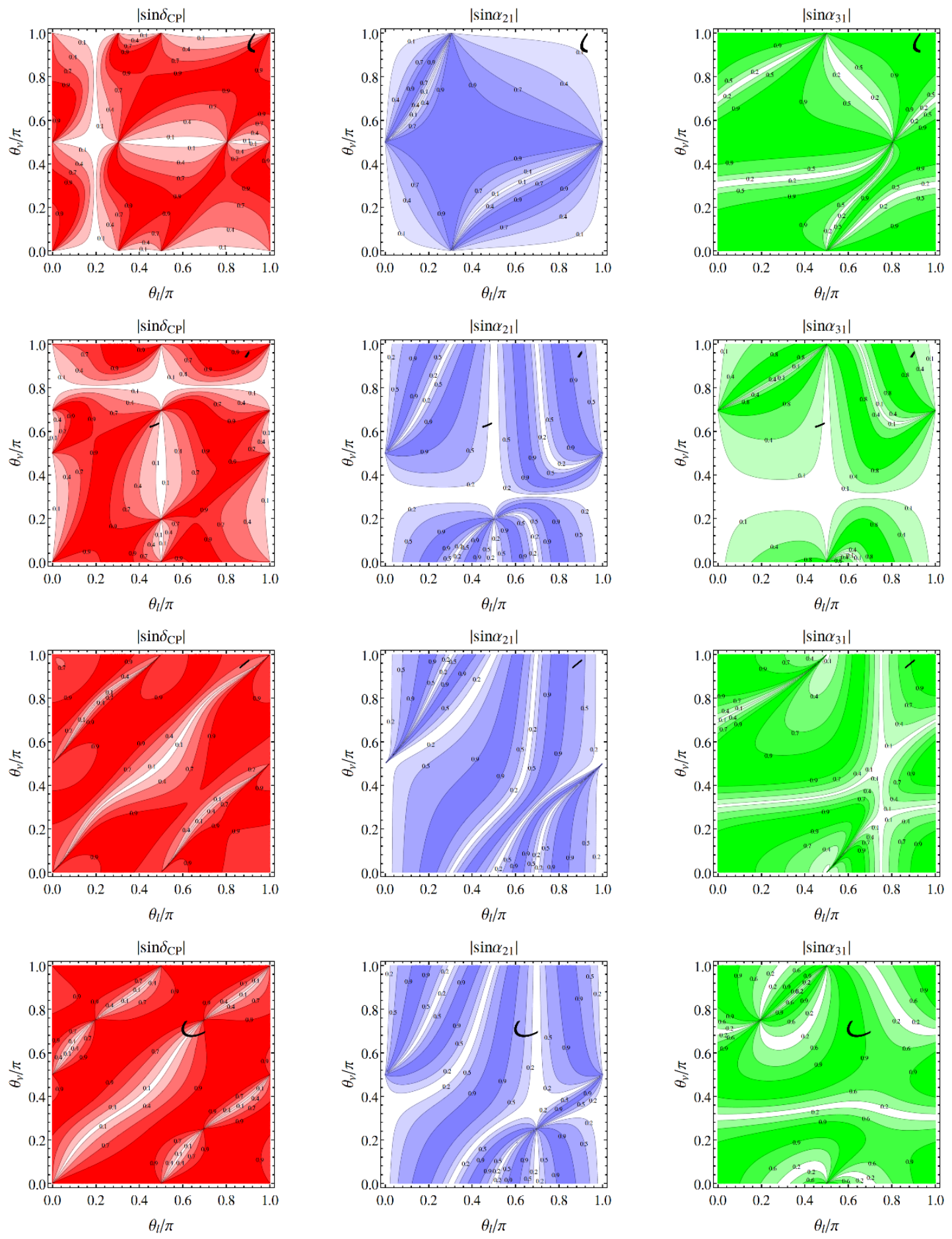

Figure 7. The contour plots of the $\mathrm{CP}$ violation phases $\left|\sin \delta_{\mathrm{CP}}\right|,\left|\sin \alpha_{21}\right|$ and $\left|\sin \alpha_{31}\right|$ for the mixing pattern $U_{\mathrm{II}, 2}$, where the parameters $\left(\varphi_{1}, \varphi_{2}\right)$ are equal to $(0, \pi / 4),(\pi / 4,0),(\pi / 4, \pi / 4)$ and $(\pi / 2, \pi / 4)$ in the four rows panels, respectively. The black areas represent the regions for which all the three lepton mixing angles lie in their corresponding experimentally allowed $3 \sigma$ intervals [8]. Since $U_{\mathrm{II}, 2}$ and $U_{\mathrm{II}, 4}$ are related through the exchange of the second and the third rows of the mixing matrix, they lead to the same Majorana phases $\alpha_{21}$ and $\alpha_{31}$ while the Dirac phase changes from $\delta_{\mathrm{CP}}$ to $\pi+\delta_{\mathrm{CP}}$. 

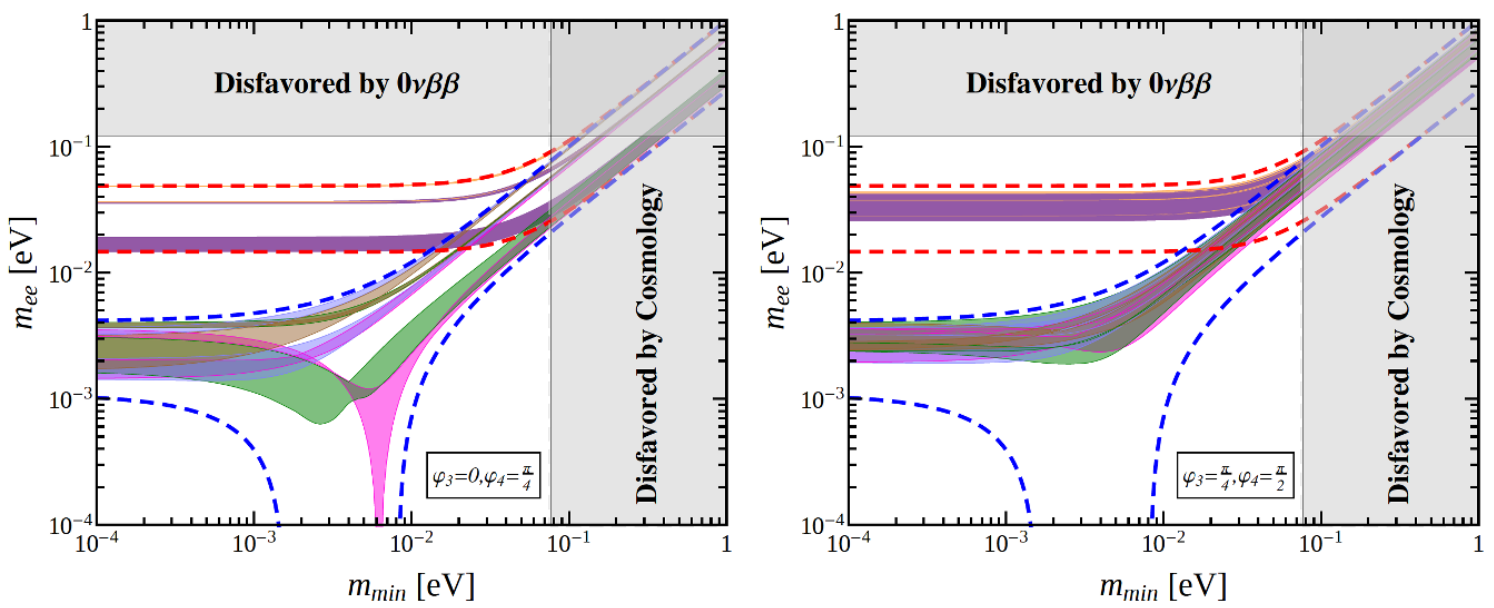

$$
\begin{aligned}
& \mathrm{IH}:\left(\mathrm{k}_{1}, \mathrm{k}_{2}\right)=(1,1),(1,-1) \quad \mathrm{IH}:\left(\mathrm{k}_{1}, \mathrm{k}_{2}\right)=(-1,1),(-1,-1) \quad \mathrm{NH}:\left(\mathrm{k}_{1}, \mathrm{k}_{2}\right)=(1,1) \\
& \mathrm{NH}:\left(\mathrm{k}_{1}, \mathrm{k}_{2}\right)=(1,-1) \square \mathrm{NH}:\left(\mathrm{k}_{1}, \mathrm{k}_{2}\right)=(-1,1) \quad \mathrm{NH}:\left(\mathrm{k}_{1}, \mathrm{k}_{2}\right)=(-1,-1)
\end{aligned}
$$

Figure 8. The possible values of the effective Majorana mass $m_{e e}$ as a function of the lightest neutrino mass $m_{\min }$ for mixing matrix $U_{\mathrm{II}, 1}$ with $n=4$. The red (blue) dashed lines indicate the most general allowed regions for IH $(\mathrm{NH})$ neutrino mass spectrum obtained by varying the mixing parameters over their $3 \sigma$ ranges [8]. The present most stringent upper limits $m_{e e}<0.120 \mathrm{eV}$ from EXO-200 [85, 86] and KamLAND-ZEN [87] is shown by horizontal grey band. The vertical grey exclusion band denotes the current bound coming from the cosmological data of $\sum m_{i}<0.230 \mathrm{eV}$ at $95 \%$ confidence level obtained by the Planck collaboration [88].

It is easy to check that $U_{\mathrm{III}}$ has the following symmetry properties:

$$
\begin{aligned}
U_{\mathrm{III}}\left(\varphi_{5}+\pi, \varphi_{6}, \theta_{l}, \theta_{\nu}\right) & =U_{\mathrm{III}}\left(\varphi_{5}, \varphi_{6}, \theta_{l}, \pi-\theta_{\nu}\right) \operatorname{diag}(-1,1,1), \\
U_{\mathrm{III}}\left(\varphi_{5}, \varphi_{6}+\pi, \theta_{l}, \theta_{\nu}\right) & =U_{\mathrm{III}}\left(\varphi_{5}, \varphi_{6}, \theta_{l}, \theta_{\nu}\right) \operatorname{diag}(1,1,-1), \\
U_{\mathrm{III}}\left(\varphi_{5}, \varphi_{6}+\frac{\pi}{2}, \theta_{l}, \theta_{\nu}\right) & =U_{\mathrm{III}}\left(\varphi_{5}, \varphi_{6}, \theta_{l}, \theta_{\nu}\right) \operatorname{diag}(1,1, i), \\
U_{\mathrm{III}}\left(\pi-\varphi_{5}, \pi-\varphi_{6}, \theta_{l}, \theta_{\nu}\right) & =U_{\mathrm{III}}^{*}\left(\varphi_{5}, \varphi_{6}, \theta_{l}, \pi-\theta_{\nu}\right) \operatorname{diag}(-1,1,-1) .
\end{aligned}
$$

Hence it is sufficient to focus on the fundamental interval of $0 \leq \varphi_{5}<\pi$ and $0 \leq \varphi_{6}<\pi / 2$. Eq. (3.40d) implies that the mixing matrix $U_{\text {III }}$ for $\pi / 2<\varphi_{5}<\pi$ is related to that of $0<\varphi_{5}<\pi / 2$ through complex conjugation. In this case, we see the magnitude of the fixed element is $1 / \sqrt{2}$ which can only be the (22), (23), (32) or (33) entries in order to achieve agreement with experimental data. As a consequence, we have four phenomenologically viable mixing patterns after the permutations of row and columns of the mixing matrix is considered,

$$
\begin{array}{ll}
U_{\mathrm{III}, 1}=P_{12} U_{\mathrm{III}} P_{23}, & U_{\mathrm{III}, 2}=P_{12} U_{\mathrm{III}}, \\
U_{\mathrm{III}, 3}=P_{23} P_{12} U_{\mathrm{III}} P_{23}, & U_{\mathrm{III}, 4}=P_{23} P_{12} U_{\mathrm{III}} .
\end{array}
$$

We notice that the PMNS matrices $U_{\mathrm{III}, 3}$ and $U_{\mathrm{III}, 4}$ can be obtained by exchanging the second and third rows of $U_{\mathrm{III}, 1}$ and $U_{\mathrm{III}, 2}$ respectively. Hence we only show the predictions of the mixing matrices $U_{\mathrm{III}, 1}$ and $U_{\mathrm{III}, 2}$ in the following. For each mixing matrix in above, the predictions for the lepton mixing angles as well as CP invariants are collected in table 8 . We see that the three lepton mixing angles and the weak basis invariants $J_{\mathrm{CP}}$ and $\mathcal{I}_{2}$ depend not only on the continuous parameters $\theta_{l, \nu}$, but also on the discrete parameter $\varphi_{5}$ whose 


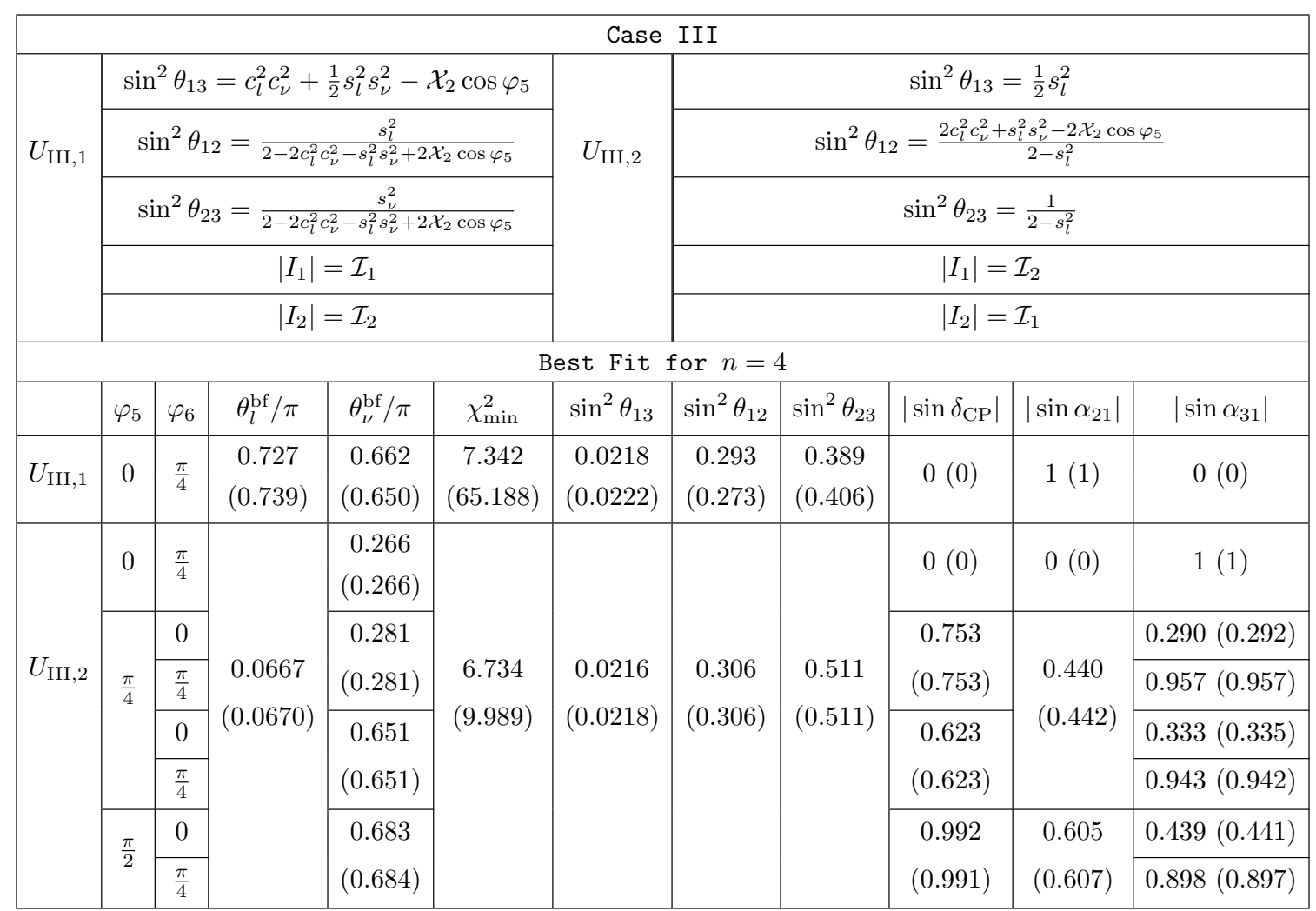

Table 8. The results for the mixing parameters in case III, and the absolute value of the Jarlskog invariant $J_{\mathrm{CP}}$ is given by $\left|J_{\mathrm{CP}}\right|=\frac{1}{8 \sqrt{2}}\left|\sin 2 \theta_{l} \sin 2 \theta_{\nu} \sin \varphi_{5}\right|$. The parameters $\mathcal{X}_{2}, \mathcal{I}_{1}$ and $\mathcal{I}_{2}$ are defined as $\mathcal{X}_{2}=\sqrt{2} c_{l} c_{\nu} s_{l} s_{\nu}, \mathcal{I}_{1}=\frac{1}{4}\left|s_{l}^{2}\left[c_{\nu}^{2} s_{l}^{2} \sin 2 \varphi_{6}-2 c_{l}^{2} s_{\nu}^{2} \sin 2\left(\varphi_{5}-\varphi_{6}\right)-2 \mathcal{X}_{2} \sin \left(\varphi_{5}-2 \varphi_{6}\right)\right]\right|$ and $\mathcal{I}_{2}=\frac{1}{2}\left|\mathcal{X}_{2} \sin \varphi_{5}\left(3 c_{l}^{2}-1+2 \sqrt{2} s_{l} c_{l} \cot 2 \theta_{\nu} \cos \varphi_{5}\right)\right|$. The $\chi^{2}$ function obtains a global minimum $\chi_{\min }^{2}$ at the best fit values $\left(\theta_{l}, \theta_{\nu}\right)=\left(\theta_{l}^{\mathrm{bf}}, \theta_{\nu}^{\mathrm{bf}}\right)$. We display the values of the mixing angles and CP violation phases at the best fitting point. Here we omitted the results with all three $\mathrm{CP}$ phases being trivial. The same values of mixing parameters as well as $\chi_{\min }^{2}$ are achieved at $\left(\theta_{l}, \theta_{\nu}\right)=$ $\left(\pi-\theta_{l}^{\mathrm{bf}}, \pi-\theta_{\nu}^{\mathrm{bf}}\right)$, because the formulae of the mixing angles and CP invariants are not changed under the transformation $\left(\theta_{l}, \theta_{\nu}\right) \rightarrow\left(\pi-\theta_{l}, \pi-\theta_{\nu}\right)$. The numbers given in parentheses are the corresponding results for the IH neutrino mass spectrum. We don't display the results for the cases of $\varphi_{5}=\varphi_{6}=0$ since all three CP phases would be trivial which is disfavored by the evidence of $\delta_{\mathrm{CP}}=3 \pi / 2[3-6]$.

value is determined by the residual symmetry. The Majorana invariant $\mathcal{I}_{1}$, which is equal to $\left|I_{1}\right|$ for $U_{\mathrm{III}, 1}$ and $\left|I_{2}\right|$ for $U_{\mathrm{III}, 2}$, is dependent not only on these three parameters, but also on a fourth discrete parameter $\varphi_{6}$. All mixing parameters are strongly correlated such that the following sum rules among the mixing angles and Dirac CP phase are found to be satisfied,

$$
\begin{array}{ll}
U_{\mathrm{III}, 2}: & \cos ^{2} \theta_{13} \sin ^{2} \theta_{23}=\frac{1}{2}, \quad U_{\mathrm{III}, 4}: \quad \cos ^{2} \theta_{13} \cos ^{2} \theta_{23}=\frac{1}{2}, \\
U_{\mathrm{III}, 1}: & \cos \delta_{\mathrm{CP}}=-\frac{1-2 \cos ^{2} \theta_{12} \cos ^{2} \theta_{23}-2 \sin ^{2} \theta_{13} \sin ^{2} \theta_{12} \sin ^{2} \theta_{23}}{\sin 2 \theta_{12} \sin \theta_{13} \sin 2 \theta_{23}}, \\
U_{\mathrm{III}, 3}: & \cos \delta_{\mathrm{CP}}=\frac{1-2 \cos ^{2} \theta_{12} \sin ^{2} \theta_{23}-2 \sin ^{2} \theta_{13} \sin ^{2} \theta_{12} \cos ^{2} \theta_{23}}{\sin 2 \theta_{12} \sin \theta_{13} \sin 2 \theta_{23}} .
\end{array}
$$



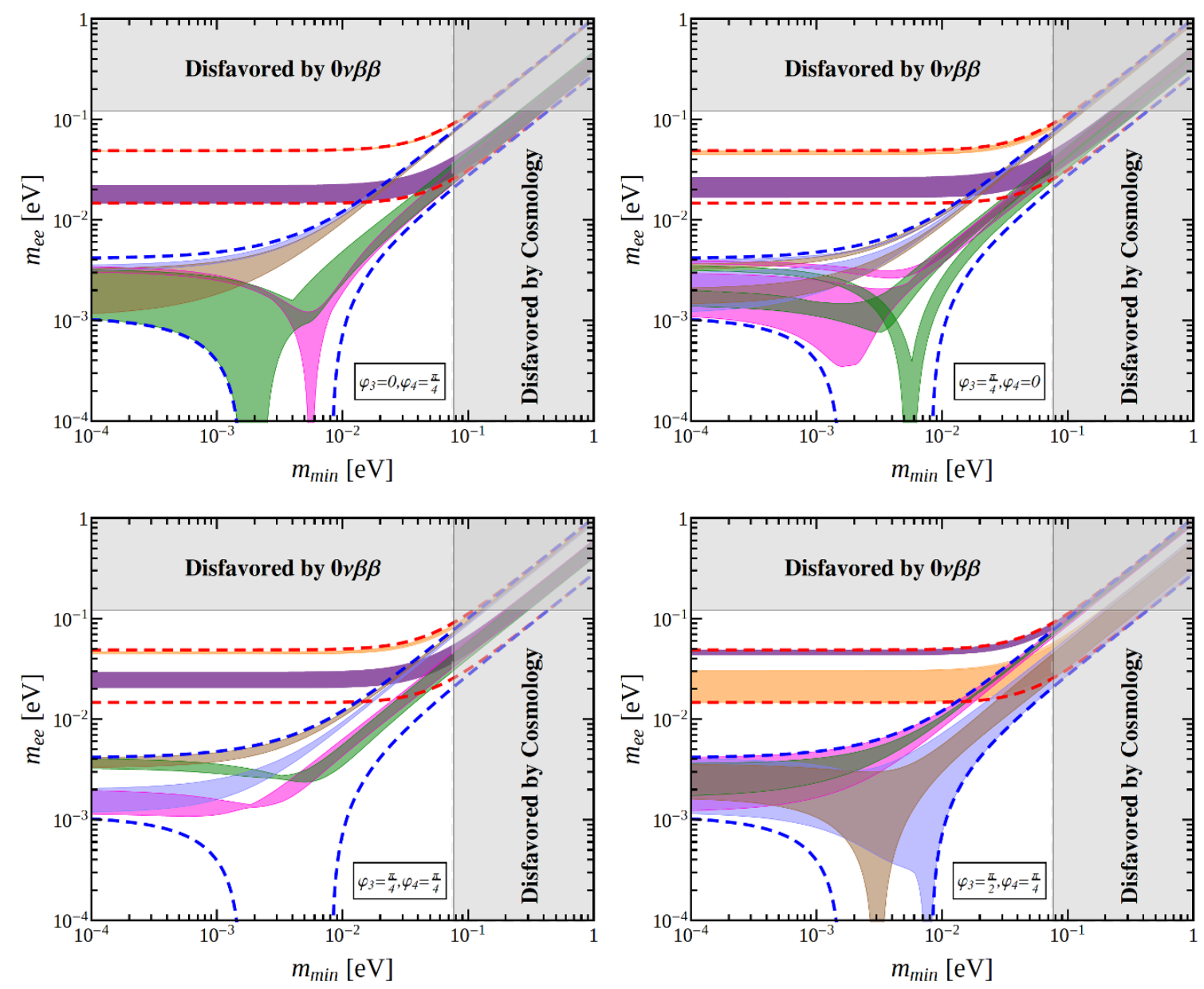

$\mathrm{IH}:\left(\mathrm{k}_{1}, \mathrm{k}_{2}\right)=(1,1),(1,-1) \quad \mathrm{IH}:\left(\mathrm{k}_{1}, \mathrm{k}_{2}\right)=(-1,1),(-1,-1)$
$\mathrm{NH}:\left(\mathrm{k}_{1}, \mathrm{k}_{2}\right)=(1,-1) \quad \mathrm{NH}:\left(\mathrm{k}_{1}, \mathrm{k}_{2}\right)=(1,1)$
$\mathrm{NH}:\left(\mathrm{k}_{1}, \mathrm{k}_{2}\right)=(-1,1) \quad \mathrm{NH}:\left(\mathrm{k}_{1}, \mathrm{k}_{2}\right)=(-1,-1)$

Figure 9. The possible values of the effective Majorana mass $m_{e e}$ as a function of the lightest neutrino mass $m_{\min }$ for mixing matrix $U_{\mathrm{II}, 2}$ with $n=4$. The red (blue) dashed lines indicate the most general allowed regions for IH $(\mathrm{NH})$ neutrino mass spectrum obtained by varying the mixing parameters over their $3 \sigma$ ranges [8]. The present most stringent upper limits $m_{e e}<0.120 \mathrm{eV}$ from EXO-200 [85, 86] and KamLAND-ZEN [87] is shown by horizontal grey band. The vertical grey exclusion band denotes the current bound coming from the cosmological data of $\sum m_{i}<0.230 \mathrm{eV}$ at $95 \%$ confidence level obtained by the Planck collaboration [88].

These sum rules for $\cos \delta_{\mathrm{CP}}$ are also derived in ref. [63]. But the predictions for the three mixing angles are different, consequently the allowed regions of $\cos \delta_{\mathrm{CP}}$ in this case don't coincide with those of ref. [63]. If the three mixing angles vary in the $3 \sigma$ ranges, the allowed regions of $\cos \delta_{\mathrm{CP}}$ are found to be $[-1,-0.735]$ and $[0.525,1]$ for $U_{\mathrm{III}, 1}$ and $U_{\mathrm{III}, 3}$ respectively. As a result, both mixing patterns would be excluded if the Dirac CP phase $\delta_{\mathrm{CP}}$ is measured to be around maximal value. Given the measured reactor mixing angle $\sin ^{2} \theta_{13} \simeq 0.02166$ [8], we find for the atmospheric mixing angle

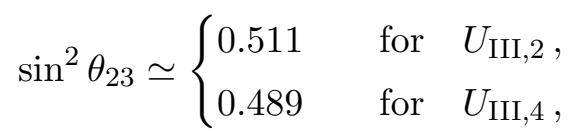


which deviates slightly from maximal mixing. It is remarkable that a good fit to the experimental data can always be achieved for any $\Delta\left(6 n^{2}\right)$ flavor group with even $n$ in this case. The $\Delta\left(6 \cdot 2^{2}\right) \cong S_{4}$ group has been comprehensively analyzed in [60], two independent sets of values $\left(\varphi_{5}, \varphi_{6}\right)=(0,0),(\pi / 2,0)$ are admissible, and they correspond to $\left(X_{l}, X_{\nu}\right)=\left(T^{2}, T S T^{2} U\right)$ and $\left(T^{2}, S U\right)$ respectively with $\left(G_{l}, G_{\nu}\right)=\left(Z_{2}^{S T^{2} S U}, Z_{2}^{S}\right)$ in the notation of [60]. In order to show new interesting mixing patterns, here we shall consider the next flavor group with $n=4$. The possible values of $\varphi_{5,6}$ are $\left(\varphi_{5}, \varphi_{6}\right)=(0,0)$, $(0, \pi / 4),(\pi / 4,0),(\pi / 4, \pi / 4),(\pi / 2,0)$ and $(\pi / 2, \pi / 4) .^{2}$ From the explicit form of the mixing matrix shown in eq. (3.39), we see that for the same value of $\varphi_{5}, \varphi_{6}=0$ and $\varphi_{6}=\pi / 4$ lead to the same mixing angles and Dirac CP phase, while the Majorana phase $\alpha_{21}\left(\alpha_{31}\right)$ differs by $\pi / 2$ for the mixing patterns $U_{\mathrm{III}, 1}\left(U_{\mathrm{III}, 2}\right)$. In other words the predictions of $\varphi_{6}=\pi / 4$ can be read from those of $\varphi_{6}=0$. As a result, it is sufficient to consider the cases with $\varphi_{6}=0$. For $\varphi_{5}=\varphi_{6}=0$, all the four permutations in eq. (3.41) can describe the experimentally measured values of the mixing angles for certain choices of $\theta_{l}$ and $\theta_{\nu}$. Both Dirac and Majorana CP phases are trivial since the mixing matrix is real. From the view of symmetry, $\varphi_{5}=\varphi_{6}=0$ requires $\alpha=\gamma$ and $\delta=-2 x-\gamma$. As a consequence, $X=c^{\gamma} d^{-2 x-\gamma}$ is an accidental CP symmetry of the charged lepton and neutrino mass matrices and therefore all three CP phases are trivial. For the values of $\varphi_{5}=\pi / 4, \varphi_{6}=0$ and $\varphi_{5}=\pi / 2, \varphi_{6}=0$, only the mixing patterns $U_{\mathrm{III}, 2}$ can accommodate the experimental data on mixing angles, they are equivalent to the mixing matrices $U_{\mathrm{I}, 6}$ with $\left(\varphi_{1}, \varphi_{2}\right)=(\pi / 4, \pi / 4)$ and $\left(\varphi_{1}, \varphi_{2}\right)=(\pi / 4, \pi / 2)$ respectively. Hence the contour regions of $\sin ^{2} \theta_{i j}(i j=12,13,23)$ and the $\mathrm{CP}$ violating phases $\left|\sin \delta_{\mathrm{CP}}\right|,\left|\sin \alpha_{21}\right|,\left|\sin \alpha_{31}\right|$ in the plane $\theta_{\nu}$ versus $\theta_{l}$ for these two cases are similar to figure 1 and figure 2 , respectively. We shall not show them here. Furthermore, we report the best fit values of the mixing parameters and the global minimum of $\chi^{2}$ for each case in table 8. Requiring the three mixing angles are in the experimentally preferred $3 \sigma$ ranges, the allowed regions of $\left|\sin \delta_{\mathrm{CP}}\right|$, $\left|\sin \alpha_{21}\right|$ and $\left|\sin \alpha_{31}\right|$ are exactly given by eq. (3.19) and eq. (3.20) for $\left(\varphi_{5}, \varphi_{6}\right)=(\pi / 4,0)$ and $\left(\varphi_{5}, \varphi_{6}\right)=(\pi / 2,0)$ respectively. From the numerical results in table 8 , we see that the two mixing patterns $U_{\mathrm{III}, 1}$ and $U_{\mathrm{III}, 2}$ should be distinguishable from each other at future neutrino facilities, since their predictions for the mixing angles $\theta_{12}$ and $\theta_{23}$ and the Dirac phase $\delta_{\mathrm{CP}}$ are quite different. For the mixing pattern $U_{\mathrm{III}, 2}$, different values of $\varphi_{5}$ (e.g. $\left.\varphi_{5}=0, \pi / 4, \pi / 2\right)$ lead to the same lepton mixing angles while the prediction for Dirac and Majorana CP phases are distinct. A discrimination between different cases are possible if the Dirac CP phase is measured precisely enough at forthcoming long baseline experiments or neutrino factory. For a given $\varphi_{5}$, the mixing matrices $U_{\mathrm{III}, 1}\left(U_{\mathrm{III}, 2}\right)$ corresponding to different values of $\varphi_{6}$ only differ in the Majorana phase $\alpha_{31}\left(\alpha_{21}\right)$, the predictions for the other mixing parameters are identical. Thus it is unlikely that they can be distinguished at future neutrino facilities except that Majorana CP phases could be marvelously measured. As regards the neutrinoless double decay, the effective mass $m_{e e}$ takes the form

$$
m_{e e}=\frac{1}{2}\left|\left(s_{l} c_{\nu}+\sqrt{2} e^{i \varphi_{5}} c_{l} s_{\nu}\right)^{2} m_{1}+k_{1} e^{2 i \varphi_{6}} s_{l}^{2} m_{2}+k_{2}\left(s_{l} s_{\nu}-\sqrt{2} e^{i \varphi_{5}} c_{l} c_{\nu}\right)^{2} m_{3}\right|
$$

\footnotetext{
${ }^{2}$ The values $\left(\varphi_{5}, \varphi_{6}\right)=(3 \pi / 4,0),(3 \pi / 4, \pi / 4)$ are admissible as well, and the resulting mixing matrices are the complex conjugates of the ones for $\left(\varphi_{5}, \varphi_{6}\right)=(\pi / 4,0),(\pi / 4, \pi / 4)$ respectively up to redefinition of $\theta_{\nu}$ and $Q_{\nu}$. Therefore the same mixing angles are obtained and the overall signs of the CP phases are reversed.
} 

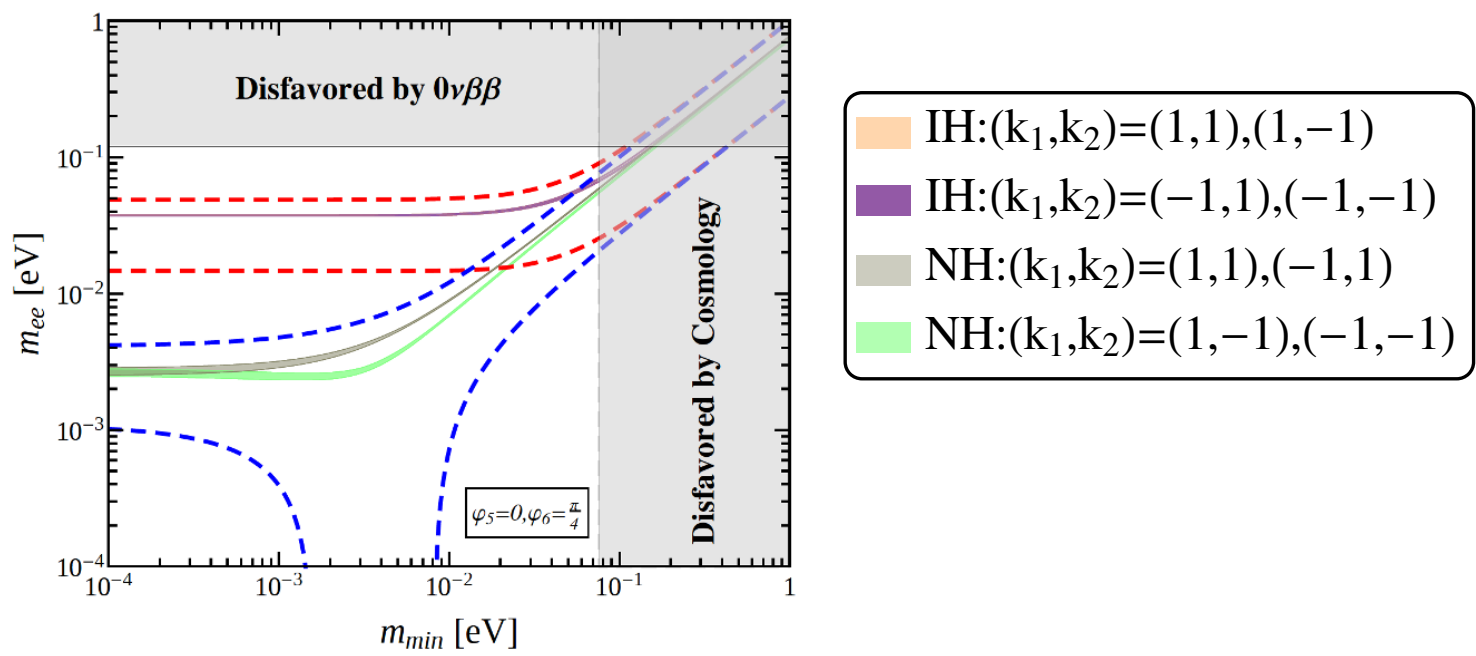

Figure 10. The possible values of the effective Majorana mass $m_{e e}$ as a function of the lightest neutrino mass $m_{\min }$ for the mixing patterns $U_{\mathrm{III}, 1}$ and $U_{\mathrm{III}, 3}$. The red (blue) dashed lines indicate the most general allowed regions for IH $(\mathrm{NH})$ neutrino mass spectrum obtained by varying the mixing parameters over their $3 \sigma$ ranges [8]. The present most stringent upper limits $m_{e e}<0.120 \mathrm{eV}$ from EXO-200 [85, 86] and KamLAND-ZEN [87] is shown by horizontal grey band. The vertical grey exclusion band denotes the current bound coming from the cosmological data of $\sum m_{i}<0.230 \mathrm{eV}$ at $95 \%$ confidence level obtained by the Planck collaboration [88].

for $U_{\mathrm{III}, 1}$ and

$$
m_{e e}=\frac{1}{2}\left|\left(s_{l} c_{\nu}+\sqrt{2} e^{i \varphi_{5}} c_{l} s_{\nu}\right)^{2} m_{1}+k_{1}\left(s_{l} s_{\nu}-\sqrt{2} e^{i \varphi_{5}} c_{l} c_{\nu}\right)^{2} m_{2}+k_{2} e^{2 i \varphi_{6}} s_{l}^{2} m_{3}\right|,
$$

for $U_{\mathrm{III}, 2}$. We show the corresponding predictions for the $(\beta \beta)_{0 \nu}$-decay effective mass as a function of the lightest neutrino mass in figure 10 and figure 11. For the mixing matrix $U_{\text {III, } 1}$ with $\left(\varphi_{5}, \varphi_{6}\right)=(0, \pi / 4)$, we see that the effective Majorana mass $m_{e e}$ is around $0.037 \mathrm{eV}$ and $0.0026 \mathrm{eV}$ for hierarchical $\mathrm{IH}$ and $\mathrm{NH}$ spectrums, respectively. For the mixing pattern $U_{\mathrm{III}, 2}$, strong cancellations doesn't occur and the effective mass $m_{e e}$ has a lower limit given by $m_{e e}>10^{-3} \mathrm{eV}$ for the parameter values $\left(\varphi_{5}, \varphi_{6}\right)=(0, \pi / 4),(\pi / 4, \pi / 4)$, $(\pi / 2,0)$. Notice that the shaded areas in figure 10 are much smaller than in the other figures. This is because the three mixing angles are found to lie in rather narrow regions,

$$
0.01934 \leq \sin ^{2} \theta_{13} \leq 0.02392, \quad 0.271 \leq \sin ^{2} \theta_{12} \leq 0.304, \quad 0.385 \leq \sin ^{2} \theta_{23} \leq 0.406,
$$

for the mixing pattern $U_{\mathrm{III}, 1}$ with $\left(\varphi_{5}, \varphi_{6}\right)=(0, \pi / 4)$, the $\mathrm{CP}$ violation phases $\delta_{\mathrm{CP}}$ and $\alpha_{31}$ are conserved and $\alpha_{21}$ is maximal, as shown in table 8 .

(IV) $Z_{2}^{g_{l}}=Z_{2}^{c^{n / 2}}, X_{l}=\left\{c^{\alpha} d^{\delta}\right\}, Z_{2}^{g_{\nu}}=Z_{2}^{b c^{x} d^{x}}, X_{\nu}=\left\{c^{\gamma} d^{-2 x-\gamma}, b c^{x+\gamma} d^{-x-\gamma}\right\}$. Compared with Case III, the residual symmetries in the neutrino and charged lepton sectors are interchanged, consequently the $\Sigma$ matrix is the hermitian conjugate of the one in 

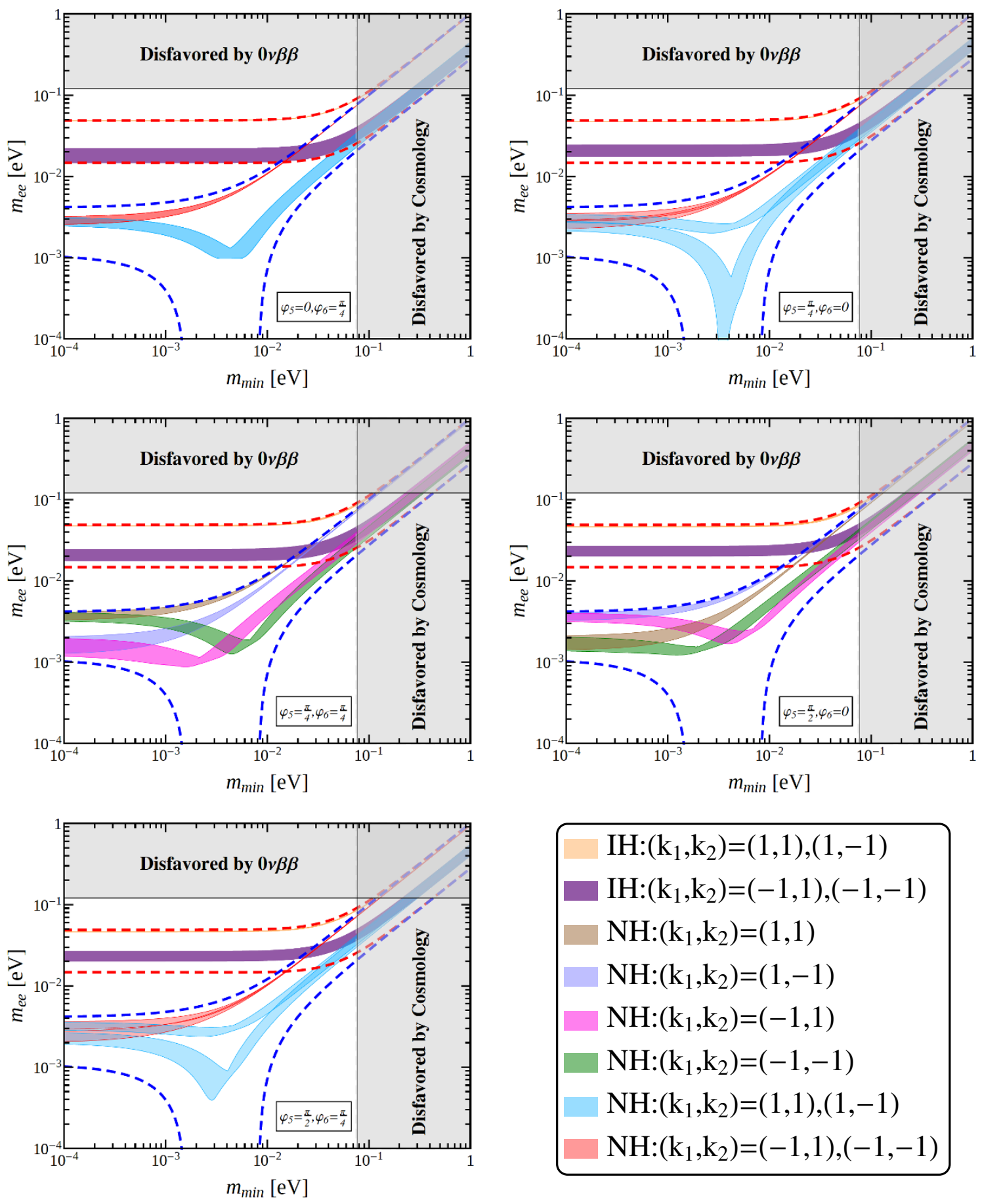

$\mathrm{IH}:\left(\mathrm{k}_{1}, \mathrm{k}_{2}\right)=(1,1),(1,-1)$
$\mathrm{IH}:\left(\mathrm{k}_{1}, \mathrm{k}_{2}\right)=(-1,1),(-1,-1)$
$\mathrm{NH}:\left(\mathrm{k}_{1}, \mathrm{k}_{2}\right)=(1,1)$
$\mathrm{NH}:\left(\mathrm{k}_{1}, \mathrm{k}_{2}\right)=(1,-1)$
$\mathrm{NH}:\left(\mathrm{k}_{1}, \mathrm{k}_{2}\right)=(-1,1)$
$\mathrm{NH}:\left(\mathrm{k}_{1}, \mathrm{k}_{2}\right)=(-1,-1)$
$\mathrm{NH}:\left(\mathrm{k}_{1}, \mathrm{k}_{2}\right)=(1,1),(1,-1)$
$\mathrm{NH}:\left(\mathrm{k}_{1}, \mathrm{k}_{2}\right)=(-1,1),(-1,-1)$

Figure 11. The possible values of the effective Majorana mass $m_{e e}$ as a function of the lightest neutrino mass $m_{\min }$ for the mixing patterns $U_{\mathrm{III}, 2}$ and $U_{\mathrm{III}, 4}$. The red (blue) dashed lines indicate the most general allowed regions for IH $(\mathrm{NH})$ neutrino mass spectrum obtained by varying the mixing parameters over their $3 \sigma$ ranges [8]. The present most stringent upper limits $m_{e e}<0.120 \mathrm{eV}$ from EXO-200 [85, 86] and KamLAND-ZEN [87] is shown by horizontal grey band. The vertical grey exclusion band denotes the current bound coming from the cosmological data of $\sum m_{i}<0.230 \mathrm{eV}$ at $95 \%$ confidence level obtained by the Planck collaboration [88]. 
eq. (3.36), i.e.

$$
\Sigma=\frac{1}{\sqrt{2}}\left(\begin{array}{ccc}
e^{-i \varphi_{6}} & e^{-i \varphi_{6}} & 0 \\
0 & 0 & \sqrt{2} e^{-i \varphi_{5}} \\
-1 & 1 & 0
\end{array}\right)
$$

where the discrete parameters $\varphi_{5}$ and $\varphi_{6}$ are given in eq. (3.37). Subsequently we can read out PMNS mixing matrix as

$$
U_{\mathrm{IV}}=\frac{1}{\sqrt{2}}\left(\begin{array}{ccc}
c_{l} & c_{l} s_{\nu}+\sqrt{2} e^{-i \varphi_{5}} s_{l} c_{\nu} & c_{l} c_{\nu}-\sqrt{2} e^{-i \varphi_{5}} s_{l} s_{\nu} \\
s_{l} & s_{l} s_{\nu}-\sqrt{2} e^{-i \varphi_{5}} c_{l} c_{\nu} & s_{l} c_{\nu}+\sqrt{2} e^{-i \varphi_{5}} c_{l} s_{\nu} \\
-1 & s_{\nu} & c_{\nu}
\end{array}\right),
$$

up to row and column permutations. Note that the phase $e^{i \varphi_{6}}$ has been absorbed into the charged lepton fields. Moreover, we find that the mixing matrices $U_{\mathrm{IV}}$ and $U_{\mathrm{III}}$ are closely related with each other as follows

$$
U_{\mathrm{IV}}\left(\varphi_{5}, \theta_{l}, \theta_{\nu}\right)=P_{13} U_{\mathrm{III}}\left(\pi-\varphi_{5}, \varphi_{6}=0, \theta_{l}, \pi-\theta_{\nu}\right) P_{13} \operatorname{diag}(1,1,-1) .
$$

Since we have considered all possible values of $\varphi_{5,6}$ and all possible permutations of rows and columns in case III, therefore we don't obtain additional new results in the present case.

In short, the possible mixing patterns which can be obtained from $\Delta\left(6 n^{2}\right)$ group in our approach vary with the group index $n$. If $n^{\prime}$ is divisible by $n, \Delta\left(6 n^{2}\right)$ would be a subgroup of $\Delta\left(6 n^{\prime 2}\right)$, and consequently all the mixing patterns arising from the $\Delta\left(6 n^{2}\right)$ group can also be obtained from $\Delta\left(6 n^{\prime 2}\right)$. The future neutrino oscillation experiments such as JUNO [95], RENO-50 [96], DUNE [76-79], T2HK [80, 81], T2HKK [82] etc can make very precise measurements of the oscillation parameters $\theta_{12}, \theta_{23}$ and $\delta_{\mathrm{CP}}$. The next generation of $(\beta \beta)_{0 \nu}$-decay experiments could probe the full region of parameter space associated with $\mathrm{IH}$ neutrino mass spectrum. Thus we expect that future experimental programme can provide evidence for the relevance of certain mixing patterns, and eventually help us to find out which flavor symmetry describes data best. For the sake of simplicity, we are interested in the smallest $\Delta\left(6 n^{2}\right)$ group which can accommodate the experimental data.

\section{Quark mixing from $\Delta\left(6 n^{2}\right)$ and CP symmetries}

So far each element of the CKM mixing matrix $V$ has been measured to a good degree of accuracy [1], the global fit results for the moduli of all the nine CKM elements are [1],

$$
|V|=\left(\begin{array}{ccc}
0.97434_{-0.00012}^{+0.00011} & 0.22506 \pm 0.00050 & 0.00357 \pm 0.00015 \\
0.22492 \pm 0.00050 & 0.97351 \pm 0.00013 & 0.0411 \pm 0.0013 \\
0.00875_{-0.00033}^{+0.00032} & 0.0403 \pm 0.0013 & 0.99915 \pm 0.00005
\end{array}\right)
$$

In contrast with the more or less "anarchical" structure of the lepton mixing matrix, the quark CKM mixing matrix has a clear hierarchy structure $\left|V_{t b}\right|>\left|V_{u d}\right|>\left|V_{c s}\right| \gg\left|V_{u s}\right|>$ 
$\left|V_{c d}\right| \gg\left|V_{c b}\right|>\left|V_{t s}\right| \gg\left|V_{t d}\right|>\left|V_{u b}\right|$. Combining all available measurements of CP violation and rare decays in the quark sector, the UTfit collaboration gives $[100,101]^{3}$

$$
\begin{aligned}
\sin \theta_{12}^{q} & =0.22497 \pm 0.00069, \quad \sin \theta_{23}^{q}=0.04229 \pm 0.00057, \\
\sin \theta_{13}^{q} & =0.00368 \pm 0.00010, \quad J_{\mathrm{CP}}^{q}=(3.115 \pm 0.093) \times 10^{-5},
\end{aligned}
$$

where the superscript " $q$ " means that these quantities describe the quark mixing and $\mathrm{CP}$ violation. In this section, we shall investigate whether it is also possible to derive phenomenologically viable quark mixing from $\Delta\left(6 n^{2}\right)$ flavor group and CP symmetry in the same way, as presented for the lepton sector in section 3 .

The original $\Delta\left(6 n^{2}\right)$ and CP symmetries are assumed to be broken down to the residual subgroups $Z_{2}^{g_{u}} \times X_{u}$ and $Z_{2}^{g_{d}} \times X_{d}$ in the up quark and down quark sectors respectively, then the CKM mixing matrix would be constrained to take the form of eq. (2.23). Note that the CKM mixing matrix depends on two free parameters $\theta_{u}$ and $\theta_{d}$ and one element independent of $\theta_{u, d}$ is fixed in this framework. In the same fashion as the lepton sector, for all the residual subgroups of the structure $Z_{2} \times C P$, the corresponding Takagi factorization matrices $\Sigma_{u}$ or $\Sigma_{d}$ are summarized in table 1. Furthermore, considering all possible residual symmetries $Z_{2}^{g_{u}} \times X_{u}$ and $Z_{2}^{g_{d}} \times X_{d}$, the fixed element is determined to be $0,1,1 / 2,1 / \sqrt{2}$ or $\cos \varphi_{1}$. Taking into account the current data in eq. (4.1), only the mixing pattern with the fixed element $\cos \varphi_{1}$ could be in agreement with experimental data for certain values of $\varphi_{1}$ characterizing the residual symmetry. ${ }^{4}$ As a consequence, the unique viable residual symmetries in the quark sector are $Z_{2}^{g_{u}}=Z_{2}^{b c^{x} d^{x}}, X_{u}=\left\{c^{\gamma} d^{-2 x-\gamma}, b c^{x+\gamma} d^{-x-\gamma}\right\}, Z_{2}^{g_{d}}=$ $Z_{2}^{b c^{y} d^{y}}$ and $X_{d}=\left\{c^{\delta} d^{-2 y-\delta}, b c^{y+\delta} d^{-y-\delta}\right\}$ where $x, y, \gamma, \delta=0,1, \ldots, n-1$. Accordingly the CKM mixing matrix reads

$$
V_{\mathrm{I}}=\left(\begin{array}{ccc}
\cos \varphi_{1} & s_{d} \sin \varphi_{1} & -c_{d} \sin \varphi_{1} \\
-s_{u} \sin \varphi_{1} & c_{u} c_{d} e^{i \varphi_{2}}+s_{u} s_{d} \cos \varphi_{1} & c_{u} s_{d} e^{i \varphi_{2}}-c_{d} s_{u} \cos \varphi_{1} \\
c_{u} \sin \varphi_{1} & c_{d} s_{u} e^{i \varphi_{2}}-c_{u} s_{d} \cos \varphi_{1} & s_{u} s_{d} e^{i \varphi_{2}}+c_{u} c_{d} \cos \varphi_{1}
\end{array}\right)
$$

with

$$
\varphi_{1}=\frac{x-y}{n} \pi, \quad \varphi_{2}=\frac{3(x-y+\gamma-\delta)}{n} \pi .
$$

Here we have omitted the diagonal phase matrices $Q_{u}, Q_{d}$ and the permutation matrices $P_{u}, P_{d}$, the abbreviations $s_{u}, s_{d}, c_{u}$ and $c_{d}$ denote

$$
s_{u} \equiv \sin \theta_{u}, \quad s_{d} \equiv \sin \theta_{d}, \quad c_{u} \equiv \cos \theta_{u}, \quad c_{d} \equiv \cos \theta_{d} .
$$

We see that the mixing matrix $V_{\mathrm{I}}$ coincides with $U_{\mathrm{I}}$ after performing the transformations $\theta_{l} \rightarrow \theta_{u}$ and $\theta_{\nu} \rightarrow \theta_{d}$. The symmetry relations in eq. (3.13) are also valid for the present quark mixing pattern $V_{\mathrm{I}}$. As a result, we shall focus on fundamental ranges of $0 \leq \varphi_{1} \leq \pi / 2$

\footnotetext{
${ }^{3}$ The results of the global data fitting from the UTfit collaboration, see webpage: http://www.utfit.org/ UTfit/ResultsSummer2016SM.

${ }^{4}$ It is a good lead order approximation that the fixed element is 0 in the (13) or (31) entry of the CKM matrix [60]. However, the quark CP violation phase would be undeterminable. We shall not discuss this case in the present work since our main motivation is to investigate whether the lepton mixing angles together with the precisely measured CKM matrix can be explained from a single flavor symmetry.
} 
and $0 \leq \varphi_{2}<\pi$ in the following. Since the order of the quark masses is undefined in our framework, the CKM matrix is determined up to independent row and column permutations. It turns out that all possible permutations of rows and columns lead to nine independent mixing patterns

$$
\begin{aligned}
& V_{\mathrm{I}, 1}=V_{\mathrm{I}}, \quad V_{\mathrm{I}, 2}=V_{\mathrm{I}} P_{12}, \quad V_{\mathrm{I}, 3}=V_{\mathrm{I}} P_{13}, \\
& V_{\mathrm{I}, 4}=P_{12} V_{\mathrm{I}}, \quad V_{\mathrm{I}, 5}=P_{12} U_{\mathrm{I}} P_{12}, \quad V_{\mathrm{I}, 6}=P_{12} V_{\mathrm{I}} P_{13}, \\
& V_{\mathrm{I}, 7}=P_{13} V_{\mathrm{I}}, \quad V_{\mathrm{I}, 8}=P_{13} V_{\mathrm{I}} P_{12}, \quad V_{\mathrm{I}, 9}=P_{13} V_{\mathrm{I}} P_{13} .
\end{aligned}
$$

We can get the expressions of the quark mixing angles and Jarlskog invariant from table 3 by simply redefining $\theta_{l} \rightarrow \theta_{u}$ and $\theta_{\nu} \rightarrow \theta_{d}$. Hence the sum rules among the mixing angles and $\mathrm{CP}$ violation phase shown in eq. (3.17) are satisfied as well in the quark sector.

In the following, we shall study numerically quark mixing angles and CP invariant which can be obtained in this case. We have evaluated for $n \leq 40$ and all corresponding values of $\varphi_{1,2}$ whether the continuous parameters $\theta_{u, d}$ can take values such that a good fit to the experimental data can be achieved. The results of this analysis are summarized in table 9 and table 10, where we list the values of $n$ and the results for the quark mixing angles $\sin \theta_{i j}^{q}$ as well as the values of the Jarlskog invariant $J_{\mathrm{CP}}^{q}$ at certain representative values of $\theta_{u, d}$. We find that only the mixing matrices $V_{\mathrm{I}, 1}, V_{\mathrm{I}, 2}, V_{\mathrm{I}, 6}$ and $V_{\mathrm{I}, 8}$ can describe the experimentally measured values of quark flavor mixing from the $\Delta\left(6 n^{2}\right)$ group with $n \leq 40$. For a good agreement with the experimental data the index $n$ has to be at least $n=7$, the corresponding CKM mixing matrix is of the form $V_{\mathrm{I}, 2}$ whose (12) entry is $\cos \varphi_{1}$. Accordingly we find the expressions of the mixing angles and $\mathrm{CP}$ invariant are

$$
\begin{aligned}
\sin ^{2} \theta_{13}^{q} & =\sin ^{2} \varphi_{1} \cos ^{2} \theta_{d}, \quad \sin ^{2} \theta_{12}^{q}=\frac{\cos ^{2} \varphi_{1}}{1-\sin ^{2} \varphi_{1} \cos ^{2} \theta_{d}} \\
\sin ^{2} \theta_{23}^{q} & =\frac{2 \cos ^{2} \theta_{u} \sin ^{2} \theta_{d}+2 \sin ^{2} \theta_{u} \cos ^{2} \theta_{d} \cos ^{2} \varphi_{1}-\cos \varphi_{1} \cos \varphi_{2} \sin 2 \theta_{u} \sin 2 \theta_{d}}{2-2 \cos ^{2} \theta_{d} \sin ^{2} \varphi_{1}} \\
J_{\mathrm{CP}}^{q} & =\frac{1}{8} \sin \varphi_{1} \sin 2 \varphi_{1} \sin \varphi_{2} \sin 2 \theta_{u} \sin 2 \theta_{d}
\end{aligned}
$$

which yield the correlations

$$
\begin{aligned}
\cos ^{2} \theta_{13}^{q} \sin ^{2} \theta_{12}^{q} & =\cos ^{2} \varphi_{1}, \\
\sin ^{2} \theta_{23}^{q} & =\frac{1}{2}-\frac{2 J_{\mathrm{CP}}^{q} \cot \varphi_{2}}{\sin ^{2} \varphi_{1} \cos ^{2} \theta_{13}^{q}} \pm \sqrt{1-4 x^{2}}\left(\frac{1}{2}-\cot ^{2} \varphi_{1} \tan ^{2} \theta_{13}^{q}\right),
\end{aligned}
$$

with

$$
x=\frac{J_{\mathrm{CP}}^{q}}{\sin \varphi_{2} \cos \varphi_{1} \sin \theta_{13}^{q} \sqrt{\sin ^{2} \varphi_{1}-\sin ^{2} \theta_{13}^{q}}} .
$$

The "+" sign in eq. (4.8b) is satisfied for $\theta_{u} \in[0, \pi / 4] \cup[3 \pi / 4, \pi]$ and "-" for $\theta_{u} \in$ $(\pi / 4,3 \pi / 4)$. The small mixing angle $\theta_{23}^{q}$ can only be obtained for the "-" sign, and thus eq. (4.8b) implies that $\sin \delta_{\mathrm{CP}}$ satisfies the following sum rule:

$$
\sin \delta_{\mathrm{CP}} \simeq \frac{\sin 2 \varphi_{1} \sin \varphi_{2}}{\sin 2 \theta_{12}^{q} \cos ^{2} \theta_{13}^{q} \cos \theta_{23}^{q}} .
$$




\begin{tabular}{|c|c|c|c|c|c|c|c|c|c|}
\hline & $n$ & $\varphi_{1}$ & $\varphi_{2}$ & $\theta_{u} / \pi$ & $\theta_{d} / \pi$ & $\sin \theta_{13}^{q}$ & $\sin \theta_{12}^{q}$ & $\sin \theta_{23}^{q}$ & $J_{\mathrm{CP}}^{q} / 10^{-5}$ \\
\hline \multirow{9}{*}{$V_{\mathrm{I}, 1}$} & \multirow{4}{*}{14,28} & \multirow{9}{*}{$\frac{\pi}{14}$} & $\frac{\pi}{2}$ & 0.48755 & 0.50526 & 0.00368 & \multirow{9}{*}{0.22249} & 0.04228 & 3.117 \\
\hline & & & $\frac{4 \pi}{7}$ & 0.48644 & 0.50512 & 0.00358 & & 0.04197 & 3.219 \\
\hline & & & $\frac{9 \pi}{14}$ & 0.48524 & 0.50510 & 0.00353 & & 0.04153 & 3.162 \\
\hline & & & $\frac{5 \pi}{7}$ & 0.48396 & 0.50524 & 0.00367 & & 0.04226 & 3.127 \\
\hline & \multirow{5}{*}{28} & & $\frac{13 \pi}{28}$ & 0.51194 & 0.49464 & 0.00374 & & 0.04258 & 3.025 \\
\hline & & & $\frac{15 \pi}{28}$ & 0.48701 & 0.50518 & 0.00362 & & 0.04208 & 3.181 \\
\hline & & & $\frac{17 \pi}{28}$ & 0.51415 & 0.49491 & 0.00357 & & 0.04195 & 3.233 \\
\hline & & & $\frac{19 \pi}{28}$ & 0.48462 & 0.50515 & 0.00360 & & 0.04210 & 3.189 \\
\hline & & & $\frac{3 \pi}{4}$ & 0.48328 & 0.50524 & 0.00367 & & 0.04226 & 3.127 \\
\hline \multirow{23}{*}{$V_{\mathrm{I}, 2}$} & $7,14,21$ & & $\frac{3 \pi}{7}$ & 0.48656 & 0.49883 & 0.00359 & \multirow{23}{*}{0.22252} & 0.04204 & 3.202 \\
\hline & 28,35 & & $\frac{4 \pi}{7}$ & 0.48666 & 0.49882 & 0.00360 & & 0.04208 & 3.190 \\
\hline & & & $\frac{5 \pi}{14}$ & 0.48640 & 0.49879 & 0.00370 & & 0.04236 & 3.090 \\
\hline & 14,28 & & $\frac{\pi}{2}$ & 0.48665 & 0.49884 & 0.00356 & & 0.04195 & 3.233 \\
\hline & & & $\frac{9 \pi}{14}$ & 0.48661 & 0.49878 & 0.00373 & & 0.04243 & 3.063 \\
\hline & & & $\frac{9 \pi}{28}$ & 0.48629 & 0.49876 & 0.00379 & & 0.04260 & 2.995 \\
\hline & & & $\frac{11 \pi}{28}$ & 0.48649 & 0.49881 & 0.00364 & & 0.04217 & 3.158 \\
\hline & 28 & & $\frac{13 \pi}{28}$ & 0.51339 & 0.50116 & 0.00356 & & 0.04197 & 3.227 \\
\hline & & $\frac{3 \pi}{4}$ & $\frac{15 \pi}{28}$ & 0.48666 & 0.49883 & 0.00357 & & 0.04199 & 3.221 \\
\hline & & & $\frac{17 \pi}{28}$ & 0.48665 & 0.49881 & 0.00366 & & 0.04223 & 3.138 \\
\hline & & & $\frac{19 \pi}{28}$ & 0.48655 & 0.49875 & 0.00382 & & 0.04270 & 2.959 \\
\hline & \multirow{12}{*}{35} & & $\frac{11 \pi}{35}$ & 0.48627 & 0.49876 & 0.00381 & & 0.04266 & 2.972 \\
\hline & & & $\frac{12 \pi}{35}$ & 0.48636 & 0.49878 & 0.00374 & & 0.04245 & 3.056 \\
\hline & & & $\frac{13 \pi}{35}$ & 0.48644 & 0.49880 & 0.00368 & & 0.04228 & 3.120 \\
\hline & & & $\frac{2 \pi}{5}$ & 0.48650 & 0.49882 & 0.00363 & & 0.04214 & 3.168 \\
\hline & & & $\frac{16 \pi}{35}$ & 0.51340 & 0.50116 & 0.00357 & & 0.04198 & 3.223 \\
\hline & & & $\frac{17 \pi}{35}$ & 0.51337 & 0.50116 & 0.00356 & & 0.04195 & 3.233 \\
\hline & & & $\frac{18 \pi}{35}$ & 0.48666 & 0.49884 & 0.00356 & & 0.04196 & 3.230 \\
\hline & & & $\frac{19 \pi}{35}$ & 0.48667 & 0.49883 & 0.00358 & & 0.04200 & 3.21 \\
\hline & & & $\frac{3 \pi}{5}$ & 0.48665 & 0.49881 & 0.00365 & & 0.04219 & 3.150 \\
\hline & & & $\frac{22 \pi}{35}$ & 0.48663 & 0.49879 & 0.00370 & & 0.04234 & 3.096 \\
\hline & & & $\frac{23 \pi}{35}$ & 0.48659 & 0.49877 & 0.00376 & & 0.04253 & 3.025 \\
\hline & & & $\frac{24 \pi}{35}$ & 0.48654 & 0.49875 & 0.00384 & & 0.04276 & 2.935 \\
\hline
\end{tabular}

Table 9. Results for the quark mixing parameters obtained from the mixing patterns $V_{\mathrm{I}, 1}$ and $V_{\mathrm{I}, 2}$ with $n \leq 40$. We display the values of $\sin \theta_{i j}^{q}$ and $J_{\mathrm{CP}}^{q}$ which are compatible with experimental results for certain choices of the parameters $\theta_{u}, \theta_{d}, \varphi_{1}$ and $\varphi_{2}$. 


\begin{tabular}{|c|c|c|c|c|c|c|c|c|c|}
\hline & $n$ & $\varphi_{1}$ & $\varphi_{2}$ & $\theta_{u} / \pi$ & $\theta_{d} / \pi$ & $\sin \theta_{13}^{q}$ & $\sin \theta_{12}^{q}$ & $\sin \theta_{23}^{q}$ & $J_{\mathrm{CP}}^{q} / 10^{-5}$ \\
\hline \multirow{20}{*}{$V_{\mathrm{I}, 6}$} & \multirow{16}{*}{37} & \multirow{16}{*}{$\frac{18 \pi}{37}$} & $\frac{11 \pi}{37}$ & 0.99877 & 0.42769 & 0.00387 & 0.22512 & \multirow{16}{*}{0.04244} & 2.895 \\
\hline & & & $\frac{12 \pi}{37}$ & 0.99879 & 0.42769 & 0.00379 & 0.22505 & & 2.999 \\
\hline & & & $\frac{13 \pi}{37}$ & 0.99882 & 0.42774 & 0.00371 & 0.22499 & & 3.082 \\
\hline & & & $\frac{14 \pi}{37}$ & 0.99884 & 0.42776 & 0.00365 & 0.22495 & & 3.147 \\
\hline & & & $\frac{15 \pi}{37}$ & 0.00115 & 0.57222 & 0.00360 & 0.22491 & & 3.196 \\
\hline & & & $\frac{16 \pi}{37}$ & 0.00113 & 0.57221 & 0.00356 & 0.22488 & & 3.232 \\
\hline & & & $\frac{17 \pi}{37}$ & 0.00113 & 0.57220 & 0.00353 & 0.22487 & & 3.255 \\
\hline & & & $\frac{18 \pi}{37}$ & 0.00112 & 0.57219 & 0.00352 & 0.22486 & & 3.266 \\
\hline & & & $\frac{19 \pi}{37}$ & 0.00112 & 0.57219 & 0.00352 & 0.22486 & & 3.266 \\
\hline & & & $\frac{20 \pi}{37}$ & 0.00113 & 0.57219 & 0.00353 & 0.22487 & & 3.254 \\
\hline & & & $\frac{21 \pi}{37}$ & 0.00113 & 0.57219 & 0.00356 & 0.22488 & & 3.231 \\
\hline & & & $\frac{22 \pi}{37}$ & 0.00115 & 0.57220 & 0.00360 & 0.22491 & & 3.196 \\
\hline & & & $\frac{23 \pi}{37}$ & 0.99884 & 0.42780 & 0.00365 & 0.22495 & & 3.146 \\
\hline & & & $\frac{24 \pi}{37}$ & 0.99882 & 0.42779 & 0.00371 & 0.22499 & & 3.081 \\
\hline & & & $\frac{25 \pi}{37}$ & 0.99879 & 0.42777 & 0.00379 & 0.22505 & & 2.998 \\
\hline & & & $\frac{26 \pi}{37}$ & 0.99877 & 0.42775 & 0.00387 & 0.22512 & & 2.894 \\
\hline & \multirow{4}{*}{39} & \multirow{4}{*}{$\frac{19 \pi}{39}$} & $\frac{5 \pi}{13}$ & 0.99881 & 0.42774 & 0.00372 & 0.22500 & \multirow{4}{*}{0.04027} & 3.071 \\
\hline & & & $\frac{6 \pi}{13}$ & 0.00115 & 0.57222 & 0.00362 & 0.22493 & & 3.171 \\
\hline & & & $\frac{7 \pi}{13}$ & 0.00115 & 0.57221 & 0.00362 & 0.22493 & & 3.171 \\
\hline & & & $\frac{8 \pi}{13}$ & 0.99881 & 0.42778 & 0.00372 & 0.22500 & & 3.070 \\
\hline \multirow{3}{*}{$V_{\mathrm{I}, 8}$} & \multirow{3}{*}{37} & \multirow{3}{*}{$\frac{18 \pi}{37}$} & $\frac{3 \pi}{37}$ & 0.57236 & 0.00396 & 0.00373 & 0.22517 & 0.04406 & 2.914 \\
\hline & & & $\frac{4 \pi}{37}$ & 0.57223 & 0.00340 & 0.00348 & 0.22478 & 0.04363 & 3.309 \\
\hline & & & $\frac{5 \pi}{37}$ & 0.57217 & 0.00266 & 0.00397 & 0.22460 & 0.04307 & 3.193 \\
\hline
\end{tabular}

Table 10. Results for the quark mixing parameters obtained from the mixing patterns $V_{\mathrm{I}, 6}$ and $V_{\mathrm{I}, 8}$ with $n \leq 40$. We display the values of $\sin \theta_{i j}^{q}$ and $J_{\mathrm{CP}}^{q}$ which are compatible with experimental results for certain choices of the parameters $\theta_{u}, \theta_{d}, \varphi_{1}$ and $\varphi_{2}$. 
It is remarkable that the experimentally observed quark mixing angles and $\mathrm{CP}$ violation can be accommodated for the case of $\varphi_{1}=3 \pi / 7$ and $\varphi_{2}=3 \pi / 7$ (or $4 \pi / 7$ ), e.g.,

$$
\begin{aligned}
\theta_{u} & =0.48656 \pi(0.48666 \pi), & \theta_{d} & =0.49883 \pi(0.49882 \pi), \\
\sin \theta_{13}^{q} & =0.00359(0.00360), & \sin \theta_{12}^{q} & =0.22252(0.22252), \\
\sin \theta_{23}^{q} & =0.04204(0.04208), & J_{\mathrm{CP}}^{q} & =3.202 \times 10^{-5}\left(3.190 \times 10^{-5}\right) .
\end{aligned}
$$

The CKM element $V_{u s}$ is independent of the values of $\theta_{u, d}$ and it is given by

$$
\left|V_{u s}\right|=\cos \left(\frac{3 \pi}{7}\right)=\sin \left(\frac{\pi}{14}\right) \approx 0.2225
$$

This relation has been obtained in a study of the dihedral group $D_{14}[19,24,25]$. Since the dihedral group $D_{2 n}$ is a subgroup of $\Delta\left(6 n^{2}\right)$, the two generators of $D_{2 n}$ can be identified as the $\Delta\left(6 n^{2}\right)$ elements $b$ and $c d$, the realistic Cabibbo angle in eq. (4.12) is also reproduced from the flavor group $\Delta(294)$. We see that $\sin \theta_{23}^{q}, \sin \theta_{13}^{q}$ and $J_{\mathrm{CP}}^{q}$ are in the experimentally preferred ranges shown in eq. (4.2) while $\sin \theta_{12}^{q}$ is only about $1 \%$ smaller than its measured value. However, this could be quite easily reconciled with the experimental data in an explicit model with small corrections. Notice that all the measured values of the CKM mixing matrix elements can be reproduced in this approach, in particular the correct value of the quark $\mathrm{CP}$ violation phase can be obtained. On the other hand, in the paradigm of discrete flavor symmetry without $\mathrm{CP}$, only the realistic Cabibbo mixing angle can be predicted in terms of group theoretical quantities [19, 29], no matter whether the lefthanded quarks are assigned to an irreducible triplet representation of the flavor group, or to a reducible triplet which can decompose into a two-dimensional and a one-dimensional representation. Therefore we conclude that the flavor group $\Delta\left(6 \cdot 7^{2}\right)=\Delta(294)$ and CP symmetry provide a promising opportunity for model building to explain the quark flavor mixing and $\mathrm{CP}$ violation.

As shown in table 10, the mixing patterns $V_{\mathrm{I}, 6}$ and $V_{\mathrm{I}, 8}$ can give a good fit to the precisely measured CKM mixing matrix. For the mixing matrix $V_{\mathrm{I}, 6}$, the fixed element $\cos \varphi_{1}$ is the (23) entry, consequently we have the relation

$$
\left|V_{c b}\right|=\sin \theta_{23}^{q} \cos \theta_{13}^{q}=\left|\cos \varphi_{1}\right| .
$$

The experimental data on $V_{c b}$ can be accommodated for the values $\varphi_{1}=18 \pi / 37$ and $\varphi_{1}=19 \pi / 39$, accordingly the group index $n$ should be at least 37 and 39 respectively. For another mixing matrix $V_{\mathrm{I}, 8}$, the fixed element $\cos \varphi_{1}$ is the (32) entry, and the following sum rule is fulfilled

$$
\left|V_{t s}\right|=\left|\cos \theta_{12}^{q} \sin \theta_{23}^{q}+\sin \theta_{12}^{q} \cos \theta_{23}^{q} \sin \theta_{13}^{q} e^{i \delta_{\mathrm{CP}}^{q}}\right|=\left|\cos \varphi_{1}\right| .
$$

The parameter value $\varphi_{1}=18 \pi / 37$ leads to $\left|V_{t s}\right|=\cos (18 \pi / 37) \simeq 0.0424$ which is compatible with experimental data.

Moreover, the breaking of the $\Delta(294)$ flavor group and CP symmetry into distinct residual symmetries $Z_{2} \times C P$ in neutrino and charged lepton sectors can describe the 
experimentally measured values of the lepton mixing angles as well. Only the mixing patterns of case I and case II can be achieved from $\Delta(294)$ group since the group index $n$ has to be even for the other remaining cases. We find that the PMNS mixing matrices $U_{\mathrm{I}, 6}, U_{\mathrm{I}, 9}, U_{\mathrm{II}, 1}, U_{\mathrm{II}, 2}, U_{\mathrm{II}, 3}$ and $U_{\mathrm{II}, 4}$ can agree well with the experimental data for certain choices of $\theta_{\nu}$ and $\theta_{l}$. There are many possible phenomenologically viable cases and the corresponding predictions for the lepton mixing angles as well as CP phases from the $\chi^{2}$ analysis are shown in table 11 . We notice that the residual symmetries $Z_{2} \times C P$ of the lepton sector should be different from the quark sector, while the residual symmetry of either the neutrino or the charged lepton mass matrix can be identical with that of the up quark or down quark mass matrix. Moreover, we see that a variety of different values of the Dirac CP phase $\delta_{\mathrm{CP}}$ are allowed. In light of the weak evidence for $\delta_{\mathrm{CP}} \sim 3 \pi / 2$ [3-6], we would like to mention one interesting example of the mixing pattern $U_{\mathrm{I}, 6}$ with $\varphi_{1}=2 \pi / 7$ and $\varphi_{2}=3 \pi / 7$. The best fit values of the mixing parameters read

$$
\begin{aligned}
& \sin ^{2} \theta_{13}=0.0217, \quad \sin ^{2} \theta_{12}=0.306, \quad \sin ^{2} \theta_{23}=0.397, \\
& \left|\sin \delta_{\mathrm{CP}}\right|=0.946, \quad\left|\sin \alpha_{21}\right|=0.483, \quad\left|\sin \alpha_{31}\right|=0.350, \quad \chi_{\min }^{2}=4.320,
\end{aligned}
$$

which predicts approximately maximal $\delta_{\mathrm{CP}}$ and non-maximal atmospheric mixing angle $\theta_{23}$. If the Dirac phase $\delta_{\mathrm{CP}}$ is confirmed to be around $3 \pi / 2$ in upcoming long baseline experiments such as DUNE [76-79], T2HK [80, 81], T2HKK [82] and ESS $\nu$ SB [83, 84], a vast majority of solutions in table 11 would be excluded. Furthermore, we see that some cases have large differences in their predictions for $\theta_{12}, \theta_{23}$ and $\delta_{\mathrm{CP}}$ so that they might be distinguished from each other in future neutrino facilities. However, some other cases such as the mixing patterns $U_{\mathrm{II}, 1}$ with $\left(\varphi_{3}, \varphi_{4}\right)=(\pi / 7,0)$ and $\left(\varphi_{3}, \varphi_{4}\right)=(\pi / 7, \pi / 7)$, give similar predictions for lepton mixing angles and Dirac CP phase. Then a high-precision measurement of $\theta_{23}$ and $\delta_{\mathrm{CP}}$ would be needed, an option is to construct a more ambitious facility such as the neutrino factory.

Furthermore, we mention that the $\Delta(294)$ flavor group combined with CP symmetry can also give rise to phenomenologically viable lepton mixing pattern in the semidirect approach $[55,56]$ in which the original flavor and CP symmetries are broken to an abelian subgroup $G_{l}$ in the charged lepton sector and $Z_{2} \times C P$ in the neutrino sector. For instance, for the residual symmetries $G_{l}=Z_{3}^{a c^{s} d^{t}}, Z_{2}^{g_{\nu}}=Z_{2}^{b c^{x} d^{x}}, X_{\nu}=\left\{c^{\gamma} d^{-2 x-\gamma}, b c^{x+\gamma} d^{-x-\gamma}\right\}$ with $s, t, x, \gamma=0,1, \ldots, n-1$, the PMNS mixing matrix would be of the form [56]

$$
U_{\mathrm{Sd}}=\frac{1}{\sqrt{3}}\left(\begin{array}{ccc}
\sqrt{2} \sin \phi_{1} & e^{i \phi_{2}} \cos \theta-\sqrt{2} \sin \theta \cos \phi_{1} & e^{i \phi_{2}} \sin \theta+\sqrt{2} \cos \theta \cos \phi_{1} \\
\sqrt{2} \cos \left(\frac{\pi}{6}-\phi_{1}\right) & -e^{i \phi_{2}} \cos \theta-\sqrt{2} \sin \theta \sin \left(\frac{\pi}{6}-\phi_{1}\right) & -e^{i \phi_{2}} \sin \theta+\sqrt{2} \cos \theta \sin \left(\frac{\pi}{6}-\phi_{1}\right) \\
\sqrt{2} \cos \left(\frac{\pi}{6}+\phi_{1}\right) & e^{i \phi_{2}} \cos \theta+\sqrt{2} \sin \theta \sin \left(\frac{\pi}{6}+\phi_{1}\right) & e^{i \phi_{2}} \sin \theta-\sqrt{2} \cos \theta \sin \left(\frac{\pi}{6}+\phi_{1}\right)
\end{array}\right),
$$

up to possible permutations of rows and columns, the parameters $\phi_{1}$ and $\phi_{2}$ are determined by the residual symmetries as

$$
\phi_{1}=\frac{s-x}{n} \pi, \quad \phi_{2}=\frac{2 t-s-3(\gamma+x)}{n} \pi,
$$

which can take the following discrete values

$$
\phi_{1}(\bmod 2 \pi)=0, \frac{1}{n} \pi, \frac{2}{n} \pi, \ldots \frac{2 n-1}{n} \pi, \quad \phi_{2}(\bmod 2 \pi)=0, \frac{1}{n} \pi, \frac{2}{n} \pi, \ldots \frac{2 n-1}{n} \pi .
$$




\begin{tabular}{|c|c|c|c|c|c|c|c|c|c|c|c|}
\hline \multicolumn{12}{|c|}{ Case I for $n=7$} \\
\hline & $\varphi_{3}$ & $\varphi_{4}$ & $\theta_{l}^{\mathrm{bf}} / \pi$ & $\theta_{\nu}^{\mathrm{bf}} / \pi$ & $\chi_{\min }^{2}$ & $\sin ^{2} \theta_{13}$ & $\sin ^{2} \theta_{12}$ & $\sin ^{2} \theta_{23}$ & $\left|\sin \delta_{\mathrm{CP}}\right|$ & $\left|\sin \alpha_{21}\right|$ & $\left|\sin \alpha_{31}\right|$ \\
\hline \multirow{3}{*}{$U_{\mathrm{I}, 6}$} & \multirow{3}{*}{$\frac{2 \pi}{7}$} & $\frac{\pi}{7}$ & 0.940 & 0.652 & \multirow{3}{*}{4.320} & \multirow{3}{*}{0.0217} & \multirow{3}{*}{0.306} & \multirow{3}{*}{0.397} & 0.384 & 0.221 & 0.165 \\
\hline & & $\frac{2 \pi}{7}$ & 0.0603 & 0.338 & & & & & 0.721 & 0.392 & 0.290 \\
\hline & & $\frac{3 \pi}{7}$ & 0.0603 & 0.324 & & & & & 0.946 & 0.483 & 0.350 \\
\hline \multicolumn{12}{|c|}{ Case II fo } \\
\hline \multirow{8}{*}{$U_{\mathrm{II}, 1}$} & \multirow{2}{*}{0} & $\frac{\pi}{7}$ & 0.0921 & 0.0367 & 0.395 & 0.0217 & 0.312 & 0.433 & 0.170 & 0.0365 & 0.795 \\
\hline & & $\frac{2 \pi}{7}$ & 0.0859 & 0.0521 & 1.868 & 0.0219 & 0.321 & 0.432 & 0.449 & 0.0857 & 0.962 \\
\hline & \multirow{6}{*}{$\frac{\pi}{7}$} & 0 & 0.0926 & 0.0394 & 5.546 & 0.0219 & 0.331 & 0.419 & 0.662 & 0.327 & 0.206 \\
\hline & & $\frac{\pi}{7}$ & 0.100 & 0.0418 & 2.516 & 0.0218 & 0.322 & 0.422 & 0.525 & 0.393 & 0.914 \\
\hline & & $\frac{2 \pi}{7}$ & 0.107 & 0.0539 & 0.823 & 0.0217 & 0.314 & 0.429 & 0.324 & 0.471 & 0.862 \\
\hline & & $\frac{3 \pi}{7}$ & 0.102 & 0.0993 & 1.337 & 0.0219 & 0.319 & 0.440 & 0.330 & 0.561 & 0.0953 \\
\hline & & $\frac{4 \pi}{7}$ & 0.270 & 0.320 & 3.561 & 0.0218 & 0.309 & 0.402 & 0.471 & 0.227 & 0.898 \\
\hline & & $\frac{5 \pi}{7}$ & 0.278 & 0.386 & 1.145 & 0.0218 & 0.318 & 0.434 & 0.359 & 0.627 & 0.657 \\
\hline \multirow{16}{*}{$U_{\mathrm{II}, 2}$} & \multirow{2}{*}{0} & $\frac{\pi}{7}$ & 0.920 & 0.987 & 14.119 & 0.0218 & 0.321 & 0.536 & 0.0942 & 0.0074 & 0.777 \\
\hline & & $\frac{2 \pi}{7}$ & 0.922 & 0.982 & 14.968 & 0.0219 & 0.326 & 0.535 & 0.238 & 0.0184 & 0.979 \\
\hline & \multirow{5}{*}{$\frac{\pi}{7}$} & 0 & 0.914 & 0.982 & 19.130 & 0.0219 & 0.326 & 0.550 & 0.558 & 0.351 & 0.217 \\
\hline & & $\frac{\pi}{7}$ & 0.912 & 0.982 & 16.981 & 0.0218 & 0.321 & 0.547 & 0.444 & 0.349 & 0.896 \\
\hline & & $\frac{2 \pi}{7}$ & 0.911 & 0.977 & 15.162 & 0.0218 & 0.319 & 0.542 & 0.282 & 0.340 & 0.911 \\
\hline & & $\frac{3 \pi}{7}$ & 0.917 & 0.962 & 15.342 & 0.0220 & 0.333 & 0.527 & 0.0863 & 0.286 & 0.296 \\
\hline & & $\frac{6 \pi}{7}$ & 0.919 & 0.0193 & 23.256 & 0.0220 & 0.337 & 0.550 & 0.659 & 0.338 & 0.633 \\
\hline & \multirow{5}{*}{$\frac{2 \pi}{7}$} & 0 & 0.462 & 0.627 & 17.931 & 0.0219 & 0.313 & 0.554 & 0.471 & 0.300 & 0.187 \\
\hline & & $\frac{\pi}{7}$ & 0.887 & 0.958 & 36.203 & 0.0219 & 0.334 & 0.591 & 0.920 & 0.751 & 0.990 \\
\hline & & $\frac{2 \pi}{7}$ & 0.879 & 0.953 & 24.739 & 0.0217 & 0.321 & 0.571 & 0.747 & 0.767 & 0.727 \\
\hline & & $\frac{3 \pi}{7}$ & 0.881 & 0.915 & 14.053 & 0.0219 & 0.323 & 0.534 & 0.00242 & 0.705 & 0.0238 \\
\hline & & $\frac{6 \pi}{7}$ & 0.452 & 0.369 & 15.617 & 0.0218 & 0.328 & 0.536 & 0.300 & 0.881 & 0.726 \\
\hline & \multirow{4}{*}{$\frac{3 \pi}{7}$} & 0 & 0.458 & 0.643 & 33.524 & 0.022 & 0.281 & 0.587 & 0.636 & 0.432 & 0.289 \\
\hline & & $\frac{2 \pi}{7}$ & 0.734 & 0.735 & 34.547 & 0.0218 & 0.291 & 0.596 & 0.788 & 0.580 & 0.560 \\
\hline & & $\frac{5 \pi}{7}$ & 0.383 & 0.326 & 18.918 & 0.0219 & 0.342 & 0.525 & 0.326 & 0.630 & 0.989 \\
\hline & & $\frac{6 \pi}{7}$ & 0.421 & 0.362 & 16.210 & 0.0218 & 0.309 & 0.549 & 0.294 & 0.996 & 0.871 \\
\hline
\end{tabular}

Table 11. Results of the lepton mixing parameters for the viable cases obtained from the $\Delta(294)$ flavor group. All values of $\sin ^{2} \theta_{i j},\left|\sin \delta_{\mathrm{CP}}\right|,\left|\sin \alpha_{21}\right|$ and $\left|\sin \alpha_{31}\right|$ are obtained at the best fitting points $\left(\theta_{l}, \theta_{\nu}\right)=\left(\theta_{l}^{\mathrm{bf}}, \theta_{\nu}^{\mathrm{bf}}\right)$ under the assumption of $\mathrm{NH}$ neutrino spectrum, and similar results are obtained for IH spectrum. We don't display the cases which can accommodate the measured values of the lepton mixing angles yet predict trivial CP phases.

We see that one column is fixed to be $\left(\sqrt{2} \sin \phi_{1}, \sqrt{2} \cos \left(\pi / 6-\phi_{1}\right), \sqrt{2} \cos \left(\pi / 6+\phi_{1}\right)\right)^{T} / \sqrt{3}$ by the group theory. For the case of $n=7$, it has to be identified as the first column of the mixing matrix in order to be compatible with experimental data on lepton mixing angles. Subsequently considering all possible values of $\phi_{1}$ and $\phi_{2}$, we find that two mixing patterns resulting from the row permutations are viable, i.e.

$$
U_{\mathrm{Sd}, 1}=P_{23} P_{12} U_{\mathrm{Sd}}, \quad U_{\mathrm{Sd}, 2}=P_{12} U_{\mathrm{Sd}} .
$$

Since $U_{\mathrm{Sd}, 2}$ can be obtained by permuting the second and the third rows of $U_{\mathrm{Sd}, 1}$, we will 
only show the predictions of $U_{\mathrm{Sd}, 1}$ in the following. The corresponding results for the mixing parameters and the best fit value $\theta^{\text {bf }}$ of the free parameter $\theta$ are summarized in table 12 . Notice that approximately maximal Dirac phase together with nearly maximal $\theta_{23}$ can be achieved. For example, for the mixing matrix $U_{\mathrm{Sd}, 1}$ with $\phi_{1}=\pi / 7$ and $\phi_{2}=3 \pi / 7$, we can derive the following sum rule relating the atmospheric mixing angle to the reactor one

$$
\sin ^{2} \theta_{23} \simeq \frac{\sqrt{3} \sin \frac{2 \pi}{7}}{3 \cos \frac{\pi}{21}} \mp \frac{\sqrt{2} \sin \frac{\pi}{7}}{2 \cos ^{2} \frac{\pi}{21}} \sin \theta_{13},
$$

where the sign "-" is for $0<\theta<\pi / 2$ and "+" for $\pi / 2<\theta<\pi$. Inserting the measured value $\sin ^{2} \theta_{13} \simeq 0.02166$ from the global fit [8], we find $\sin ^{2} \theta_{23} \simeq 0.410$ or 0.503 which are in the experimentally preferred $3 \sigma$ range [8]. Furthermore, we can obtain an approximate relation of the Dirac $\mathrm{CP}$ phase and $\sin \theta_{13}$,

$$
\sin \delta_{\mathrm{CP}} \simeq \frac{\left(\sqrt{2} \sin \frac{\pi}{42} \sin \frac{\pi}{14} \pm \sqrt{3 \sin ^{2} \theta_{13}-2 \sin ^{2} \frac{\pi}{42}}\right) \cos ^{2} \frac{\pi}{14}}{\sqrt{3} \sin \theta_{13}} .
$$

We get $\left|\sin \delta_{\mathrm{CP}}\right| \simeq 0.953$ or 0.777 for $\sin ^{2} \theta_{13} \simeq 0.02166$. The above estimates are consistent with the results of the $\chi^{2}$ analysis, see table 12 . The $\chi^{2}$ function reaches a global minimum at the best fit value $\theta^{\text {bf }} \simeq 0.0829 \pi$ for $\mathrm{NH}$, accordingly the mixing parameters are given by

$$
\begin{aligned}
& \sin ^{2} \theta_{13}=0.0217, \quad \sin ^{2} \theta_{12}=0.322, \quad \sin ^{2} \theta_{23}=0.413, \\
& \left|\sin \delta_{\mathrm{CP}}\right|=0.971, \quad\left|\sin \alpha_{21}\right|=0.482, \quad\left|\sin \alpha_{31}\right|=0.129, \quad \chi_{\min }^{2}=3.656 .
\end{aligned}
$$

We conclude that the flavor group $\Delta(294)$ and CP symmetry are good starting point to build models which can simultaneously explain lepton and quark flavor mixing and CP violation. Guided by the analysis of this paper, we could introduce appropriate flavon fields to break $\Delta(294)$ and CP symmetries into $Z_{2} \times C P$ subgroups in the up quark, down quark and neutrino sectors while the residual symmetry of the charged lepton mass term can be either $Z_{3}$ or $Z_{2} \times C P$. Accordingly the whole quark and lepton flavor mixing structures are described in terms of only three or four free parameters.

\section{$5 \quad$ Summary and conclusions}

In the most widely discussed scenario involving discrete flavor symmetry and CP symmetry, it is usually assumed that the original flavor and CP symmetries are broken to an abelian subgroup and $Z_{2} \times C P$ in the charged lepton and neutrino sectors respectively. In this work we study the case that the flavor and CP symmetries are broken to $Z_{2} \times C P$ in both neutrino and charged lepton sectors. The consequences for the prediction of the lepton mixing parameters are discussed. In this setup, at least one element of the lepton mixing matrix is fixed to be certain constant, all lepton mixing angles and all $\mathrm{CP}$ violation phases (both Dirac and Majorana phases) depend on two free parameters $\theta_{l}$ and $\theta_{\nu}$ which vary between 0 and $\pi$.

In this paper we have derived the predictions for lepton mixing in a class of models based on $\Delta\left(6 n^{2}\right)$ flavor group combined with CP symmetry. We have considered all possible 


\begin{tabular}{|c|c|c|c|c|c|c|c|c|c|c|}
\hline \multicolumn{11}{|c|}{ Semidirect approach for $n=7$} \\
\hline \multicolumn{11}{|c|}{$\sin ^{2} \theta_{13}=\frac{1}{3}\left[1-\sqrt{2} \sin 2 \theta \sin \left(\frac{\pi}{6}-\phi_{1}\right) \cos \phi_{2}-\cos ^{2} \theta \cos \left(\frac{\pi}{3}-2 \phi_{1}\right)\right]$} \\
\hline \multicolumn{11}{|c|}{$\sin ^{2} \theta_{12}=\frac{1+\sqrt{2} \sin 2 \theta \sin \left(\frac{\pi}{6}-\phi_{1}\right) \cos \phi_{2}-\sin ^{2} \theta \cos \left(\frac{\pi}{3}-2 \phi_{1}\right)}{2+\sqrt{2} \sin 2 \theta \sin \left(\frac{\pi}{6}-\phi_{1}\right) \cos \phi_{2}+\cos ^{2} \theta \cos \left(\frac{\pi}{3}-2 \phi_{1}\right)}$} \\
\hline \multicolumn{11}{|c|}{$\sin ^{2} \theta_{23}=\frac{1-\sqrt{2} \sin 2 \theta \sin \left(\frac{\pi}{6}+\phi_{1}\right) \cos \phi_{2}-\cos ^{2} \theta \cos \left(\frac{\pi}{3}+2 \phi_{1}\right)}{2+\sqrt{2} \sin 2 \theta \sin \left(\frac{\pi}{6}-\phi_{1}\right) \cos \phi_{2}+\cos ^{2} \theta \cos \left(\frac{\pi}{3}-2 \phi_{1}\right)}$ for $U_{\mathrm{Sd}, 1}$} \\
\hline \multicolumn{11}{|c|}{$\sin ^{2} \theta_{23}=\frac{1+\sqrt{2} \sin 2 \theta \cos \phi_{1} \cos \phi_{2}+\cos ^{2} \theta \cos 2 \phi_{1}}{2+\sqrt{2} \sin 2 \theta \sin \left(\frac{\pi}{6}-\phi_{1}\right) \cos \phi_{2}+\cos ^{2} \theta \cos \left(\frac{\pi}{3}-2 \phi_{1}\right)}$ for $U_{\mathrm{Sd}, 2}$} \\
\hline \multicolumn{11}{|c|}{$\left|J_{\mathrm{CP}}\right|=\frac{1}{6 \sqrt{6}}\left|\sin 2 \theta \sin 3 \phi_{1} \sin \phi_{2}\right|$} \\
\hline \multicolumn{11}{|c|}{$\left|I_{1}\right|=\frac{4}{9}\left|\cos \theta \cos ^{2}\left(\frac{\pi}{6}-\phi_{1}\right) \sin \phi_{2}\left[\cos \theta \cos \phi_{2}+\sqrt{2} \sin \theta \sin \left(\frac{\pi}{6}-\phi_{1}\right)\right]\right|$} \\
\hline \multicolumn{11}{|c|}{$\left|I_{2}\right|=\frac{4}{9}\left|\sin \theta \cos ^{2}\left(\frac{\pi}{6}-\phi_{1}\right) \sin \phi_{2}\left[\sin \theta \cos \phi_{2}-\sqrt{2} \cos \theta \sin \left(\frac{\pi}{6}-\phi_{1}\right)\right]\right|$} \\
\hline & $\phi_{1}$ & $\phi_{2}$ & $\theta^{\mathrm{bf}} / \pi$ & $\chi_{\min }^{2}$ & $\sin ^{2} \theta_{13}$ & $\sin ^{2} \theta_{12}$ & $\sin ^{2} \theta_{23}$ & $\left|\sin \delta_{\mathrm{CP}}\right|$ & $\left|\sin \alpha_{21}\right|$ & $\left|\sin \alpha_{31}\right|$ \\
\hline \multirow{3}{*}{$U_{\mathrm{Sd}, 1}$} & \multirow{3}{*}{$\frac{\pi}{7}$} & $\frac{\pi}{7}$ & 0.951 & 25.538 & 0.0212 & 0.323 & 0.571 & 0.266 & 0.791 & 0.00146 \\
\hline & & $\frac{2 \pi}{7}$ & 0.944 & 17.379 & 0.0214 & 0.323 & 0.547 & 0.537 & 0.968 & 0.00828 \\
\hline & & $\frac{3 \pi}{7}$ & 0.0829 & 3.656 & 0.0217 & 0.322 & 0.413 & 0.971 & 0.482 & 0.129 \\
\hline
\end{tabular}

Table 12. Results of the lepton mixing parameters for the viable cases obtained from the $\Delta(294)$ flavor group in the semidirect approach [56]. All values of $\sin ^{2} \theta_{i j},\left|\sin \delta_{\mathrm{CP}}\right|,\left|\sin \alpha_{21}\right|$ and $\left|\sin \alpha_{31}\right|$ are obtained at the best fitting points $\theta=\theta^{\text {bf }}$ under the assumption of $\mathrm{NH}$ neutrino spectrum, and similar results are obtained for IH spectrum. Since the PMNS matrix $U_{\mathrm{Sd}}$ has the property $U_{\mathrm{Sd}}\left(\phi_{1}, \pi-\phi_{2}, \theta\right)=U_{\mathrm{Sd}}^{*}\left(\phi_{1}, \phi_{2}, \pi-\theta\right) \operatorname{diag}(1,1,-1)$, hence we only show the results for $0 \leq \phi_{2}<\pi / 2$. We don't display the cases which can accommodate the measured values of the lepton mixing angles yet predict trivial $\mathrm{CP}$ phases.

choices of residual subgroups of the structure $Z_{2} \times C P$. We find that the residual symmetries enforce one element of the lepton mixing matrix to be $0,1,1 / 2,1 / \sqrt{2}$ and $\cos \varphi_{1}$ where the parameter $\varphi_{1}$ given by eq. (3.9) is related to the choice of residual $Z_{2}$ flavor symmetry. Obviously the cases with the entry equal to 0 or 1 are excluded by the measurement of the reactor angle $\theta_{13}$. It turns out that only four possible combinations of residual symmetries can lead to phenomenologically viable mixing patterns. We perform an analytical study of all possible mixing patterns, and the permutations of rows and columns of the mixing matrix are taken into account. The lepton mixing angles and Dirac CP phase are strongly correlated in each of these cases, a mixing sum rule is satisfied and it can be tested in future neutrino oscillation facilities. Furthermore, we perform a numerical analysis for small values of the group index $n$ which can admit a good agreement with experimental data. The resulting predictions for the effective Majorana mass in neutrinoless double beta decay are studied. We show that in all cases it is sufficient to considered the $\Delta\left(6 n^{2}\right)$ groups with index $n \leq 4$. The increased precision on measurements of $\theta_{12}, \theta_{23}$ and $\delta_{\mathrm{CP}}$ from next generation long baseline neutrino oscillation experiments could help us to test the predictions reached in this work and find out the smallest $\Delta\left(6 n^{2}\right)$ flavor group together with the symmetry breaking patterns which can describe data best.

There are many attempts to produce the extremely hierarchical structure of the quark CKM mixing matrix from discrete flavor symmetry. It is found that no finite group can 
predict all mixing angles and CP phase of the CKM matrix and only phenomenologically acceptable Cabibbo angle can be generated $[19,29]$. In the present work, we investigate whether it is possible to derive quark mixing in an analogous way as we do for the lepton mixing. It is assumed that two distinct $Z_{2} \times C P$ residual symmetries are separately preserved by the up and down quark mass terms. As a consequence, all the three quark mixing angles and $\mathrm{CP}$ violation phase are expressed in terms of two free real parameters $\theta_{u}$ and $\theta_{d}$ which can take values between 0 and $\pi$. As an example, we consider the series of flavor group $\Delta\left(6 n^{2}\right)$ combined with CP symmetry. We find that the quark mixing pattern arising from the residual symmetries $Z_{2}^{g_{u}}=Z_{2}^{b c^{x} d^{x}}, X_{u}=\left\{c^{\gamma} d^{-2 x-\gamma}, b c^{x+\gamma} d^{-x-\gamma}\right\}, Z_{2}^{g_{d}}=Z_{2}^{b c^{y} d^{y}}$ and $X_{d}=\left\{c^{\delta} d^{-2 y-\delta}, b c^{y+\delta} d^{-y-\delta}\right\}$ with $x, y, \gamma, \delta=0,1, \ldots, n-1$ can be compatible with the experimental data on CKM mixing matrix. We perform a numerical analysis for the groups with the index $n \leq 40$, and find out all the viable mixing patters. The corresponding predictions for the quark mixing angles and $\mathrm{CP}$ invariant are summarized in table 9 and table 10. The smallest value of the group index $n$ which allows a good fit to the experimental data is $n=7$.

Furthermore, we find that a common flavor group such as $\Delta\left(6 \cdot 7^{2}\right)=\Delta(294)$ can simultaneously describe the experimentally measured values of the quark and lepton mixing matrices if the parent flavor and CP symmetries are broken down to $Z_{2} \times C P$ in all the neutrino, charged lepton, up quark and down quark sectors, or alternatively the residual symmetry of the charged lepton mass term is $Z_{3}$ instead of $Z_{2} \times C P$. In our approach, the drastically different quark and lepton flavor mixing structures originate from the mismatch of different residual symmetries. The symmetry breaking pattern indicated here provides a new starting point for flavor model building. In concrete models the residual symmetry is generally achieved via spontaneous symmetry breaking of flavon fields in some vacuum alignment configurations. It is interesting to construct an actual model in which the desired breaking pattern is dynamically realized. We see that the representative values of the free parameters $\theta_{l}, \theta_{\nu}, \theta_{u}$ and $\theta_{d}$ are generically of order one, consequently we expect that the desired values of these free parameters can be achieved in an explicit model without too much fine-tuning, since the resulting lepton mixing angles are still compatible with the experimental data if $\theta_{l, \nu}$ vary within $5 \%$ or so of their best fit values shown in various tables, and the precisely measured CKM matrix can also be accommodated for $\theta_{u, d}$ within $1 \%$ of their representative values. In addition, we expect such model could reproduce the huge mass hierarchies among quarks and leptons with the help of additional symmetry such as $Z_{n_{1}} \times Z_{n_{2}} \times \ldots$ in the Froggatt-Nielsen scenario [102]. There have been several previous attempts to predict the CKM and PMNS mixing matrices from a common discrete flavor group [103, 104], the CP violation in CKM matrix was obtained by producing some special textures of the up and down quark mass matrices with the help of discrete vacuum alignment method.

In this paper we have focused on the series of the flavor group $\Delta\left(6 n^{2}\right)$. The other two group series $\Delta\left(3 n^{2}\right)[55,57]$ and $D_{9 n, 3 n}^{(1)} \cong\left(Z_{9 n} \times Z_{3 n}\right) \rtimes S_{3}$ [58] are also frequently employed as flavor symmetry. Because $\Delta\left(3 n^{2}\right)$ is a subgroup of $\Delta\left(6 n^{2}\right)$ and the relation $\Delta\left(6(3 n)^{2}\right) \subset D_{9 n, 3 n}^{(1)} \subset \Delta\left(6(9 n)^{2}\right)$ holds true, $\Delta\left(3 n^{2}\right)$ and $D_{9 n, 3 n}^{(1)}$ should not give new additional results within the present framework. Inspired by the capability of explaining 
the $\mathrm{CP}$ violation in the CKM mixing matrix, it is also interesting to explore whether the flavor and CP symmetries are helpful to solve the strong CP problem.

\section{Acknowledgments}

G.-J. D. and J.-N. L. acknowledge the support of the National Natural Science Foundation of China under Grant No. 11522546. C.-C. L. is supported by CPSF-CAS Joint Foundation for Excellent Postdoctoral Fellows No. 2017L-H0003 and China Postdoctoral Science Foundation Grant No. 2017M620258.

Open Access. This article is distributed under the terms of the Creative Commons Attribution License (CC-BY 4.0), which permits any use, distribution and reproduction in any medium, provided the original author(s) and source are credited.

\section{References}

[1] Particle Data Group collaboration, C. Patrignani et al., Review of Particle Physics, Chin. Phys. C 40 (2016) 100001 [inSPIRE].

[2] M. Kobayashi and T. Maskawa, CP Violation in the Renormalizable Theory of Weak Interaction, Prog. Theor. Phys. 49 (1973) 652 [INSPIRE].

[3] K. Iwamoto on behalf of T2K collaboration, Recent results from T2K and future prospects, talk given at the 38th International Conference on High Energy Physics (ICHEP 2016), Chicago, Illinois, U.S.A., 3-10 August 2016, PoS(ICHEP2016) 517 [http://indico.cern.ch/event/432527/contributions/2143636/] [INSPIRE].

[4] J. Bian for the NO $\nu \mathrm{A}$ collaboration, Recent Results of Electron-Neutrino Appearance

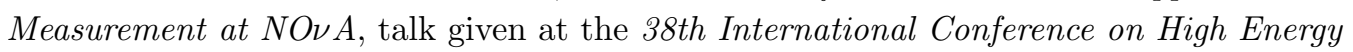
Physics, Chicago, Illinois, U.S.A., 3-10 August 2016, PoS(ICHEP2016) 516 [http://indico.cern.ch/event/432527/contributions/2144798/] [INSPIRE].

[5] T2K collaboration, K. Abe et al., Combined Analysis of Neutrino and Antineutrino Oscillations at T2K, Phys. Rev. Lett. 118 (2017) 151801 [arXiv:1701.00432] [INSPIRE].

[6] NOvA collaboration, P. Adamson et al., Constraints on Oscillation Parameters from $\nu_{e}$ Appearance and $\nu_{\mu}$ Disappearance in NOvA, Phys. Rev. Lett. 118 (2017) 231801 [arXiv:1703.03328] [INSPIRE].

[7] D.V. Forero, M. Tortola and J.W.F. Valle, Neutrino oscillations refitted, Phys. Rev. D 90 (2014) 093006 [arXiv: 1405.7540] [INSPIRE].

[8] I. Esteban, M.C. Gonzalez-Garcia, M. Maltoni, I. Martinez-Soler and T. Schwetz, Updated fit to three neutrino mixing: exploring the accelerator-reactor complementarity, JHEP 01 (2017) 087 [arXiv: 1611.01514] [INSPIRE].

[9] F. Capozzi, E. Di Valentino, E. Lisi, A. Marrone, A. Melchiorri and A. Palazzo, Global constraints on absolute neutrino masses and their ordering, Phys. Rev. D 95 (2017) 096014 [arXiv: 1703.04471] [INSPIRE].

[10] G. Altarelli and F. Feruglio, Discrete Flavor Symmetries and Models of Neutrino Mixing, Rev. Mod. Phys. 82 (2010) 2701 [arXiv:1002.0211] [INSPIRE]. 
[11] H. Ishimori, T. Kobayashi, H. Ohki, Y. Shimizu, H. Okada and M. Tanimoto, Non-Abelian Discrete Symmetries in Particle Physics, Prog. Theor. Phys. Suppl. 183 (2010) 1 [arXiv: 1003.3552] [INSPIRE].

[12] S.F. King and C. Luhn, Neutrino Mass and Mixing with Discrete Symmetry, Rept. Prog. Phys. 76 (2013) 056201 [arXiv: 1301.1340] [INSPIRE].

[13] S.F. King, A. Merle, S. Morisi, Y. Shimizu and M. Tanimoto, Neutrino Mass and Mixing: from Theory to Experiment, New J. Phys. 16 (2014) 045018 [arXiv:1402.4271] [InSPIRE].

[14] S.F. King, Models of Neutrino Mass, Mixing and CP-violation, J. Phys. G 42 (2015) 123001 [arXiv: 1510.02091] [INSPIRE].

[15] M. Holthausen, K.S. Lim and M. Lindner, Lepton Mixing Patterns from a Scan of Finite Discrete Groups, Phys. Lett. B 721 (2013) 61 [arXiv:1212.2411] [INSPIRE].

[16] S.F. King, T. Neder and A.J. Stuart, Lepton mixing predictions from $\Delta\left(6 n^{2}\right)$ family Symmetry, Phys. Lett. B 726 (2013) 312 [arXiv: 1305.3200] [INSPIRE].

[17] R.M. Fonseca and W. Grimus, Classification of lepton mixing matrices from finite residual symmetries, JHEP 09 (2014) 033 [arXiv: 1405.3678] [INSPIRE].

[18] J. Talbert, [Re]constructing Finite Flavour Groups: Horizontal Symmetry Scans from the Bottom-Up, JHEP 12 (2014) 058 [arXiv:1409.7310] [INSPIRE].

[19] C.-Y. Yao and G.-J. Ding, Lepton and Quark Mixing Patterns from Finite Flavor Symmetries, Phys. Rev. D 92 (2015) 096010 [arXiv:1505.03798] [InSPIRE].

[20] S.-F. Ge, D.A. Dicus and W.W. Repko, $Z_{2}$ Symmetry Prediction for the Leptonic Dirac CP Phase, Phys. Lett. B 702 (2011) 220 [arXiv:1104.0602] [INSPIRE].

[21] D. Hernandez and A.Y. Smirnov, Lepton mixing and discrete symmetries, Phys. Rev. D 86 (2012) 053014 [arXiv: 1204.0445] [INSPIRE].

[22] D. Hernandez and A.Y. Smirnov, Discrete symmetries and model-independent patterns of lepton mixing, Phys. Rev. D 87 (2013) 053005 [arXiv: 1212.2149] [INSPIRE].

[23] I. Girardi, S.T. Petcov, A.J. Stuart and A.V. Titov, Leptonic Dirac CP-violation Predictions from Residual Discrete Symmetries, Nucl. Phys. B 902 (2016) 1 [arXiv: 1509.02502] [INSPIRE].

[24] C.S. Lam, Symmetry of Lepton Mixing, Phys. Lett. B 656 (2007) 193 [arXiv:0708.3665] [INSPIRE].

[25] A. Blum, C. Hagedorn and M. Lindner, Fermion Masses and Mixings from Dihedral Flavor Symmetries with Preserved Subgroups, Phys. Rev. D 77 (2008) 076004 [arXiv:0709.3450] [INSPIRE].

[26] R. de Adelhart Toorop, F. Feruglio and C. Hagedorn, Finite Modular Groups and Lepton Mixing, Nucl. Phys. B 858 (2012) 437 [arXiv:1112.1340] [INSPIRE].

[27] M. Holthausen and K.S. Lim, Quark and Leptonic Mixing Patterns from the Breakdown of a Common Discrete Flavor Symmetry, Phys. Rev. D 88 (2013) 033018 [arXiv:1306.4356] [INSPIRE].

[28] T. Araki, H. Ishida, H. Ishimori, T. Kobayashi and A. Ogasahara, CKM matrix and flavor symmetries, Phys. Rev. D 88 (2013) 096002 [arXiv:1309.4217] [INSPIRE]. 
[29] I. de Medeiros Varzielas, R.W. Rasmussen and J. Talbert, Bottom-Up Discrete Symmetries for Cabibbo Mixing, Int. J. Mod. Phys. A 32 (2017) 1750047 [arXiv:1605.03581] [INSPIRE].

[30] F. Feruglio, C. Hagedorn and R. Ziegler, Lepton Mixing Parameters from Discrete and CP Symmetries, JHEP 07 (2013) 027 [arXiv:1211.5560] [INSPIRE].

[31] P. Chen, C.-C. Li and G.-J. Ding, Lepton Flavor Mixing and CP Symmetry, Phys. Rev. D 91 (2015) 033003 [arXiv:1412.8352] [InSPIRE].

[32] L.L. Everett, T. Garon and A.J. Stuart, A Bottom-Up Approach to Lepton Flavor and CP Symmetries, JHEP 04 (2015) 069 [arXiv:1501.04336] [INSPIRE].

[33] P. Chen, C.-Y. Yao and G.-J. Ding, Neutrino Mixing from CP Symmetry, Phys. Rev. D 92 (2015) 073002 [arXiv: 1507.03419] [inSPIRE].

[34] L.L. Everett and A.J. Stuart, Lepton Sector Phases and Their Roles in Flavor and Generalized CP Symmetries, Phys. Rev. D 96 (2017) 035030 [arXiv:1611.03020] [INSPIRE].

[35] W. Grimus and M.N. Rebelo, Automorphisms in gauge theories and the definition of CP and P, Phys. Rept. 281 (1997) 239 [hep-ph/9506272] [INSPIRE].

[36] M. Holthausen, M. Lindner and M.A. Schmidt, CP and Discrete Flavour Symmetries, JHEP 04 (2013) 122 [arXiv:1211.6953] [InSPIRE].

[37] M.-C. Chen, M. Fallbacher, K.T. Mahanthappa, M. Ratz and A. Trautner, CP Violation from Finite Groups, Nucl. Phys. B 883 (2014) 267 [arXiv:1402.0507] [INSPIRE].

[38] G.-J. Ding, S.F. King and A.J. Stuart, Generalised CP and $A_{4}$ Family Symmetry, JHEP 12 (2013) 006 [arXiv:1307.4212] [INSPIRE].

[39] C.C. Nishi, New and trivial CP symmetry for extended $A_{4}$ flavor, Phys. Rev. D 93 (2016) 093009 [arXiv: 1601.00977] [INSPIRE].

[40] C.-C. Li, J.-N. Lu and G.-J. Ding, $A_{4}$ and CP symmetry and a model with maximal CP-violation, Nucl. Phys. B 913 (2016) 110 [arXiv:1608.01860] [INSPIRE].

[41] G.-J. Ding, S.F. King, C. Luhn and A.J. Stuart, Spontaneous CP-violation from vacuum alignment in $S_{4}$ models of leptons, JHEP 05 (2013) 084 [arXiv:1303.6180] [INSPIRE].

[42] F. Feruglio, C. Hagedorn and R. Ziegler, A realistic pattern of lepton mixing and masses from $S_{4}$ and CP, Eur. Phys. J. C $\mathbf{7 4}$ (2014) 2753 [arXiv:1303.7178] [InSPIRE].

[43] C. Luhn, Trimaximal $T M_{1}$ neutrino mixing in $S_{4}$ with spontaneous CP-violation, Nucl. Phys. B 875 (2013) 80 [arXiv:1306.2358] [InSPIRE].

[44] C.-C. Li and G.-J. Ding, Generalised $C P$ and trimaximal $T M_{1}$ lepton mixing in $S_{4}$ family symmetry, Nucl. Phys. B 881 (2014) 206 [arXiv:1312.4401] [INSPIRE].

[45] C.-C. Li and G.-J. Ding, Deviation from bimaximal mixing and leptonic CP phases in $S_{4}$ family symmetry and generalized CP, JHEP 08 (2015) 017 [arXiv:1408.0785] [INSPIRE].

[46] G.-J. Ding and Y.-L. Zhou, Predicting lepton flavor mixing from $\Delta(48)$ and generalized $C P$ symmetries, Chin. Phys. C 39 (2015) 021001 [arXiv:1312.5222] [INSPIRE].

[47] G.-J. Ding and Y.-L. Zhou, Lepton mixing parameters from $\Delta(48)$ family symmetry and generalised CP, JHEP 06 (2014) 023 [arXiv: 1404.0592] [INSPIRE]. 
[48] G.-J. Ding and S.F. King, Generalized $C P$ and $\Delta(96)$ family symmetry, Phys. Rev. D 89 (2014) 093020 [arXiv: 1403.5846] [INSPIRE].

[49] C.-C. Li and G.-J. Ding, Lepton Mixing in $A_{5}$ Family Symmetry and Generalized CP, JHEP 05 (2015) 100 [arXiv:1503.03711] [INSPIRE].

[50] A. Di Iura, C. Hagedorn and D. Meloni, Lepton mixing from the interplay of the alternating group $A_{5}$ and CP, JHEP 08 (2015) 037 [arXiv: 1503.04140] [InSPIRE].

[51] P. Ballett, S. Pascoli and J. Turner, Mixing angle and phase correlations from $A_{5}$ with generalized CP and their prospects for discovery, Phys. Rev. D 92 (2015) 093008 [arXiv: 1503.07543] [INSPIRE].

[52] G.C. Branco, I. de Medeiros Varzielas and S.F. King, Invariant approach to CP in family symmetry models, Phys. Rev. D 92 (2015) 036007 [arXiv: 1502.03105] [INSPIRE].

[53] G.C. Branco, I. de Medeiros Varzielas and S.F. King, Invariant approach to $\mathcal{C P}$ in unbroken $\Delta(27)$, Nucl. Phys. B 899 (2015) 14 [arXiv:1505.06165] [INSPIRE].

[54] S.F. King and T. Neder, Lepton mixing predictions including Majorana phases from $\Delta\left(6 n^{2}\right)$ flavour symmetry and generalised CP, Phys. Lett. B 736 (2014) 308 [arXiv:1403.1758] [INSPIRE].

[55] C. Hagedorn, A. Meroni and E. Molinaro, Lepton mixing from $\Delta\left(3 n^{2}\right)$ and $\Delta\left(6 n^{2}\right)$ and $C P$, Nucl. Phys. B 891 (2015) 499 [arXiv: 1408.7118] [INSPIRE].

[56] G.-J. Ding, S.F. King and T. Neder, Generalised $C P$ and $\Delta\left(6 n^{2}\right)$ family symmetry in semi-direct models of leptons, JHEP 12 (2014) 007 [arXiv: 1409.8005] [INSPIRE].

[57] G.-J. Ding and S.F. King, Generalized CP and $\Delta\left(3 n^{2}\right)$ Family Symmetry for Semi-Direct Predictions of the PMNS Matrix, Phys. Rev. D 93 (2016) 025013 [arXiv:1510.03188] [INSPIRE].

[58] C.-C. Li, C.-Y. Yao and G.-J. Ding, Lepton Mixing Predictions from Infinite Group Series $D_{9 n, 3 n}^{(1)}$ with Generalized CP, JHEP 05 (2016) 007 [arXiv: 1601.06393] [INSPIRE].

[59] C.-Y. Yao and G.-J. Ding, CP Symmetry and Lepton Mixing from a Scan of Finite Discrete Groups, Phys. Rev. D 94 (2016) 073006 [arXiv:1606.05610] [InSPIRE].

[60] J.-N. Lu and G.-J. Ding, Alternative Schemes of Predicting Lepton Mixing Parameters from Discrete Flavor and CP Symmetry, Phys. Rev. D 95 (2017) 015012 [arXiv:1610.05682] [INSPIRE].

[61] S.-j. Rong, Lepton mixing patterns from the group $\Sigma(36 \times 3)$ with a generalized CP transformation, Phys. Rev. D 95 (2017) 076014 [arXiv: 1604.08482] [INSPIRE].

[62] J. Turner, Predictions for leptonic mixing angle correlations and nontrivial Dirac CP-violation from $A_{5}$ with generalized CP symmetry, Phys. Rev. D 92 (2015) 116007 [arXiv: 1507.06224] [INSPIRE].

[63] J.T. Penedo, S.T. Petcov and A.V. Titov, Neutrino mixing and leptonic CP-violation from $S_{4}$ flavour and generalised CP symmetries, JHEP 12 (2017) 022 [arXiv:1705.00309] [INSPIRE].

[64] P. Chen, G.-J. Ding, F. Gonzalez-Canales and J.W.F. Valle, Generalized $\mu-\tau$ reflection symmetry and leptonic CP-violation, Phys. Lett. B 753 (2016) 644 [arXiv:1512.01551] [INSPIRE]. 
[65] P. Chen, G.-J. Ding, F. Gonzalez-Canales and J.W.F. Valle, Classifying CP transformations according to their texture zeros: theory and implications, Phys. Rev. D 94 (2016) 033002 [arXiv: 1604.03510] [INSPIRE].

[66] P. Chen, G.-J. Ding and S.F. King, Leptogenesis and residual CP symmetry, JHEP 03 (2016) 206 [arXiv: 1602.03873] [INSPIRE].

[67] C. Hagedorn and E. Molinaro, Flavor and CP symmetries for leptogenesis and $0 \nu \beta \beta$ decay, Nucl. Phys. B 919 (2017) 404 [arXiv:1602.04206] [inSPIRE].

[68] C.-C. Li and G.-J. Ding, Implications of residual CP symmetry for leptogenesis in a model with two right-handed neutrinos, Phys. Rev. D 96 (2017) 075005 [arXiv:1701.08508] [INSPIRE].

[69] A. Bovier, M. Luling and D. Wyler, Finite Subgroups of SU(3), J. Math. Phys. 22 (1981) 1543 [INSPIRE].

[70] J.A. Escobar and C. Luhn, The Flavor Group $\Delta\left(6 n^{2}\right)$, J. Math. Phys. 50 (2009) 013524 [arXiv: 0809.0639] [INSPIRE].

[71] C. Jarlskog, Commutator of the Quark Mass Matrices in the Standard Electroweak Model and a Measure of Maximal CP-violation, Phys. Rev. Lett. 55 (1985) 1039 [INSPIRE].

[72] G.C. Branco, R.G. Felipe and F.R. Joaquim, Leptonic CP-violation, Rev. Mod. Phys. 84 (2012) 515 [arXiv:1111.5332] [INSPIRE].

[73] G.C. Branco, L. Lavoura and M.N. Rebelo, Majorana Neutrinos and CP Violation in the Leptonic Sector, Phys. Lett. B 180 (1986) 264 [InSPIRE].

[74] J.F. Nieves and P.B. Pal, Minimal Rephasing Invariant CP Violating Parameters With Dirac and Majorana Fermions, Phys. Rev. D 36 (1987) 315 [InSPIRE].

[75] J.F. Nieves and P.B. Pal, Rephasing invariant CP-violating parameters with Majorana neutrinos, Phys. Rev. D 64 (2001) 076005 [hep-ph/0105305] [INSPIRE].

[76] DUNE collaboration, R. Acciarri et al., Long-Baseline Neutrino Facility (LBNF) and Deep Underground Neutrino Experiment (DUNE). Volume 1: The LBNF and DUNE Projects, arXiv: 1601.05471 [INSPIRE].

[77] DUNE collaboration, R. Acciarri et al., Long-Baseline Neutrino Facility (LBNF) and Deep Underground Neutrino Experiment (DUNE). Volume 2: The Physics Program for DUNE at $L B N F$, arXiv: 1512.06148 [INSPIRE].

[78] DUNE collaboration, J. Strait et al., Long-Baseline Neutrino Facility (LBNF) and Deep Underground Neutrino Experiment (DUNE). Volume 3: Long-Baseline Neutrino Facility for DUNE June 24, 2015, arXiv:1601.05823 [INSPIRE].

[79] DUNE collaboration, R. Acciarri et al., Long-Baseline Neutrino Facility (LBNF) and Deep Underground Neutrino Experiment (DUNE). Volume 4: The DUNE Detectors at LBNF, arXiv: 1601.02984 [INSPIRE].

[80] Hyper-Kamiokande Working Group, E. Kearns et al., Hyper-Kamiokande Physics Opportunities, arXiv:1309.0184 [INSPIRE].

[81] Hyper-Kamiokande Working Group, K. Abe et al., A Long Baseline Neutrino Oscillation Experiment Using J-PARC Neutrino Beam and Hyper-Kamiokande, arXiv:1412.4673 [INSPIRE]. 
[82] Hyper-Kamiokande Proto- collaboration, K. Abe et al., Physics Potentials with the Second Hyper-Kamiokande Detector in Korea, arXiv:1611.06118 [INSPIRE].

[83] E. Baussan, M. Dracos, T. Ekelof, E.F. Martinez, H. Ohman and N. Vassilopoulos, The use the a high intensity neutrino beam from the ESS proton linac for measurement of neutrino CP-violation and mass hierarchy, arXiv:1212.5048 [INSPIRE].

[84] ESSnuSB collaboration, E. Baussan et al., A very intense neutrino super beam experiment for leptonic CP-violation discovery based on the European spallation source linac, Nucl. Phys. B 885 (2014) 127 [arXiv: 1309.7022] [INSPIRE].

[85] EXO-200 collaboration, M. Auger et al., Search for Neutrinoless Double-Beta Decay in ${ }^{136}$ Xe with EXO-200, Phys. Rev. Lett. 109 (2012) 032505 [arXiv:1205.5608] [INSPIRE].

[86] EXO-200 collaboration, J.B. Albert et al., Search for Majorana neutrinos with the first two years of EXO-200 data, Nature $\mathbf{5 1 0}$ (2014) 229 [arXiv:1402.6956] [INSPIRE].

[87] KamLAND-Zen collaboration, A. Gando et al., Limit on Neutrinoless $\beta \beta$ Decay of ${ }^{136}$ Xe from the First Phase of KamLAND-Zen and Comparison with the Positive Claim in ${ }^{76} \mathrm{Ge}$, Phys. Rev. Lett. 110 (2013) 062502 [arXiv:1211.3863] [InSPIRE].

[88] Planck collaboration, P.A.R. Ade et al., Planck 2013 results. XVI. Cosmological parameters, Astron. Astrophys. 571 (2014) A16 [arXiv:1303.5076] [INSPIRE].

[89] P. Ballett, S.F. King, C. Luhn, S. Pascoli and M.A. Schmidt, Testing atmospheric mixing sum rules at precision neutrino facilities, Phys. Rev. D 89 (2014) 016016 [arXiv: 1308.4314] [INSPIRE].

[90] P. Ballett, S.F. King, C. Luhn, S. Pascoli and M.A. Schmidt, Testing solar lepton mixing sum rules in neutrino oscillation experiments, JHEP 12 (2014) 122 [arXiv:1410.7573] [INSPIRE].

[91] S.T. Petcov, Predicting the values of the leptonic CP-violation phases in theories with discrete flavour symmetries, Nucl. Phys. B 892 (2015) 400 [arXiv:1405.6006] [INSPIRE].

[92] I. Girardi, S.T. Petcov and A.V. Titov, Determining the Dirac CP-violation Phase in the Neutrino Mixing Matrix from Sum Rules, Nucl. Phys. B 894 (2015) 733 [arXiv: 1410.8056] [INSPIRE].

[93] I. Girardi, S.T. Petcov and A.V. Titov, Predictions for the Leptonic Dirac CP-violation Phase: a Systematic Phenomenological Analysis, Eur. Phys. J. C 75 (2015) 345 [arXiv: 1504.00658] [INSPIRE].

[94] I. Girardi, S.T. Petcov and A.V. Titov, Predictions for the Majorana CP-violation Phases in the Neutrino Mixing Matrix and Neutrinoless Double Beta Decay, Nucl. Phys. B 911 (2016) 754 [arXiv: 1605.04172] [INSPIRE].

[95] JUNO collaboration, F. An et al., Neutrino Physics with JUNO, J. Phys. G 43 (2016) 030401 [arXiv: 1507.05613] [INSPIRE].

[96] S.-B. Kim, New results from RENO and prospects with RENO-50, talk given at the Neutrino Oscillation Workshop (NOW 2014), Conca Specchiulla, Otranto, Lecce, Italy, 7-14 September 2014, Nucl. Part. Phys. Proc. 265-266 (2015) 93 [arXiv:1412.2199] [InSPIRE].

[97] S. Geer, Neutrino beams from muon storage rings: Characteristics and physics potential, Phys. Rev. D 57 (1998) 6989 [Erratum ibid. D 59 (1999) 039903] [hep-ph/9712290] [INSPIRE]. 
[98] A. De Rujula, M.B. Gavela and P. Hernández, Neutrino oscillation physics with a neutrino factory, Nucl. Phys. B 547 (1999) 21 [hep-ph/9811390] [INSPIRE].

[99] ISS PHYsics Working Group, A. Bandyopadhyay, Physics at a future Neutrino Factory and super-beam facility, Rept. Prog. Phys. 72 (2009) 106201 [arXiv:0710.4947] [InSPIRE].

[100] UTFiT collaboration, M. Bona et al., The 2004 UTfit collaboration report on the status of the unitarity triangle in the standard model, JHEP 07 (2005) 028 [hep-ph/0501199] [INSPIRE].

[101] UTfiT collaboration, M. Bona et al., Model-independent constraints on $\Delta F=2$ operators and the scale of new physics, JHEP 03 (2008) 049 [arXiv:0707.0636] [INSPIRE].

[102] C.D. Froggatt and H.B. Nielsen, Hierarchy of Quark Masses, Cabibbo Angles and CP-violation, Nucl. Phys. B 147 (1979) 277 [INSPIRE].

[103] S. Antusch, S.F. King, C. Luhn and M. Spinrath, Right Unitarity Triangles and Tri-Bimaximal Mixing from Discrete Symmetries and Unification, Nucl. Phys. B 850 (2011) 477 [arXiv: 1103.5930] [INSPIRE].

[104] S. Antusch, M. Holthausen, M.A. Schmidt and M. Spinrath, Solving the Strong CP Problem with Discrete Symmetries and the Right Unitarity Triangle, Nucl. Phys. B 877 (2013) 752 [arXiv:1307.0710] [INSPIRE]. 